\title{
Health Education Materials for the Workplace: Tools
}

Ulrich Madeja

David Wofford

Carolyn Rodehau

Follow this and additional works at: https://knowledgecommons.popcouncil.org/departments_sbsr-rh How does access to this work benefit you? Let us know!

\section{Recommended Citation}

Madeja, Ulrich, David Wofford, and Carolyn Rodehau. 2017. "Health Education Materials for the Workplace: Tools." Evidence Project/Meridian in partnership with Bayer. 

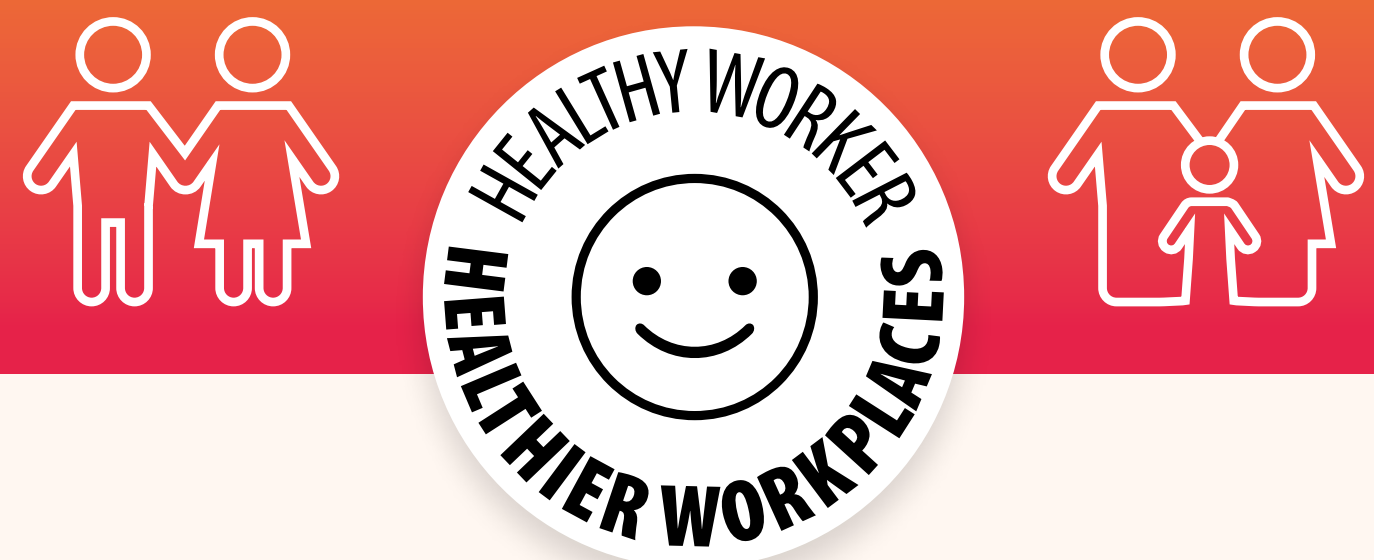

Do you know the Facts about family planning?

Check your knowledge:

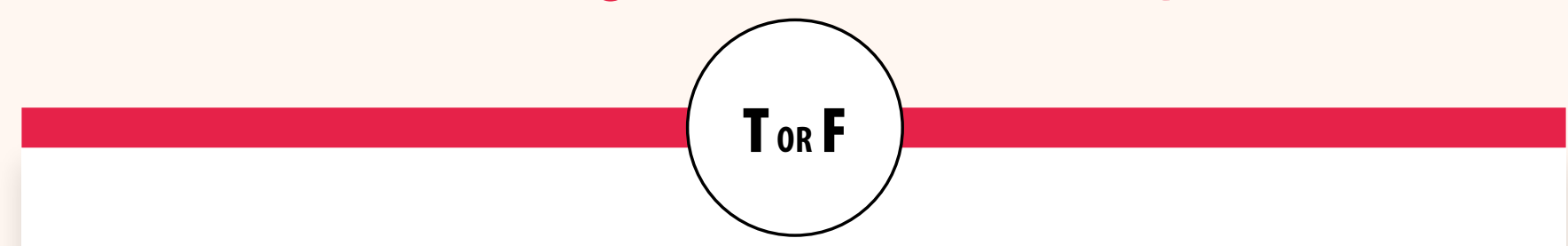

(1) IT IS BEST FOR YOUR HEALTH TO WAIT

2 YEARS BEFORE TRYING TO HAVE

(7) INJECTABLES MAY CAUSE TEMPORARY ANOTHER CHILD AFTER GIVING BIRTH.

CHANGES IN MENSTRUAL BLEEDING.

(2) A MENSTRUATING WOMAN CANNOT GET PREGNANT.

8 THE PILL CAN BE TAKEN RIGHT AFTER YOU'VE HAD RELATIONS (SEX) AND PREVENT YOU FROM GETTING PREGNANT.

3) CONDOMS ARE THE ONLY CONTRACEPTIVE METHOD THAT PREVENTS BOTH PREGNANCY AND SEXUALLY TRANSMITTED INFECTIONS (STIs).

9 ONLY BREASTFEEDING A CHILD DURING THE FIRST 6 MONTHS HELPS TO AVOID PREGNANCY.

(4) THE PILl CAN CAUSE INFERTILITY.

5 THE IUD COIL CAN MOVE THROUGH THE WOMB AND PIERCE OTHER ORGANS.

True or False? ANSWERS:

(6) THE PILLS CAUSE BIRTH DEFECTS.

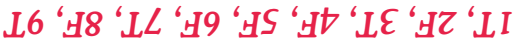

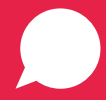

Talk to a health care provider you trust at your workplace or in your community 

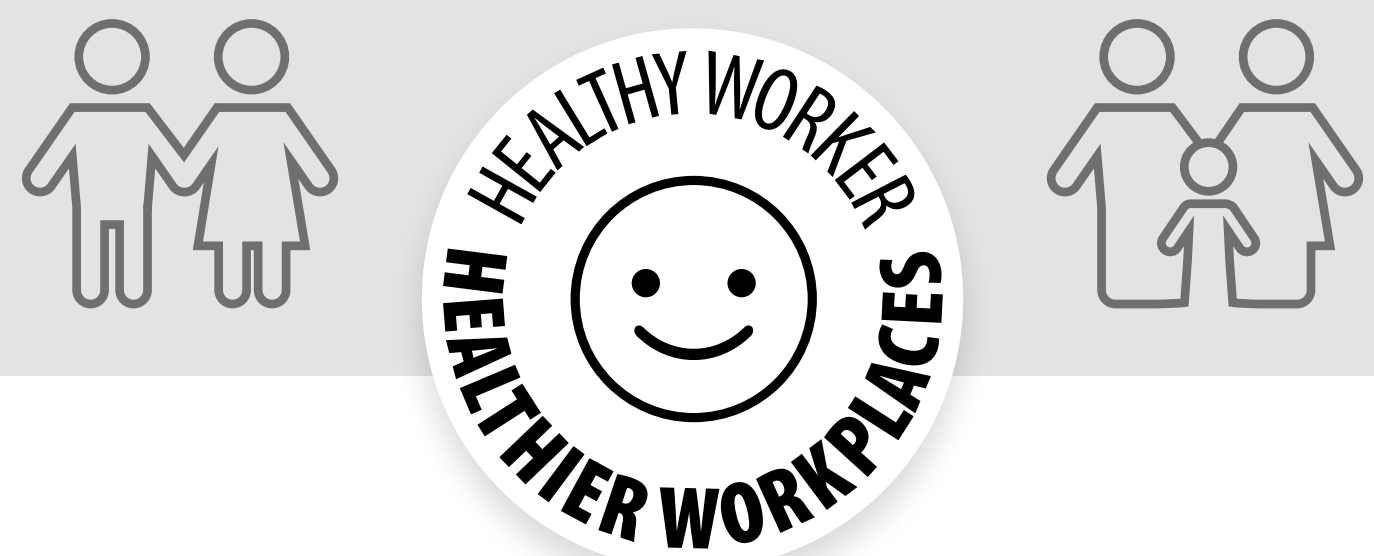

Do you know the Facts about family planning?

Check your knowledge:

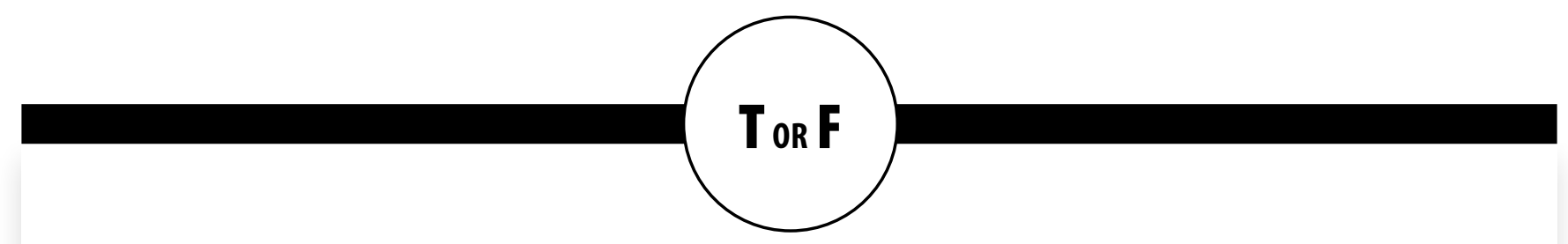

(1) IT IS BEST FOR YOUR HEALTH TO WAIT 2 YEARS BEFORE TRYING TO HAVE

7 INJECTABLES MAY CAUSE TEMPORARY ANOTHER CHILD AFTER GIVING BIRTH. CHANGES IN MENSTRUAL BLEEDING.

(2) A MENSTRUATING WOMAN CANNOT GEt PREGNANT.

8 THE PILL CAN BE TAKEN RIGHT AFTER YOU'VE HAD RELATIONS (SEX) AND PREVENT YOU FROM GETTING PREGNANT.

3) CONDOMS ARE THE ONLY CONTRACEPTIVE METHOD THAT PREVENTS BOTH PREGNANCY AND SEXUALLY TRANSMITTED INFECTIONS (STIs).

9 ONLY BREASTFEEDING A CHILD DURING THE FIRST 6 MONTHS HELPS TO AVOID PREGNANCY.

(4) THE PILL CAN CAUSE INFERTILITY.

5 THE IUD COIL CAN MOVE THROUGH THE WOMB AND PIERCE OTHER ORGANS.

True or False? ANSWERS:

6 THE PILLS CAUSE BIRTH DEFECTS.

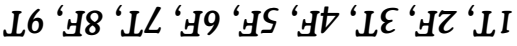

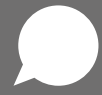

Talk to a health care provider you trust at your workplace or in your community 


\section{Which family planning method is best for you?}

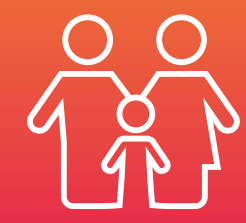

\section{$99 \%$}

SUPEREFFECTIVE

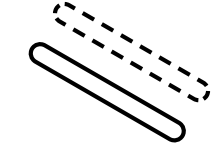

THE IMPLANT

3-5 Years

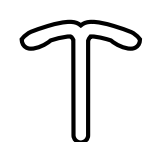

IUS

3-5 Years

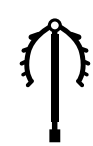

IUD

5-10 Years

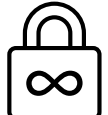

STERILIZATION

Forever

\section{HIGHLY EFFECTIVE}

$91-94 \%$

*At Typical Use

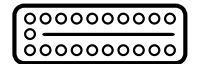

THE PILL

Every Day

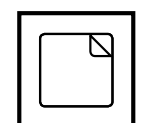

THE PATCH

Every Week

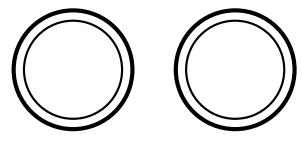

VAGINAL RING

Every Month

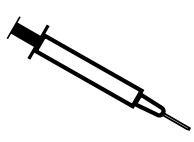

INJECTION

1-3 Months

\section{LESS EFFECTIVE}

$72-82 \%$

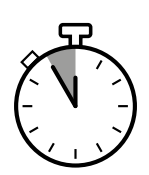

WITHDRAWAL

Every Time

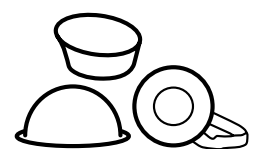

CERVICAL CAP, DIAPHRAGM \& SPONGE

EveryTime

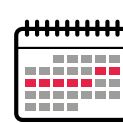

STANDARD DAYS

METHOD

Every Day

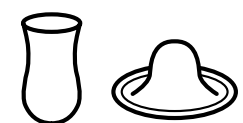

SPERMICIDES

Every Time

FEMALE \& MALE CONDOM

Single Use 


\section{(i) method is best for you?

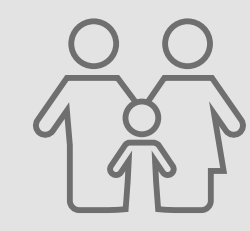

\section{SUPER EFFECTIVE \\ $99 \%$}

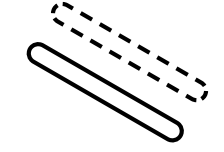

THE IMPLANT

3-5 Years

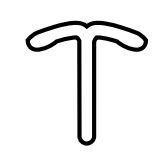

IUS

3-5 Years

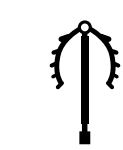

IUD

5-10 Years

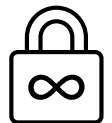

STERILIZATION

Forever

\section{HIGHLY EFFECTIVE}

$91-94 \%$

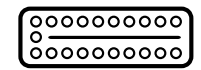

THE PILL

Every Day

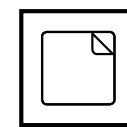

THE PATCH

Every Week

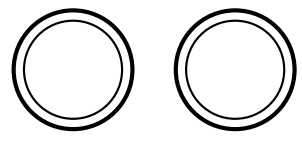

VAGINAL RING

Every Month

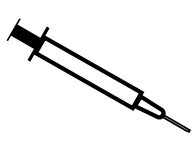

INJECTION

1-3 Months

\section{LESS EFFECTIVE}

$72-82 \%$

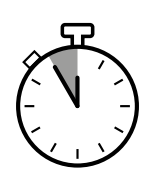

WITHDRAWAL

Every Time

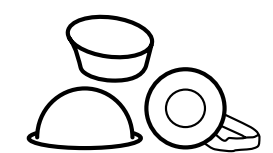

CERVICAL CAP, DIAPHRAGM \& SPONGE EveryTime

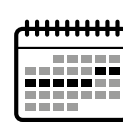

STANDARD DAYS

METHOD

Every Day

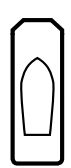

SPERMICIDES

Every Time

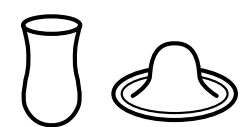

FEMALE \& MALE CONDOM 
(1) SPACING YOUR CHILDREN IS THE BEST WAY TO ENSURE A HEALTHY BABY, MOTHER AND FAMILY. This spacing allows mothers to recover from birth, parents to have enough money and time for their children. To space your children, use modern family planning and wait at least 2 years after giving birth before trying to get pregnant again.

(!) YOU CAN GET PREGNANT DURING MENSTRUATION. If you are using $a$ natural family planning (standard days method) you must carefully track your cycle and not assume that you cannot get pregnant.

(!) CONDOMS ARE THE ONLY FAMILY PLANNING METHOD THAT PREVENTS BOTH PREGNANCY AND SEXUALLY TRANSMITTED DISEASES, INCLUDING HIV. Nothing can pass through $a$ condom as long as it is not ripped.

\section{(!) CONTRACEPTIVES do nOt CAUSE} INFERTILITY. You may find it takes a few months for your body to return to a state where you can become pregnant again. This is only temporary and not permanent. Fertility returns for a healthy women no matter how long she has taken a hormonal family planning method.

(!) THE IUD IS A HIGHLY AND EFFECTIVE METHOD THAT CAN PREVENT PREGNANCY FOR UP TO 5 YEARS FOR THE IUS AND UP TO 10 YEARS FOR THE COPPER IUD. When inserted correctly by $a$ trained person, it stays in place. It can never pass from the uterus to the other parts of the body.

(!) MODERN CONTRACEPTIVES ARE WELL TOLERATED AND HIGHLY RELIABLE.

They may even protect you from some forms of cancer, such as those of the ovary and the uterus.
Implants may protect against pelvic inflammatory disease. Only women over the age of 40 who smoke may be advised to avoid the combine oral contraceptive pill.

(!) THE PILL WILL NOT CAUSE BIRTH DEFECTS or harm the fetus if you become pregnant while taking the pill or accidentally start to take it when you are already pregnant.

(!) INJECTABLES ARE A HIGHLY EFFECTIVE SHORT-TERM METHOD THAT REQUIRES A SHOT ONCE A MONTH OR EVERY 3 MONTHS. You may experience irregular bleeding, heavy bleeding, or prolonged bleeding for a very short time. A few women sometime have headaches, dizziness, breast tenderness, and weight gain. These are not signs of illness. These problems almost always go away within the first few months of using the injectable.
(!) THE PILL MUST BE TAKEN CONSISTENTLY EACH DAY IN ORDER TO PREVENT PREGNANCY. You cannot take the pill after having sex and be sure you are protected. If you miss a day or start your pack five days after the start of your menstrual period, then you must be sure to use a backup method for at least the next seven days. There are Emergency Contraceptive pills. These are stronger than your daily pill and should be used rarely and only during emergencies.

(!) BREASTFEEDING PREVENTS PREGNANCY UP TO SIX MONTHS after the child is born if used exclusively and no other foods and menstrual periods have not resumed. Women can also use progestin-only pills during breast-feeding. 
(?) SPACING YOUR CHILDREN IS THE BEST WAY TO ENSURE A HEALTHY BABY, MOTHER AND FAMILY. This spacing allows mothers to recover from birth, parents to have enough money and time for their children. To space your children, use modern family planning and wait at least 2 years after giving birth before trying to get pregnant again.

(!) YOU CAN GET PREGNANT DURING MENSTRUATION. If you are using $a$ natural family planning (standard days method) you must carefully track your cycle and not assume that you cannot get pregnant.

(!) CONDOMS ARE THE ONLY FAMILY PLANNING METHOD THAT PREVENTS BOTH PREGNANCY AND SEXUALLY TRANSMITTED DISEASES, INCLUDING HIV. Nothing can pass through $a$ condom as long as it is not ripped.

\section{(!) CONTRACEPTIVES Do NOt CAUSE} INFERTILITY. You may find it takes a few months for your body to return to a state where you can become pregnant again. This is only temporary and not permanent. Fertility returns for a healthy women no matter how long she has taken a hormonal family planning method.

(!) THE IUD IS A HIGHLY AND EFFECTIVE METHOD THAT CAN PREVENT PREGNANCY FOR UP TO 5 YEARS FOR THE IUS AND UP TO 10 YEARS FOR THE COPPER IUD. When inserted correctly by $a$ trained person, it stays in place. It can never pass from the uterus to the other parts of the body.

(!) MODERN CONTRACEPTIVES ARE WELL TOLERATED AND HIGHLY RELIABLE.

They may even protect you from some forms of cancer, such as those of the ovary and the uterus.
Implants may protect against pelvic inflammatory disease. Only women over the age of 40 who smoke may be advised to avoid the combine oral contraceptive pill.

(!) THE PILL WILL NOT CAUSE BIRTH DEFECTS or harm the fetus if you become pregnant while taking the pill or accidentally start to take it when you are already pregnant.

(!) INJECTABLES ARE A HIGHLY EFFECTIVE SHORT-TERM METHOD THAT REQUIRES A SHOT ONCE A MONTH OR EVERY 3 MONTHS. You may experience irregular bleeding, heavy bleeding, or prolonged bleeding for a very short time. A few women sometime have headaches, dizziness, breast tenderness, and weight gain. These are not signs of illness. These problems almost always go away within the first few months of using the injectable.
(!) THE PILL MUST BE TAKEN CONSISTENTLY EACH DAY IN ORDER TO PREVENT PREGNANCY. You cannot take the pill after having sex and be sure you are protected. If you miss a day or start your pack five days after the start of your menstrual period, then you must be sure to use a backup method for at least the next seven days. There are Emergency Contraceptive pills. These are stronger than your daily pill and should be used rarely and only during emergencies.

(!) BREASTFEEDING PREVENTS PREGNANCY UP TO SIX MONTHS after the child is born if used exclusively and no other foods and menstrual periods have not resumed. Women can also use progestin-only pills during breast-feeding. 

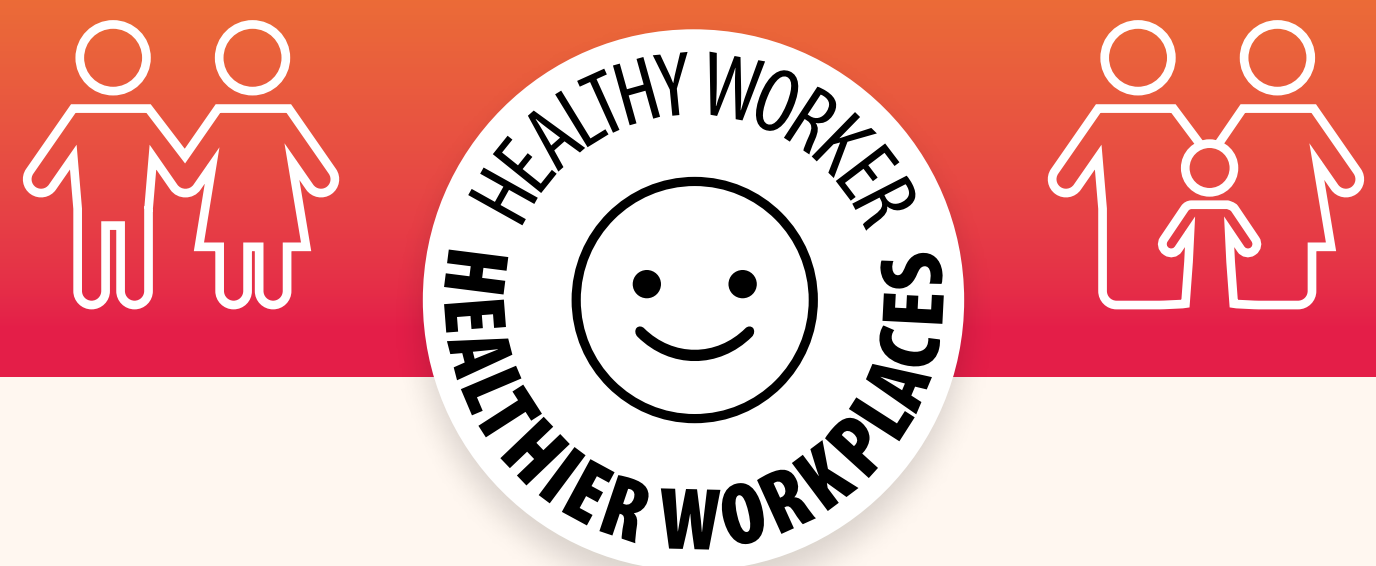

\section{Family planning: \\ By choice not chance!}

\section{?}

What is the MOST EFFECTIVE way to

- PLAN YOUR FAMILY

- PREVENT UNWANTEd PREGNANCIES

- AVOID UNSAFE ABORTIONS

\section{!}

Women workers use MODERN hormonal family planning!

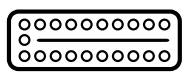

PILL

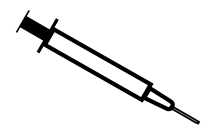

INJECTABLE

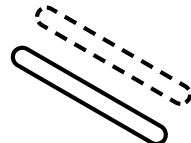

IMPLANT

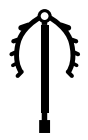

IUD

Talk to a health care provider you trust at your workplace or in your community 

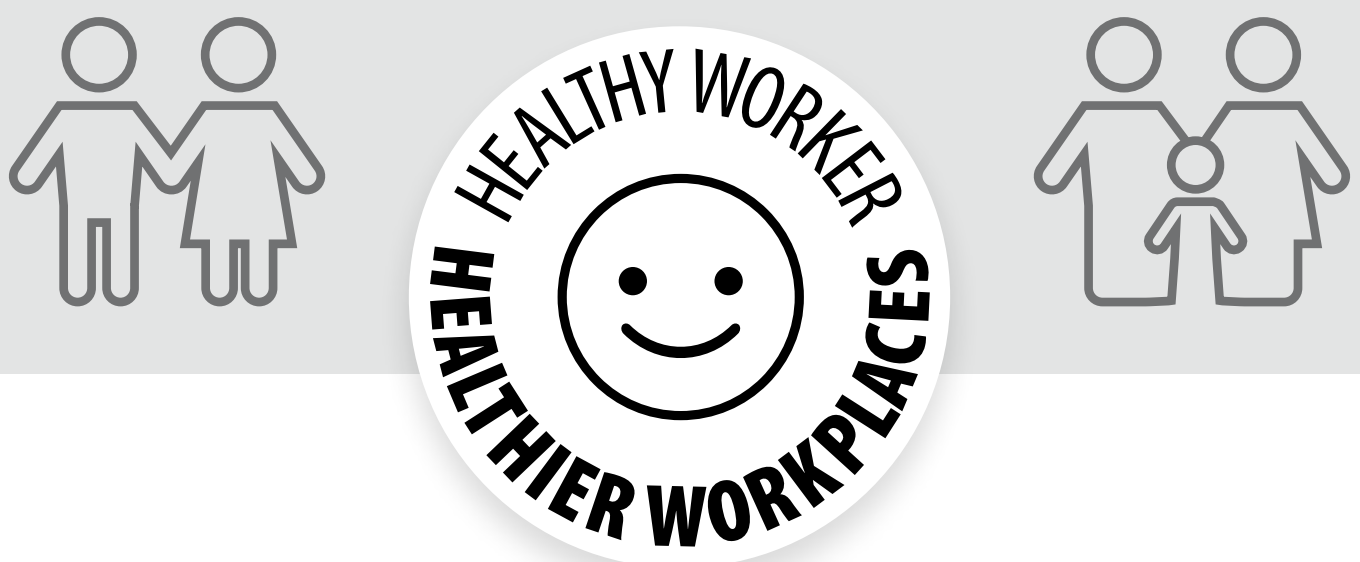

Family planning:

By choice not chance!

\section{?}

What is the MOST EFFECTIVE way to

- PLAN YOUR FAMILY

- PREVENT UNWANTEd PREGNANCIES

- AVOID UNSAFE ABORTIONS

\section{!}

Women workers use MODERN hormonal family planning!

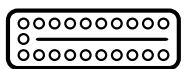

PILL

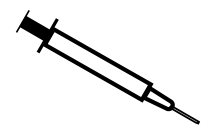

INJECTABLE

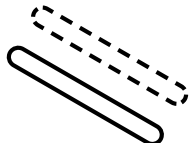

IMPLANT

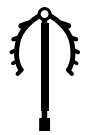

IUD

Talk to a health care provider you trust at your workplace or in your community 

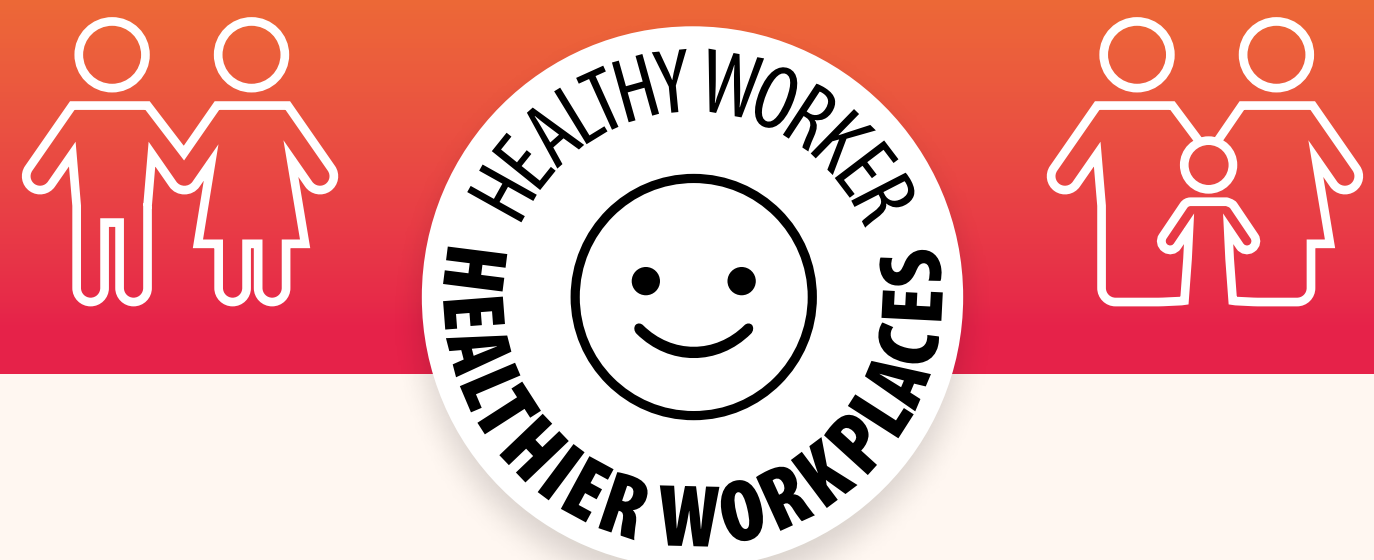

\section{Have a healthier family with more time and money for your children!}

\section{HOW?}

Wait until your child turns two to become pregnant again.

(Vour babies GROW UP bigger, stronger, healthier

(Vou will be HEALTHIER AND STRONGER to carry your new baby
(1) YOUR NEWBORNS are less likely to die, be born too early, or be under-weight

(V) You will have MORE INCOME to provide for THE FUTURE of your child

Which family planning method is right for you?

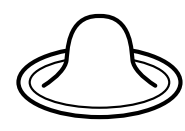

CONDOM

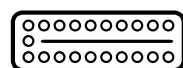

PILL

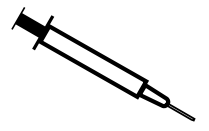

INJECTABLE IMPLANT

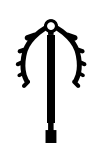

IUD

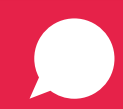

Talk to a health care provider you trust at your workplace or in your community 

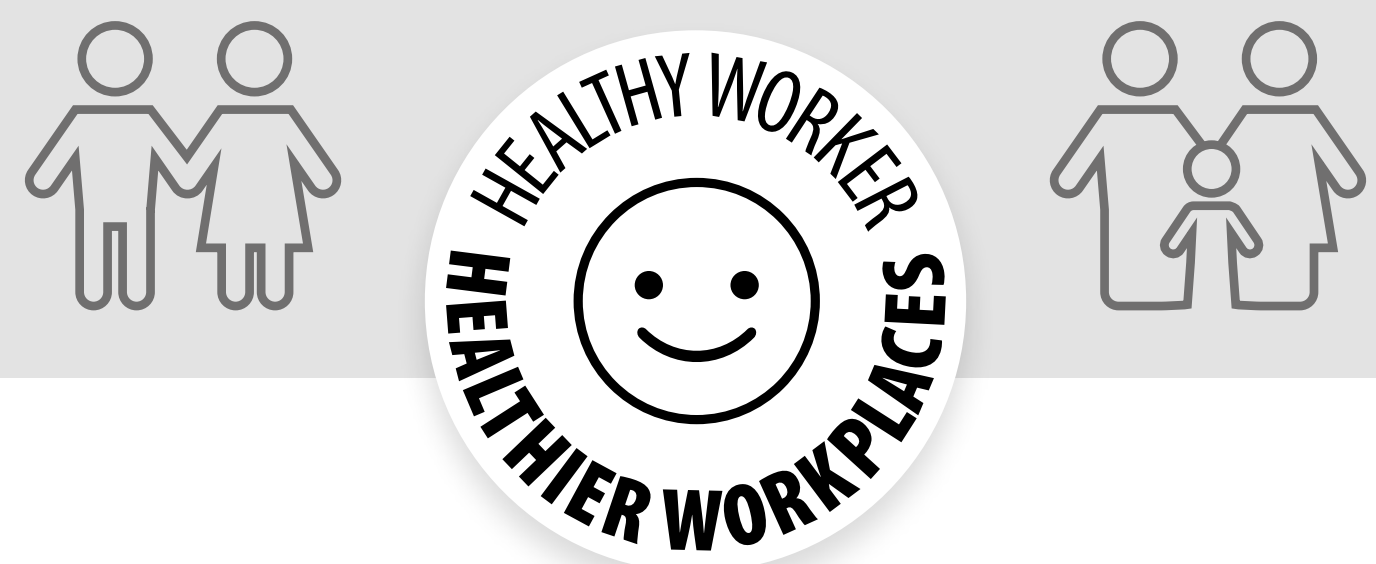

Have a healthier family with more time and money for your children!

\section{HOW?}

Wait until your child turns two to become pregnant again.

(V) Your babies GROW UP bigger, stronger, healthier

(Vou will be HEALTHIER AND STRONGER to carry your new baby
(V) YOUR NEWBORNS are less likely to die, be born too early, or be under-weight

(V) You will have MORE INCOME to provide for THE FUTURE of your child

Which family planning method is right for you?

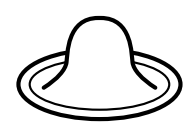

CONDOM

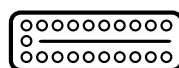

PILL

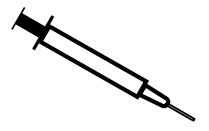

INJECTABLE IMPLANT

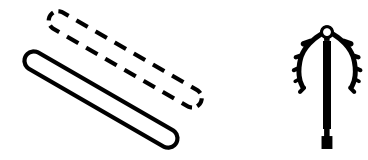

IUD

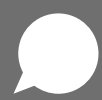

Talk to a health care provider you trust at your workplace or in your community 

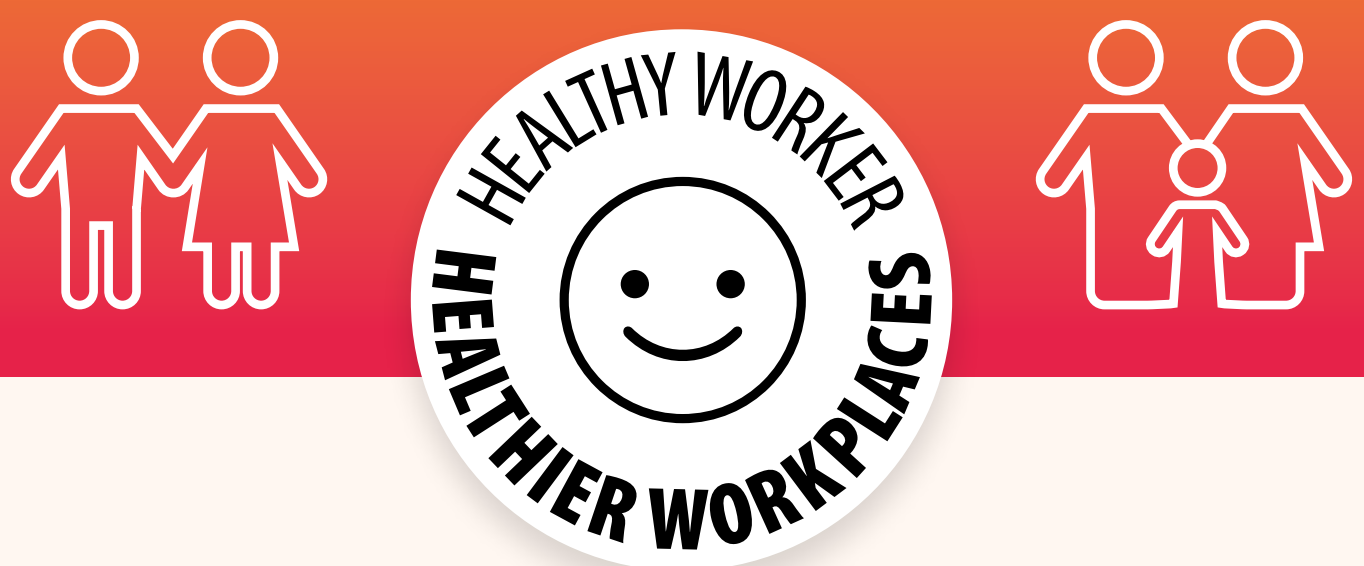

\section{Healthy workers space their children!}

\section{$?$}

How to have a HEALTHY BABY and $a$ HEALTHY FAMILY?

\section{!}

— WAIT UNTIL YOUR CHILD TURNS TWO TO BECOME PREGNANT AGAIN.

— USE A MODERN FAMILY PLANNING METHOD OF YOUR CHOICE:

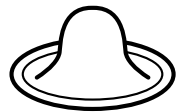

CONDOM

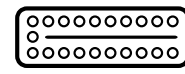

PILL

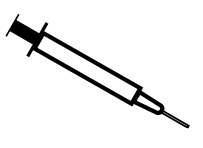

INJECTABLE

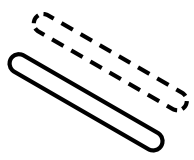

IMPLANT

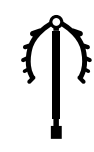

IUD

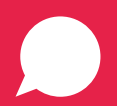

Talk to a health care provider you trust at your workplace or in your community 

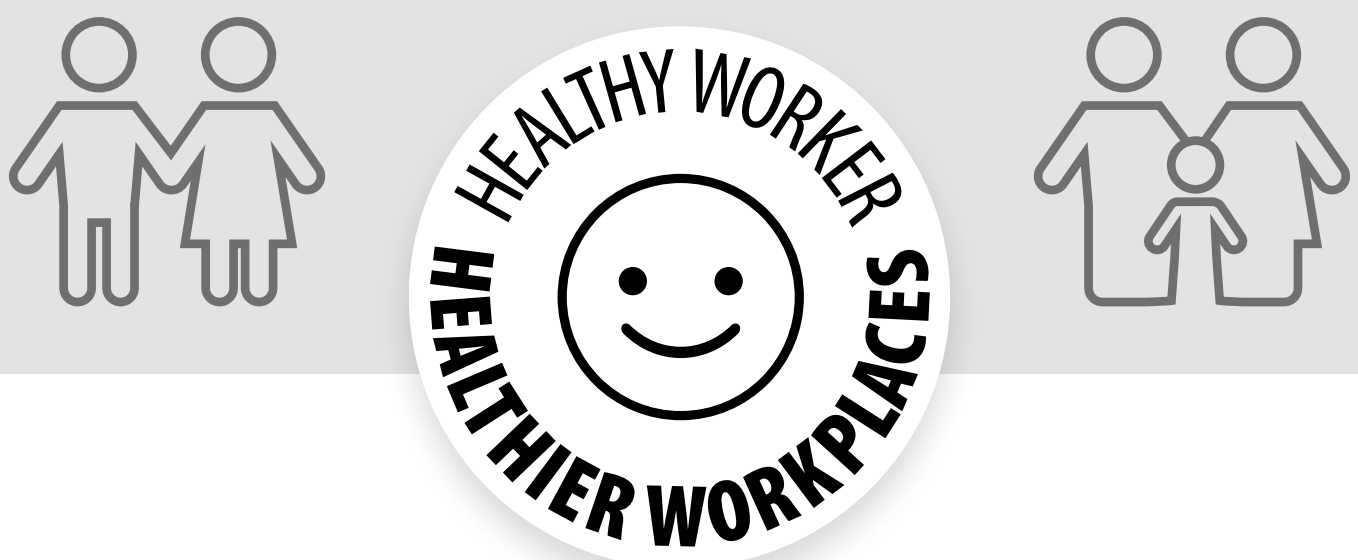

\section{Healthy workers space their children!}

\section{?}

How to have a HEALTHY BABY and $a$ HEALTHY FAMILY?

\section{!}

— WAIT UNTIL YOUR CHILD TURNS TWO TO BECOME PREGNANT AGAIN.

- USE A MODERN FAMILY PLANNING METHOD OF YOUR CHOICE:

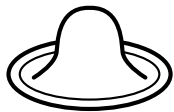

CONDOM

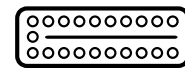

PILL

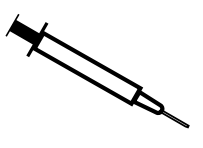

INJECTABLE

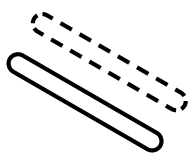

IMPLANT

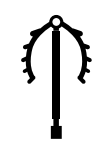

IUD

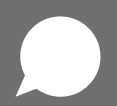

Talk to a health care provider you trust at your workplace or in your community 


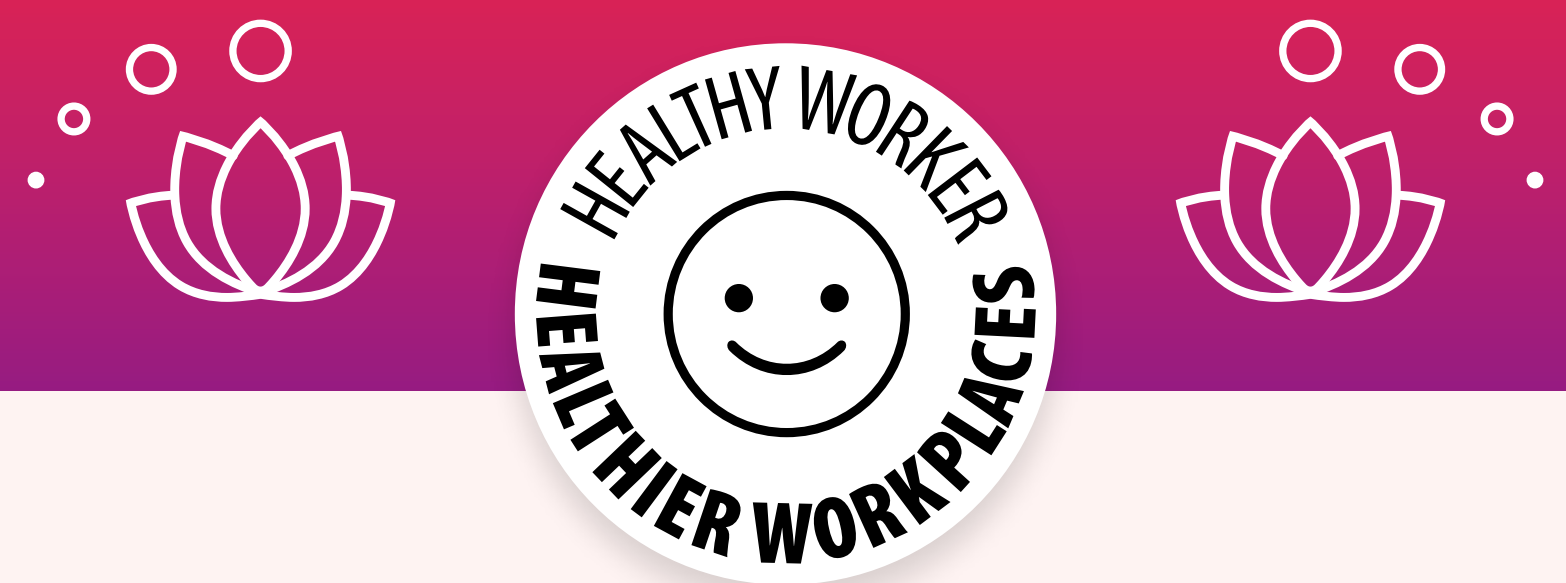

\section{Do you know the Facts about} menstrual hygiene and management? Check your knowledge:

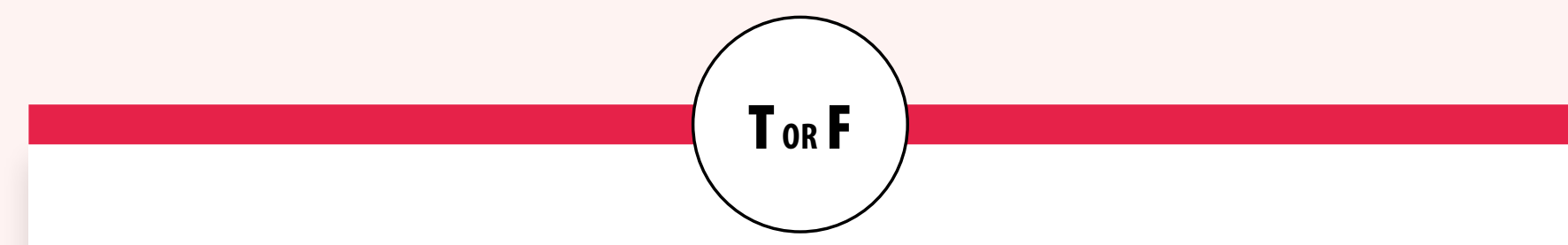

(1) MENSTRUATION HAPPENS FOR MOST WOMEN ABOUT ONCE A MONTH.

(2) STOMACH CRAMPS ARE THE PRIMARY SIGN OF MENSTRUATION.

(3) YOU SHOULD REFRAIN FROM ANY FORM OF EXERCISE DURING YOUR PERIOD.

(4) YOU SHOULD CHANGE YOUR CLOTH OR SANITARY PAD EVERY 2- 6 HOURS.

5) CARRYING A SUPPLY OF CLOTH OR SANITARY PADS WITH YOU IS GOOD PREPARATION FOR EMERGENCIES.

(6) PAIN RELIEVERS, A HOT BATH, OR A WARM COMPRESS CAN HELP EASE PAIN FROM CRAMPS.
7 THERE IS NO SENSE IN PREPARING FOR YOUR PERIOD - YOU NEVER KNOW WHEN IT WILL COME.

8 THE IDEA THAT CERTAIN FOODS SHOULD NOT BE EATEN AT THIS TIME IS A MYTH.

9 PADS CAN BE SAFELY DISPOSED OF IN THE GARBAGE PILE OR FLUSHED DOWN THE TOILET.

True or False? ANSWERS:

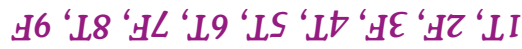

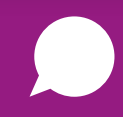

Talk to a health care provider you trust at your workplace or in your community 


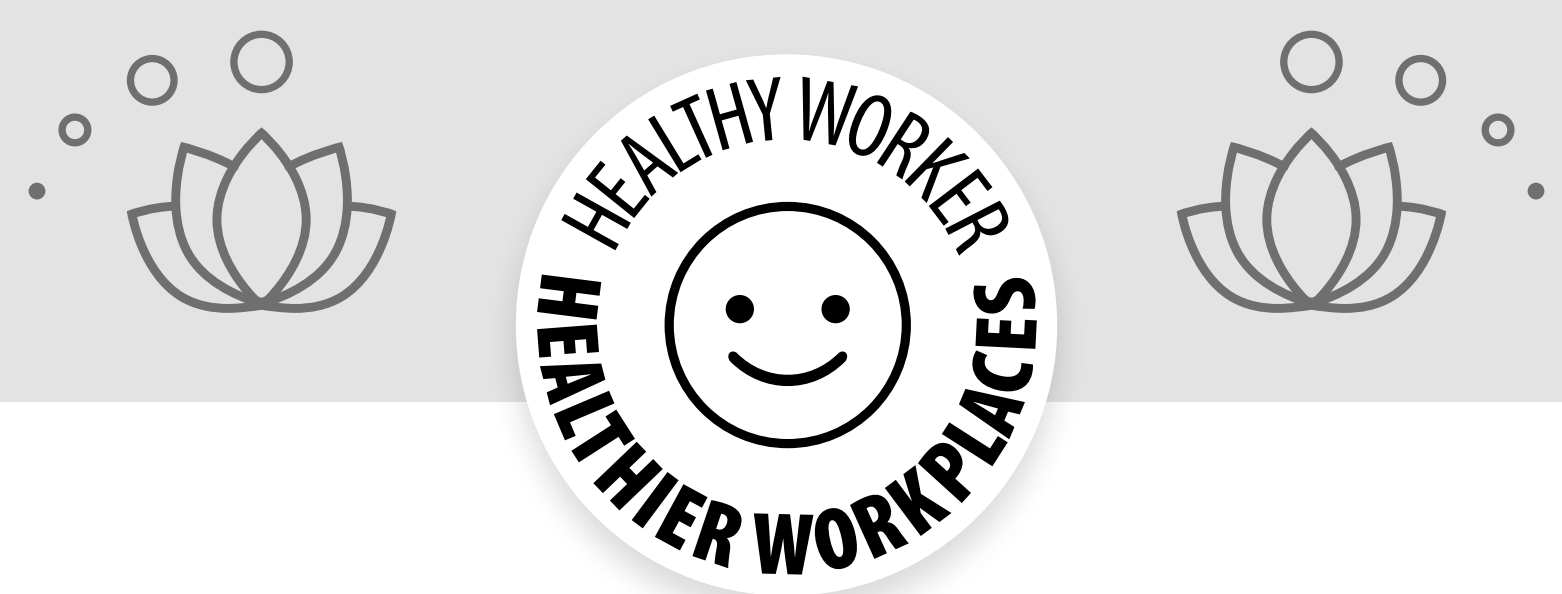

\section{Do you know the Facts about} menstrual hygiene and management? Check your knowledge:

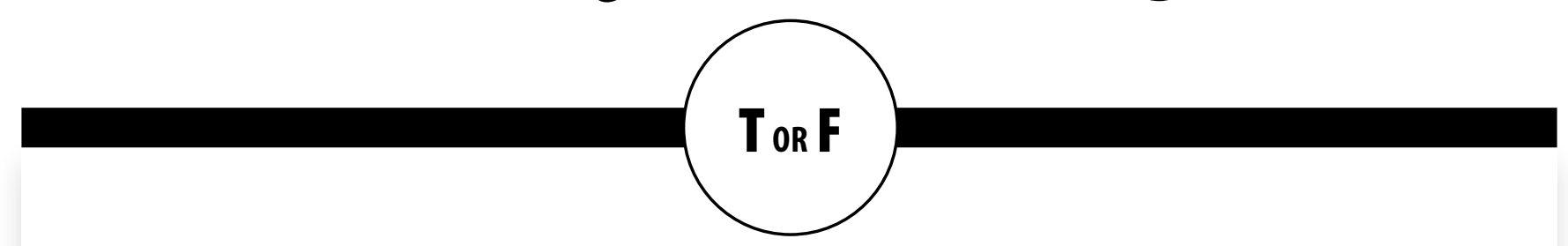

(1) MENSTRUATION HAPPENS FOR MOST WOMEN ABOUT ONCE A MONTH.

(2) STOMACH CRAMPS ARE THE PRIMARY SIGN OF MENSTRUATION.

(3) YOU SHOULD REFRAIN FROM ANY FORM OF EXERCISE DURING YOUR PERIOD.

(4) YOU SHOULD CHANGE YOUR CLOTH OR SANITARY PAD EVERY 2-6 HOURS.

5) CARRYING A SUPPLY OF CLOTH OR SANITARY PADS WITH YOU IS GOOD PREPARATION FOR EMERGENCIES.

6) PAIN RELIEVERS, A HOT BATH, OR A WARM COMPRESS CAN HELP EASE PAIN FROM CRAMPS.
7 THERE IS NO SENSE IN PREPARING FOR YOUR PERIOD - YOU NEVER KNOW WHEN IT WILL COME.

8 THE IDEA THAT CERTAIN FOODS SHOULD NOT BE EATEN AT THIS TIME IS A MYTH.

9 PADS CAN BE SAFELY DISPOSED OF IN THE GARBAGE PILE OR FLUSHED DOWN THE TOILET.

True or False? ANSWERS:

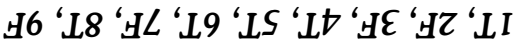

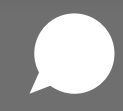

Talk to a health care provider you trust at your workplace or in your community 


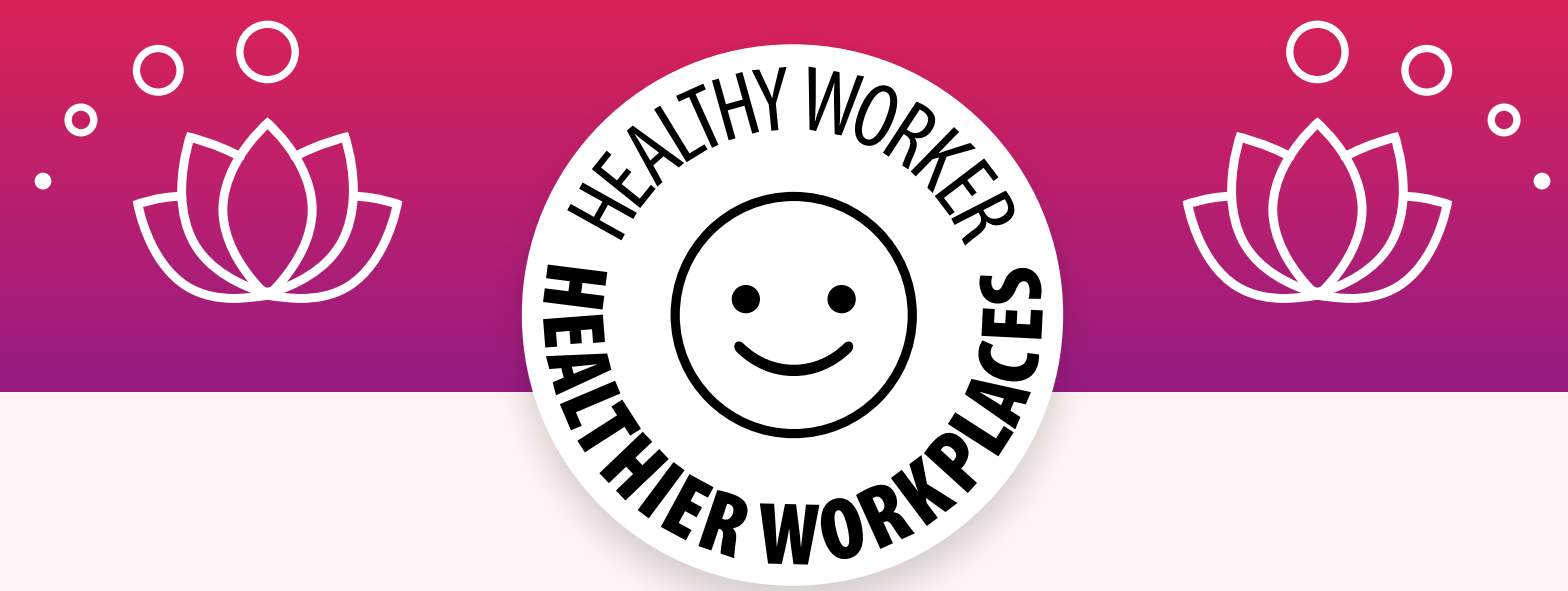

\section{Stay healthy during your period.}

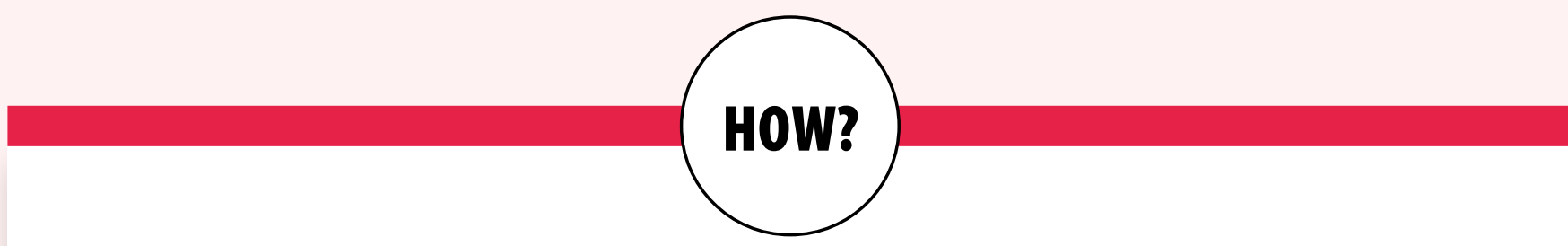

V StAY CLEAN. Bathe daily during your period.

V

USE A CLEAN CLOTH OR SANITARY PAD.

If using cloth, be sure it is washed thoroughly and hung in a sunny place to dry.

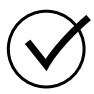

\section{CHANGE YOUR CLOTH OR SANITARY PAD} EVERY 2-6 HOURS. Women need to change their cloth or sanitary pad regularly to prevent infections.

\section{WEAR DARKER COLORED CLOTHING.}

During menstruation, you may bleed through your clothes. Consider wearing dark clothes on days when you're expecting your period or when your flow is at its heaviest in order to prepare and avoid embarrassment.
USE PAIN RELIEVERS, A HOT BATH, OR A WARM COMPRESS TO REDUCE PAIN.

Some women get cramps that are more severe than others. These remedies can help.

EXERCISE AND STRETCH. Both activities can also provide relief for cramps.

BE PREPARED. Your period may start at work. If you carry sanitary pads and $a$ pain reliever, then regular work activities can continue.

EAT RIGHT. It is a myth that certain foods should not be eaten during your period. You should eat iron rich foods to help with the blood loss and prevent anemia. Eating fruits or vegetables is also recommended. Try to avoid consuming caffeine and salt. 

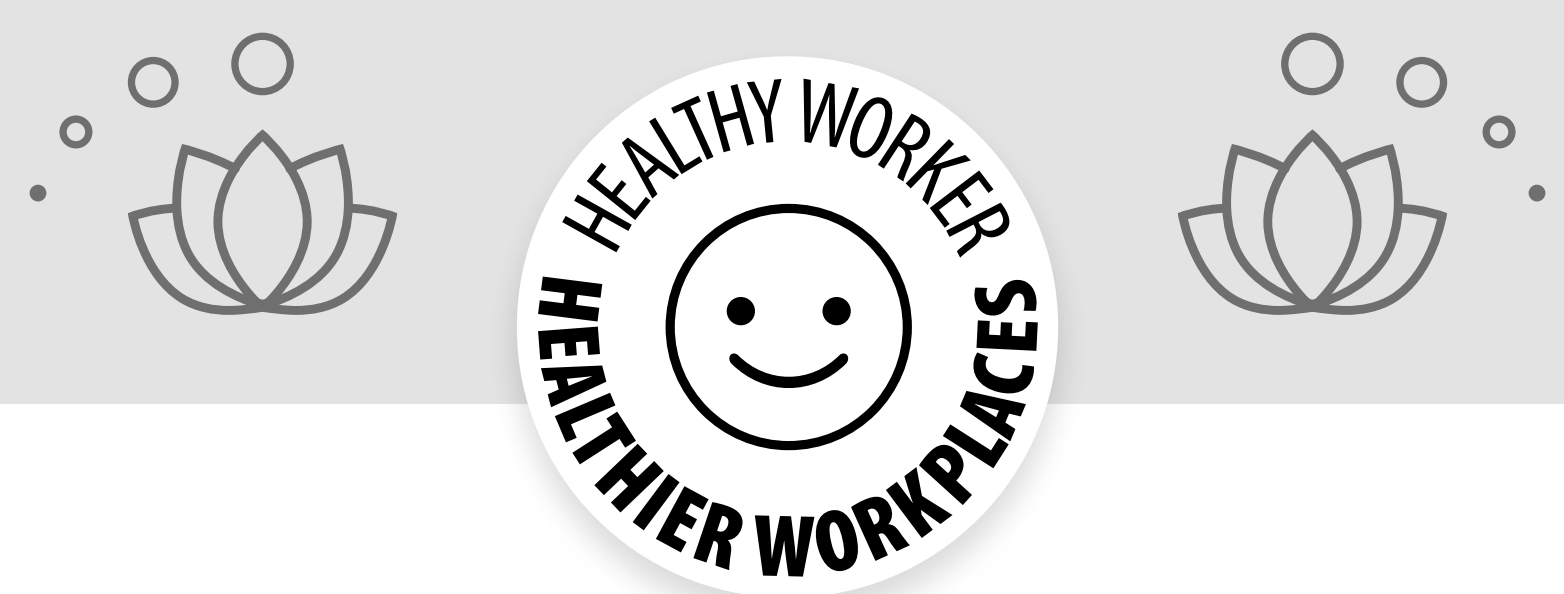

\section{Stay healthy during your period.}

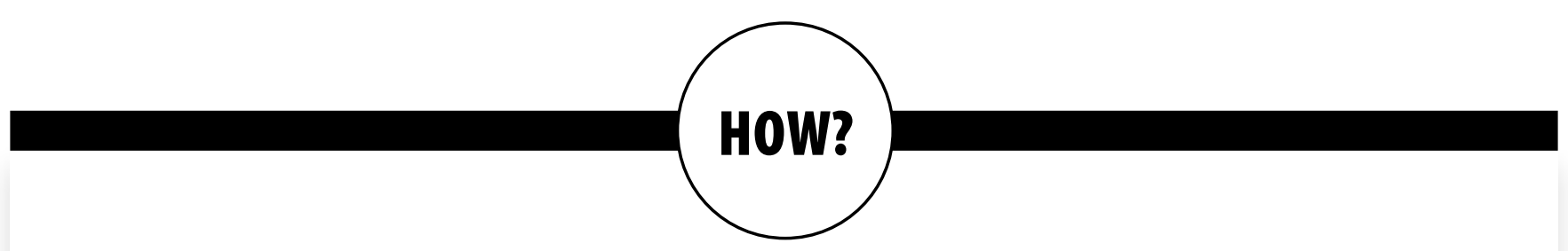

(V) StAY CLEAN. Bathe daily during your period.

V

USE A CLEAN CLOTH OR SANITARY PAD.

If using cloth, be sure it is washed thoroughly and hung in a sunny place to dry.

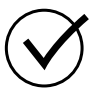

\section{CHANGE YOUR CLOTH OR SANITARY PAD}

EVERY 2-6 HOURS. Women need to change their cloth or sanitary pad regularly to prevent infections.

\section{WEAR DARKER COLORED CLOTHING.}

During menstruation, you may bleed through your clothes. Consider wearing dark clothes on days when you're expecting your period or when your flow is at its heaviest in order to prepare and avoid embarrassment.
USE PAIN RELIEVERS, A HOT BATH, OR A WARM COMPRESS TO REDUCE PAIN.

Some women get cramps that are more severe than others. These remedies can help.

EXERCISE AND STRETCH. Both activities can also provide relief for cramps.

BE PREPARED. Your period may start at work. If you carry sanitary pads and a pain reliever, then regular work activities can continue.

EAT RIGHT. It is a myth that certain foods should not be eaten during your period. You should eat iron rich foods to help with the blood loss and prevent anemia. Eating fruits or vegetables is also recommended. Try to avoid consuming caffeine and salt. 


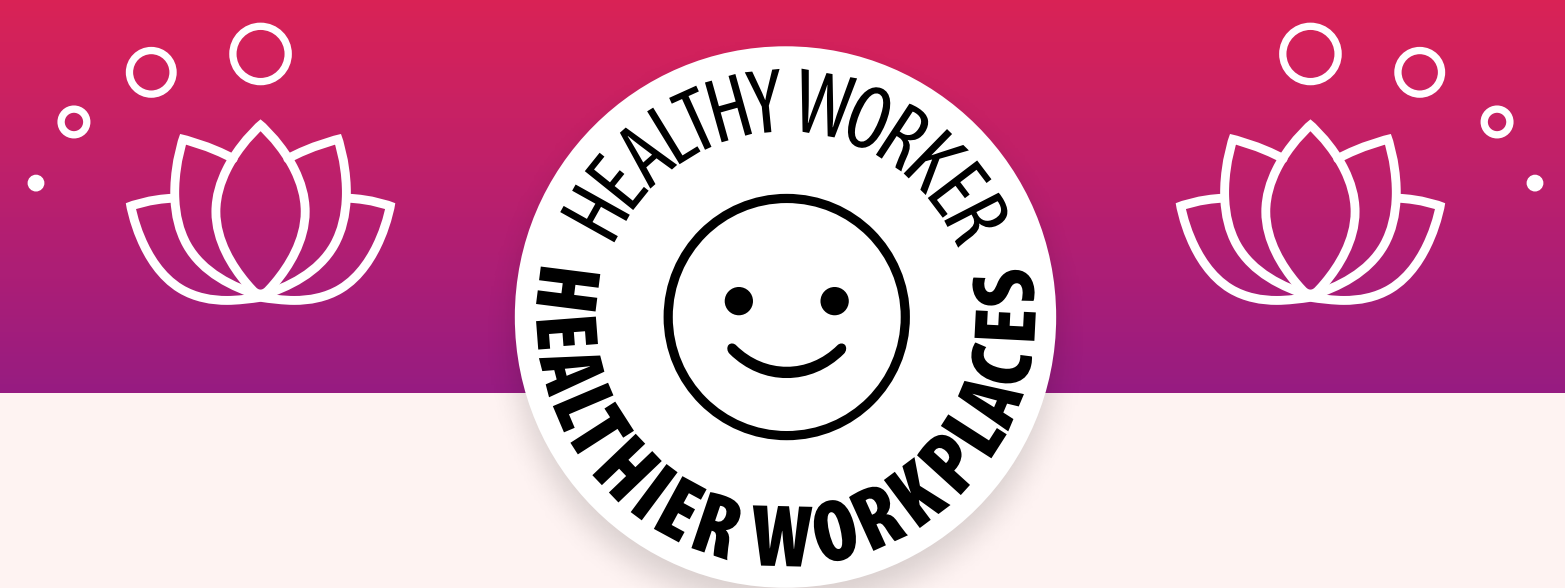

\section{Keep yourself healthy during your Period!}

\section{?}

How can WOMEN WORKERS keep themselves healthy during menstruation?

\section{!}

Learn what to do for YOUR PERIOD and how to feel better:

— USE A CLEAN CLOTH OR SANITARY PAD

- CHANGE YOUR CLOTH OR PAD EVERY 2 TO 6 HOURS

- RELIEVE PAIN BY STRETCHING, USING A WARM COMPRESS, OR TAKING A PAIN RELIEVER

- DISPOSE OF WASTE PROPERLY

— EAT YOUR NORMALLY HEALTHY DIET

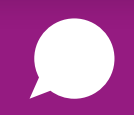

Talk to a health care provider you trust at your workplace or in your community 
○
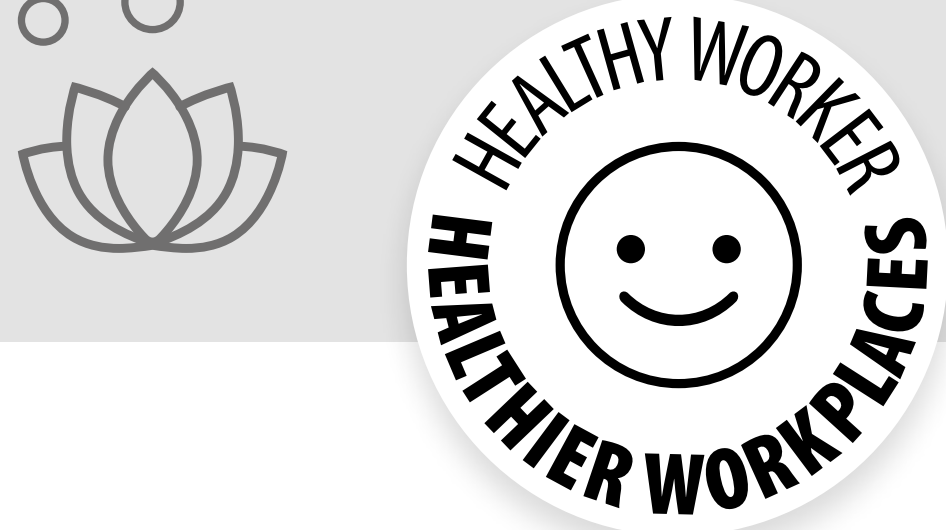

\section{Keep yourself healthy during your Period!}

\section{?}

How can WOMEN WORKERS keep themselves

healthy during menstruation?

\section{!}

Learn what to do for YOUR PERIOD and how to feel better:

— USE A CLEAN CLOTH OR SANITARY PAD

- CHANGE YOUR CLOTH OR PAD EVERY 2 TO 6 HOURS

- RELIEVE PAIN BY STRETCHING, USING A WARM COMPRESS, OR TAKING A PAIN RELIEVER

- DISPOSE OF WASTE PROPERLY

— EAT YOUR NORMALLY HEALTHY DIET

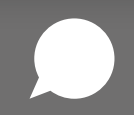

Talk to a health care provider you trust at your workplace or in your community 

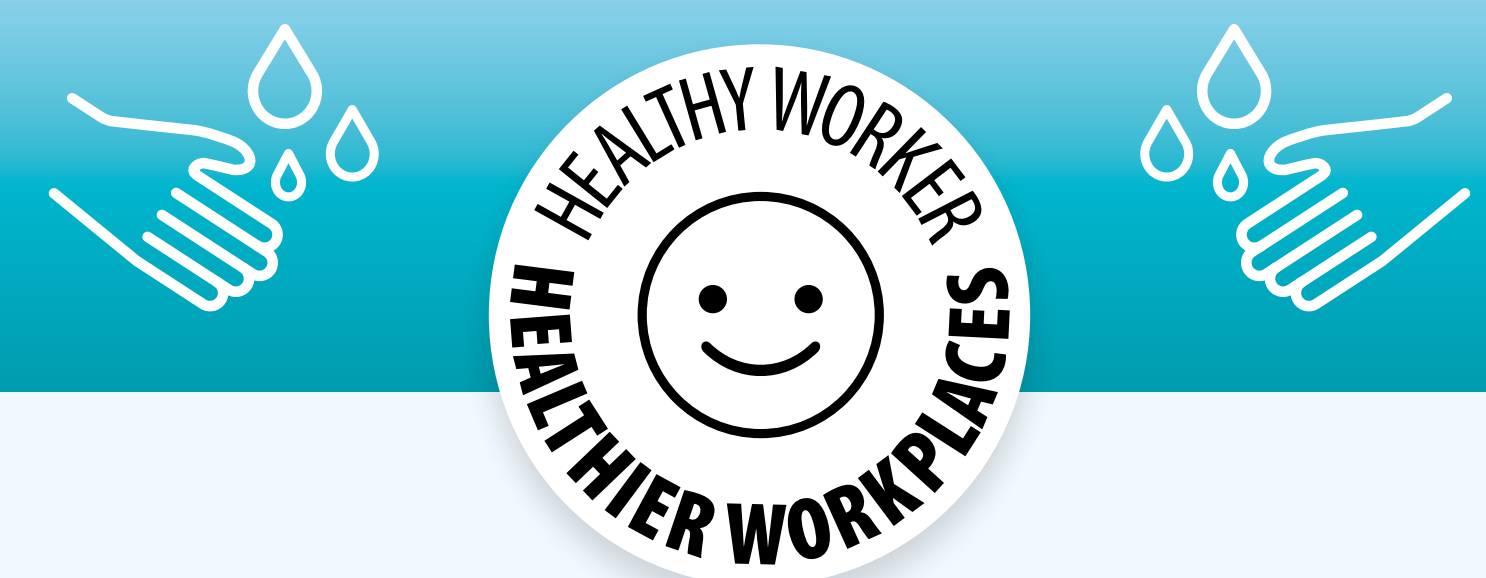

\section{Wash your Hands!}

\section{WHEN?}

(V) After using the tOILet

(1) BEFORE AND AFTER PREPARING FOOD

(V) Before eating FOOD

(V) after handling raw meat

(V) AFter BLOWING YOUR NOSE, COUGHING, OR SNEEZING

(V) Before ANd AFter CARING FOR SOMEONE WHO IS SICK
(V) before and after treating A CUT OR WOUND

(V) after changing a baby Or Cleaning UP A CHILD WHO HAS USED THE TOILET

(V) after touching An Animal, ANIMAL FEED, OR ANIMAL WASTE

You should wash your hands to prevent the spread of germs and stay healthy.

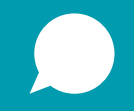

Talk to a health care provider you trust at your workplace or in your community 

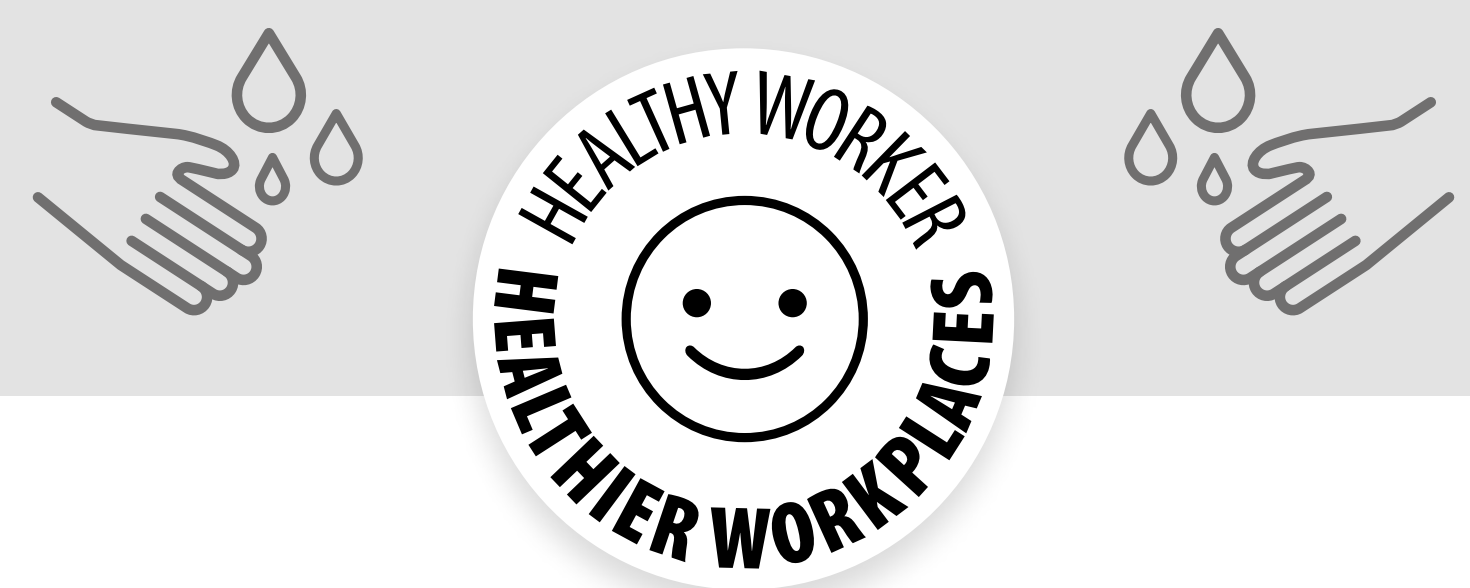

\section{Wash your Hands!}

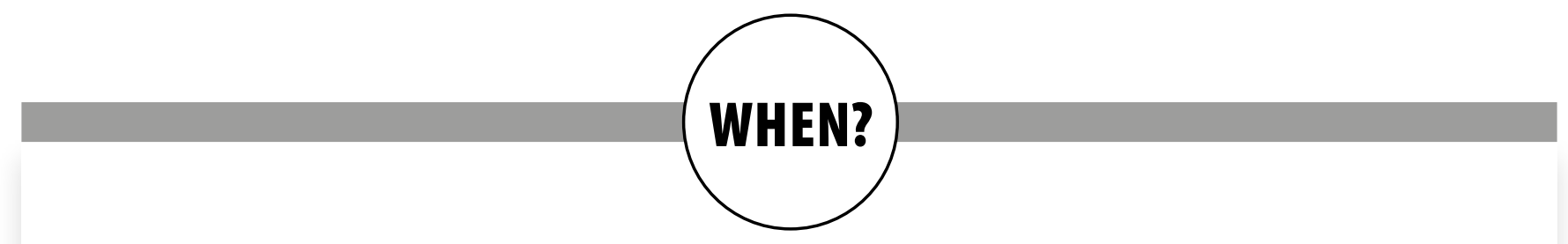

(V) after using the tollet

(1) Before and after preparing FOOd

(V) before eating food

(V) after handling raw meat

(V) after blowing YOUR NOSE, COUGHING, OR SNEEZING

(1) Before and after Caring FOR SOMEONE WHO IS SICK
(V) before and after treating A CUT OR WOUND

(1) after changing a baby or Cleaning UP A CHILD WHO HAS USED THE TOILET

(V) after touching an Animal, ANIMAL FEED, OR ANIMAL WASTE

You should wash your hands to prevent the spread of germs and stay healthy. 


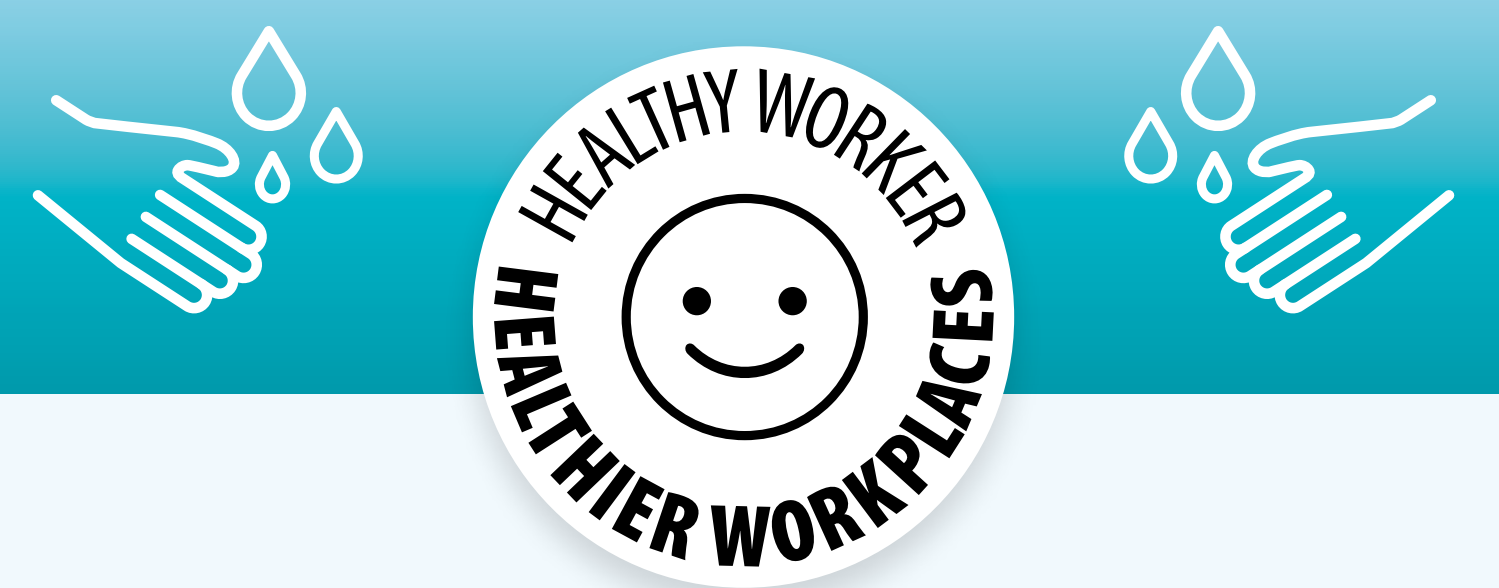

\section{Wash your Hands!}
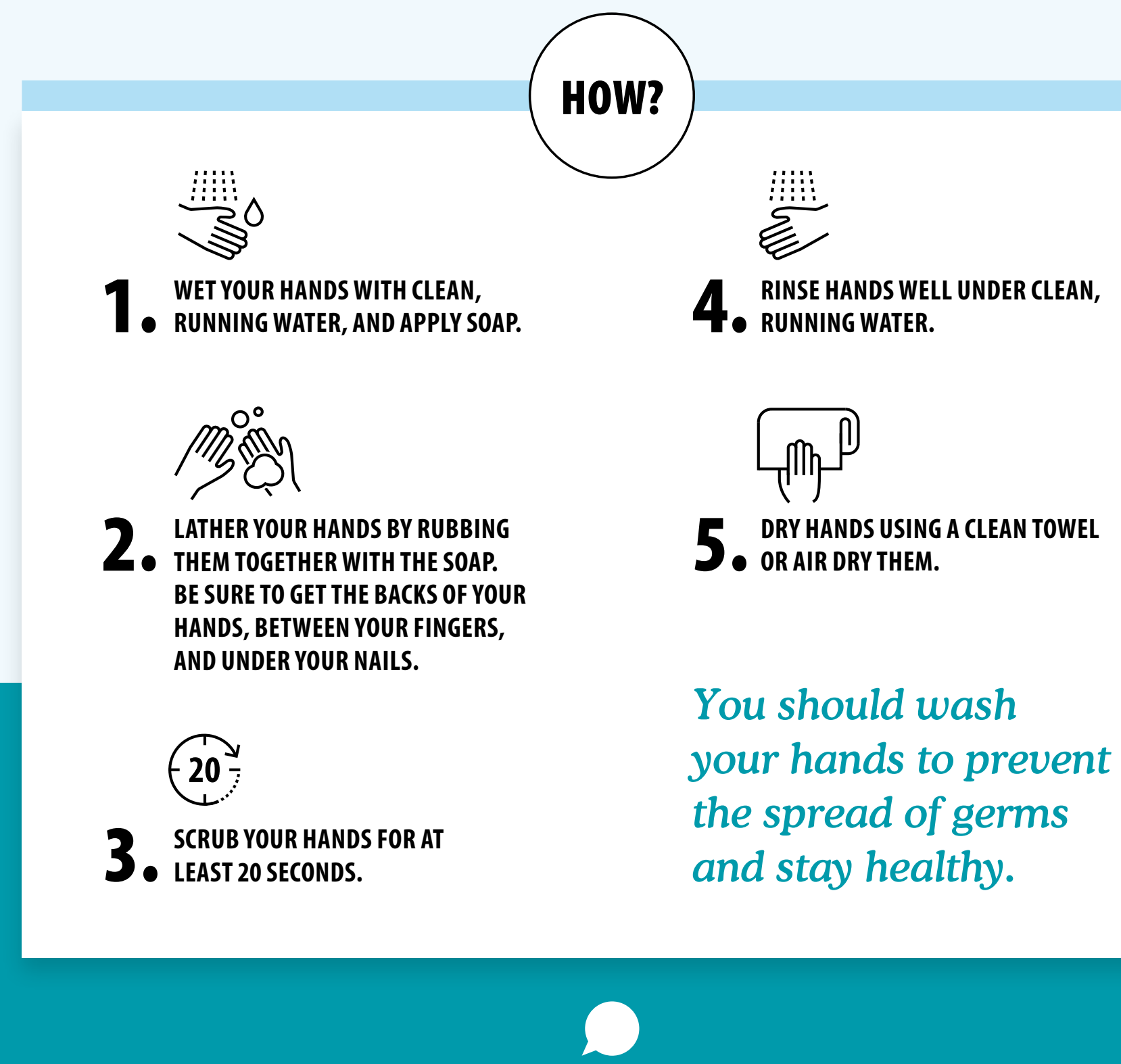

Talk to a health care provider you trust at your workplace or in your community 

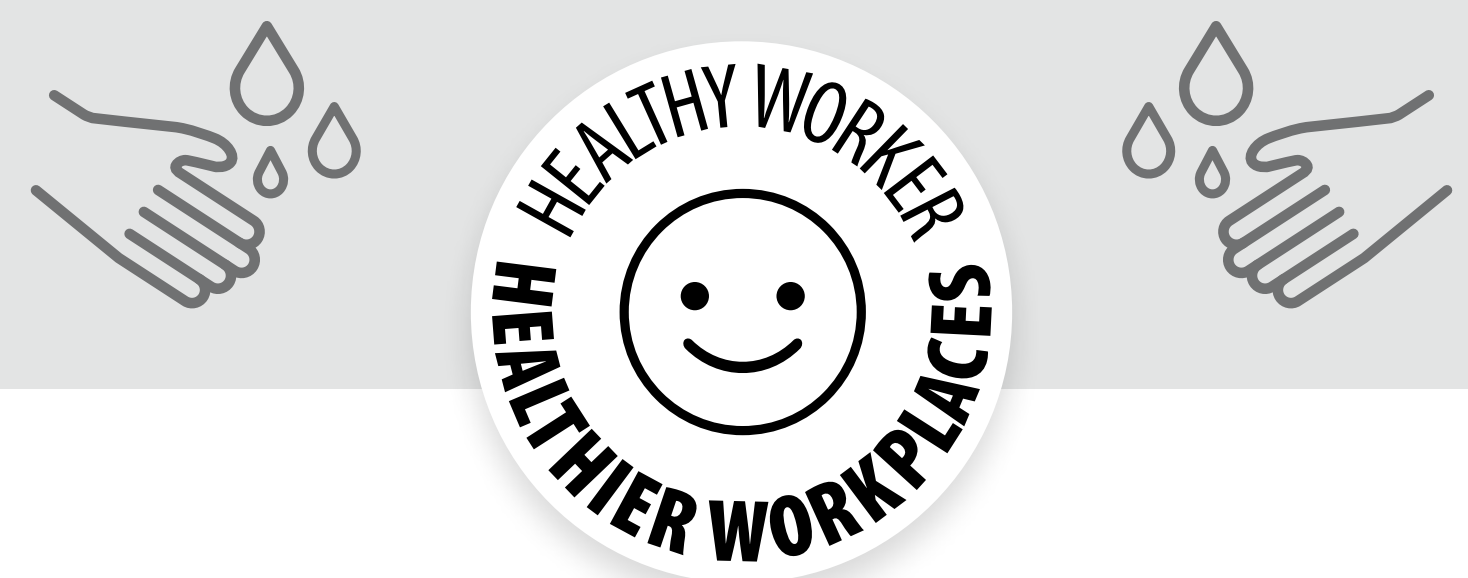

\section{Wash your Hands!}
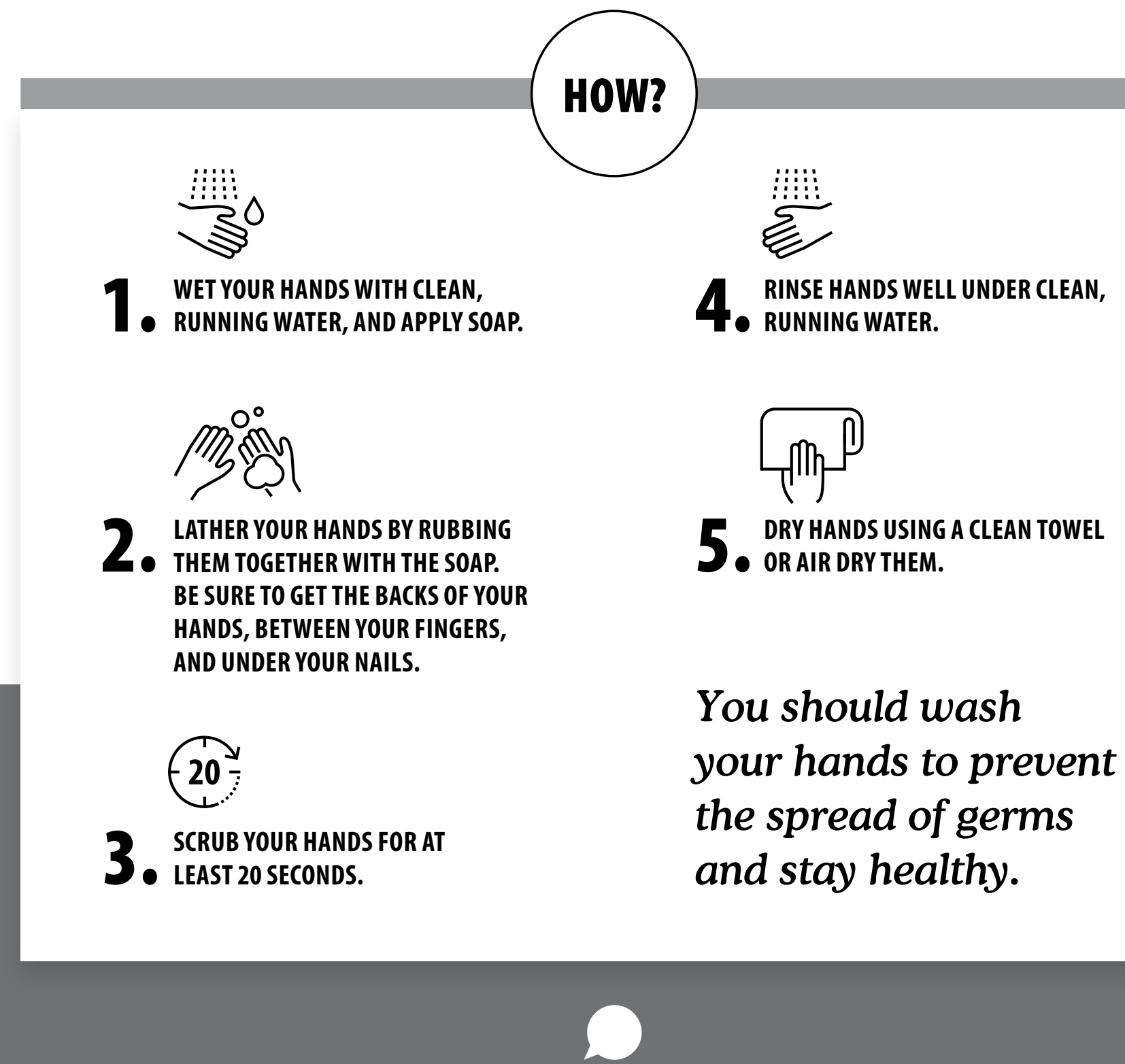

Talk to a health care provider you trust at your workplace or in your community 

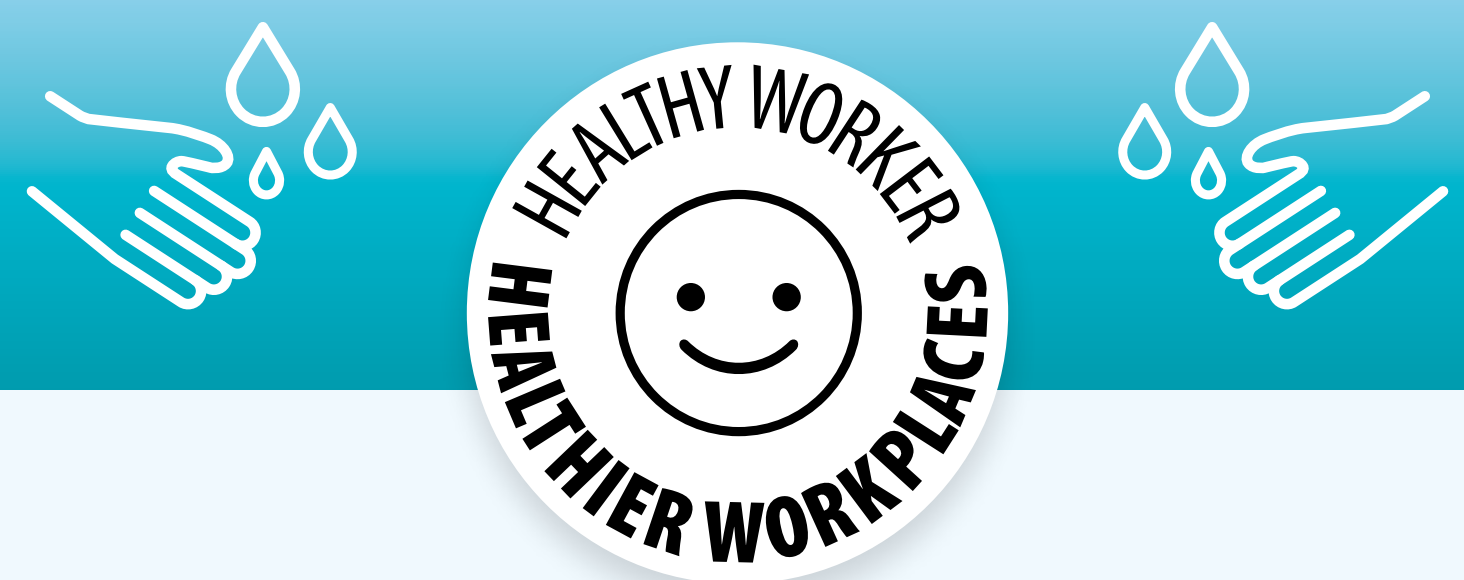

\section{Stop germs. Stay healthy. Wash your Hands!}

\section{?}

WHEN should you wash your hands?

\section{!}

There are IMPORTANT TIMES

to wash your hands:

- AFTER USING THE TOILET

- BEFORE AND AFTER PREPARING FOOD

- BEFORE EATING FOOD

- AFTER HANDLING RAW MEAT

- AFTER BLOWING YOUR NOSE, COUGHING, OR SNEEZING

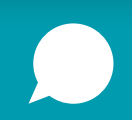

Talk to a health care provider you trust at your workplace or in your community 

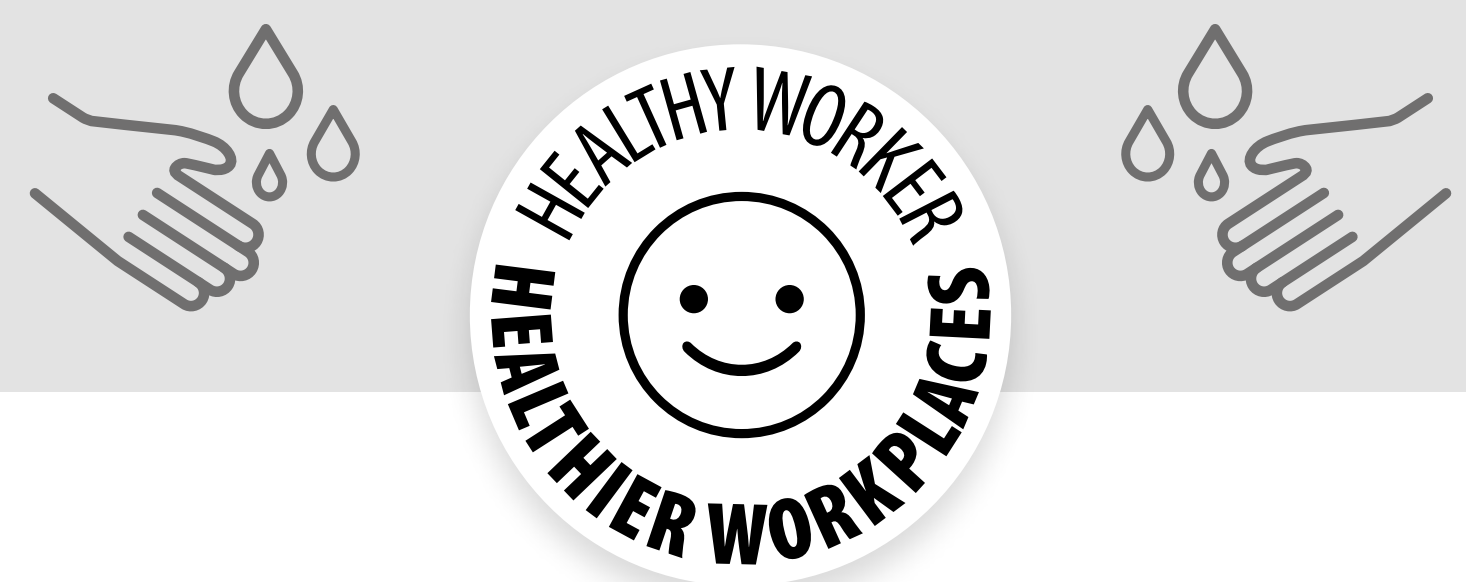

\section{Stop germs. Stay healthy. Wash your Hands!}

\section{?}

WHEN should you wash your hands?

\section{!}

There are IMPORTANT TIMES

to wash your hands:

- AFTER USING THE TOILET

— BEFORE AND AFTER PREPARING FOOD

- BEFORE EATING F00D

- AFTER HANDLING RAW MEAT

- AFTER BLOWING YOUR NOSE, COUGHING, OR SNEEZING

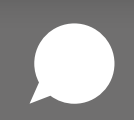

Talk to a health care provider you trust at your workplace or in your community 

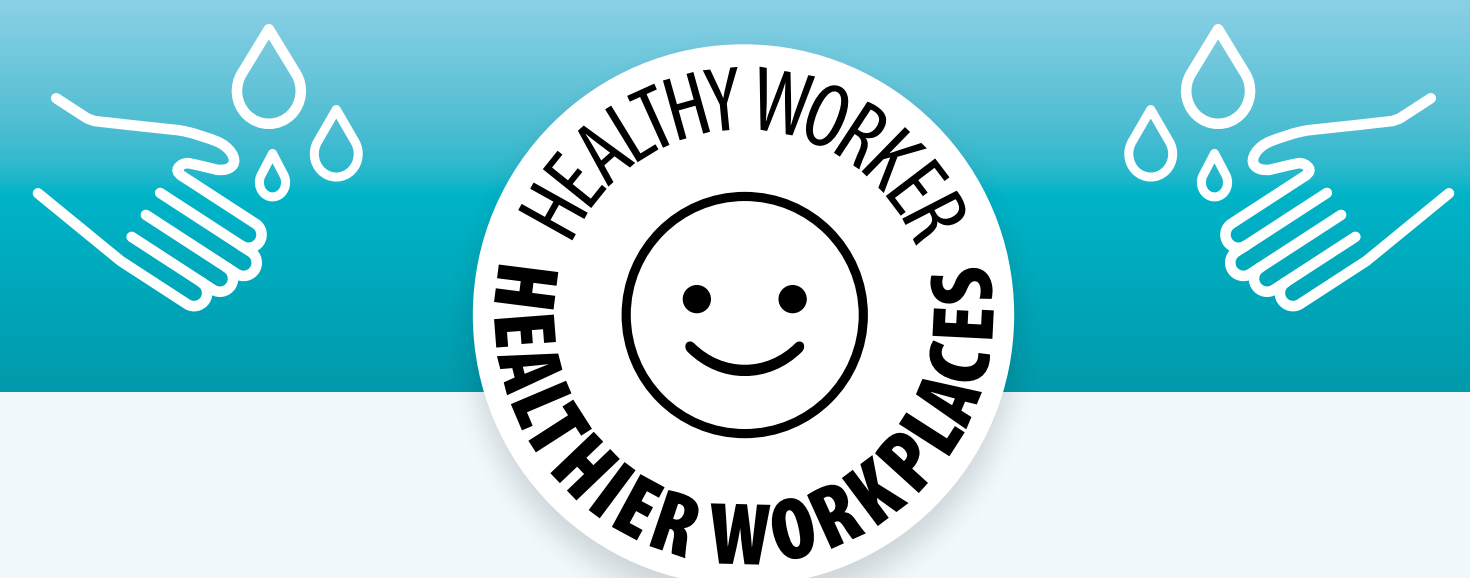

Do you know the

Facts about handwashing? Check your knowledge:
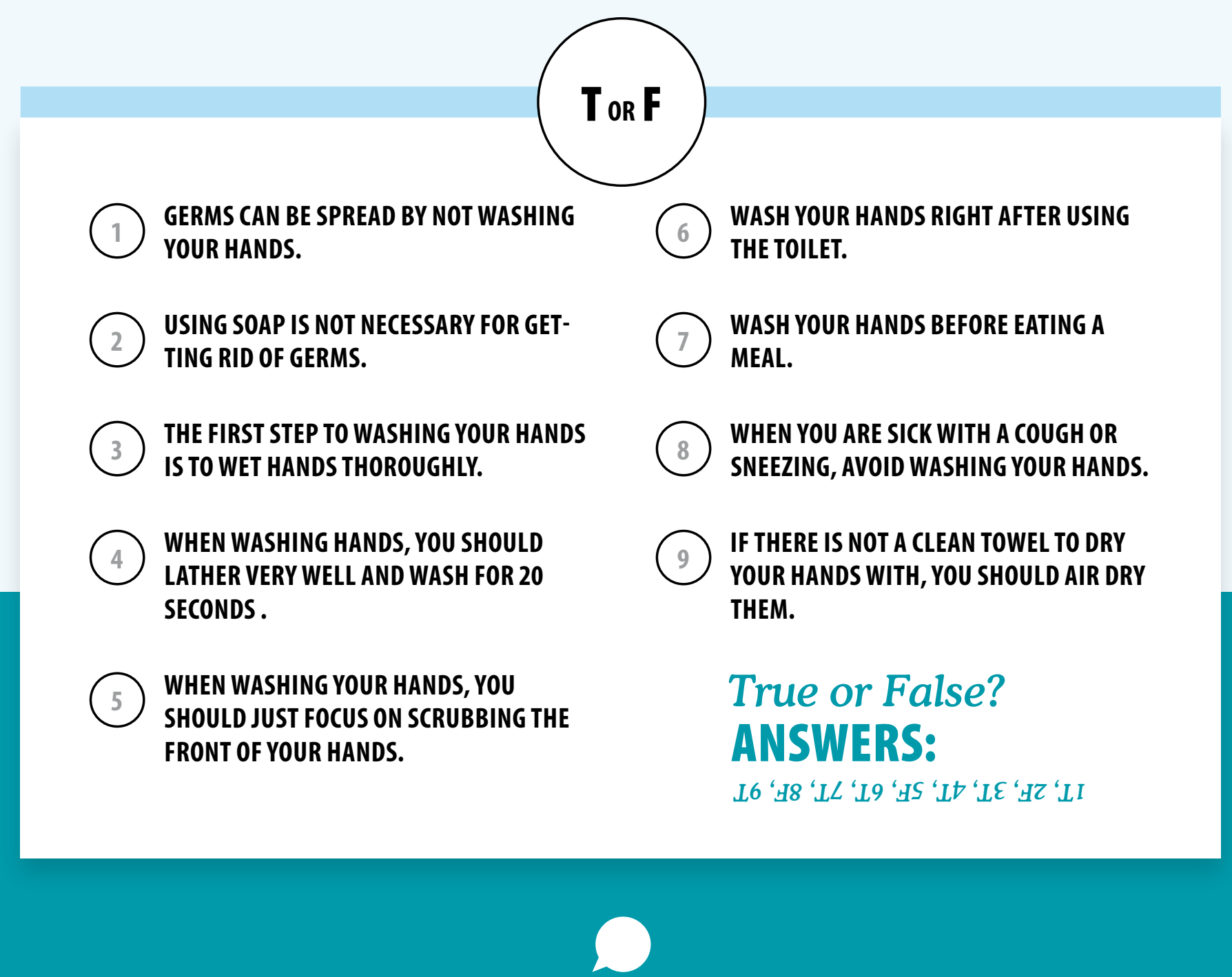

Talk to a health care provider you trust at your workplace or in your community 

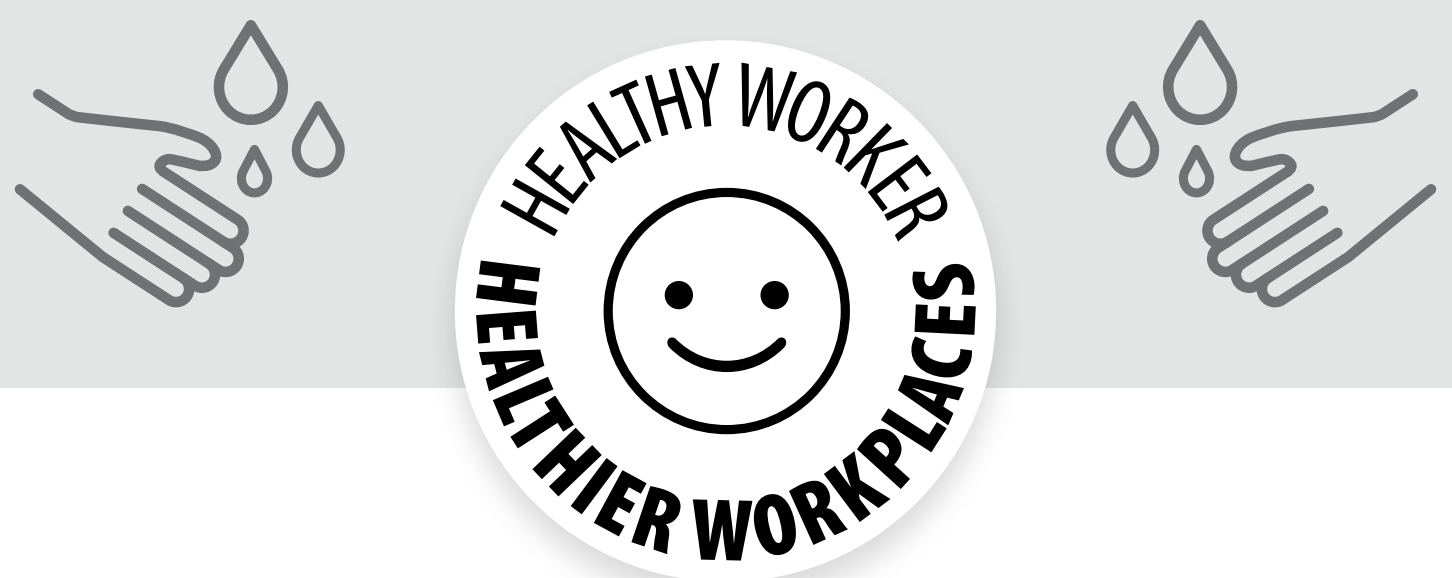

\section{Do you know the Facts about handwashing? Check your knowledge:}

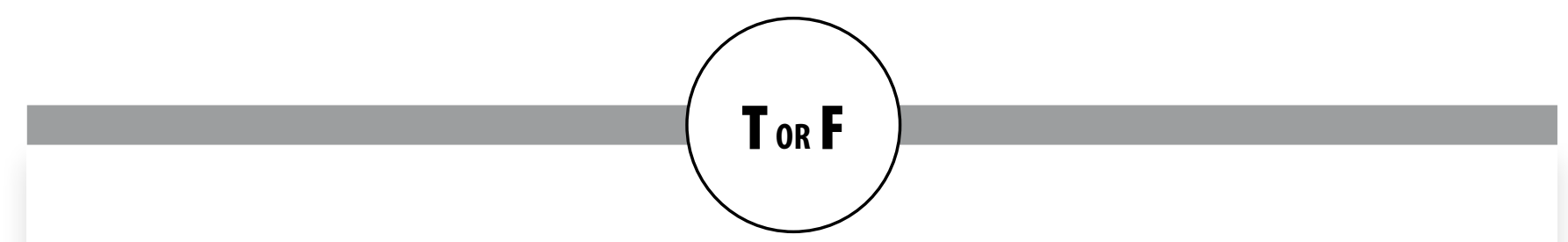

(1) GERMS CAN BE SPREAD BY NOT WASHING YOUR HANDS. WASH YOUR HANDS RIGHT AFTER USING
THE TOILET. (2) USING SOAP IS NOT NECESSARY FOR GET-
TING RID OF GERMS.

(7) WASH YOUR HANDS BEFORE EATING A
MEAL.

(3) THE FIRST STEP TO WASHING YOUR HANDS IS TO WET HANDS THOROUGHLY.

(8) WHEN YOU ARE SICK WITH A COUGH OR
SNEEZING, AVOID WASHING YOUR HANDS.

(4) WHEN WASHING HANDS, YOU SHOULD LATHER VERY WELL AND WASH FOR 20 SECONDS.

9 IF THERE IS NOT A CLEAN TOWEL TO DRY YOUR HANDS WITH, YOU SHOULD AIR DRY THEM.

True or False? ANSWERS:

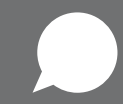

Talk to a health care provider you trust at your workplace or in your community 

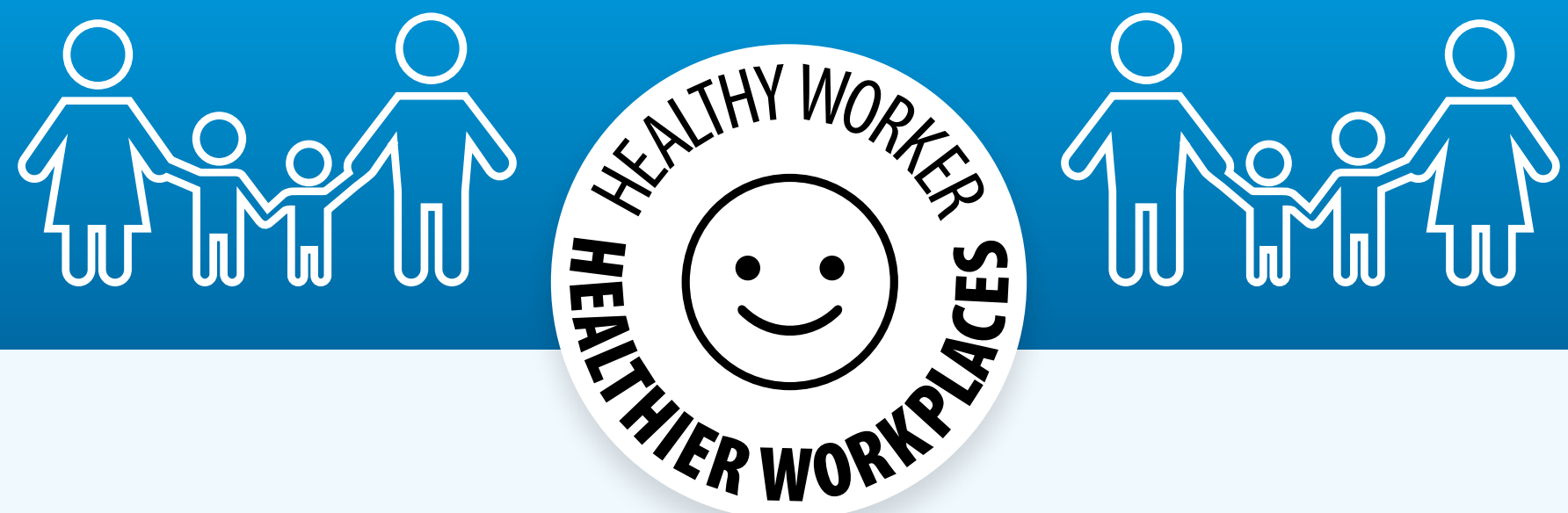

\section{When you take care of your family's health, you take care of your own health!}

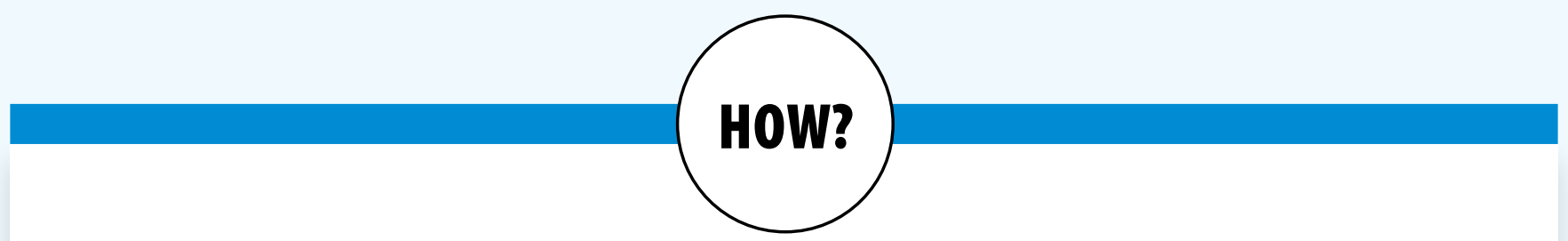

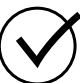

\section{PLAN AND SPACE YOUR FAMILY} WITH YOUR PARTNER

You and your partner should decide the right number of children for your family. Wait at least 2 years after your last child's birth before trying to become pregnant again. This spacing will help significantly increase the chances of a safe birth for mother and child. A healthy birth will give your children a better start at school and in life. A planned family has more money to spend.

\section{TAKE YOUR CHILDREN TO} THE HEALTH CLINIC

Getting your children vaccinated and keeping them healthy is one of the most important things that you can do as a father. You are critically important in protecting your children from illnesses.

\section{PARTICIPATE IN CARE-TAKING FOR} YOUR FAMILY

You can show you care by staying interested in your children's schooling and their health. It's important to play and spend time with them, in addition to the role of providing financial support to your family.

\section{A healthier family means a healthier you. Being an involved parent is good for you.}

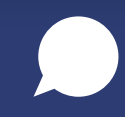

Talk to a health care provider you trust at your workplace or in your community 

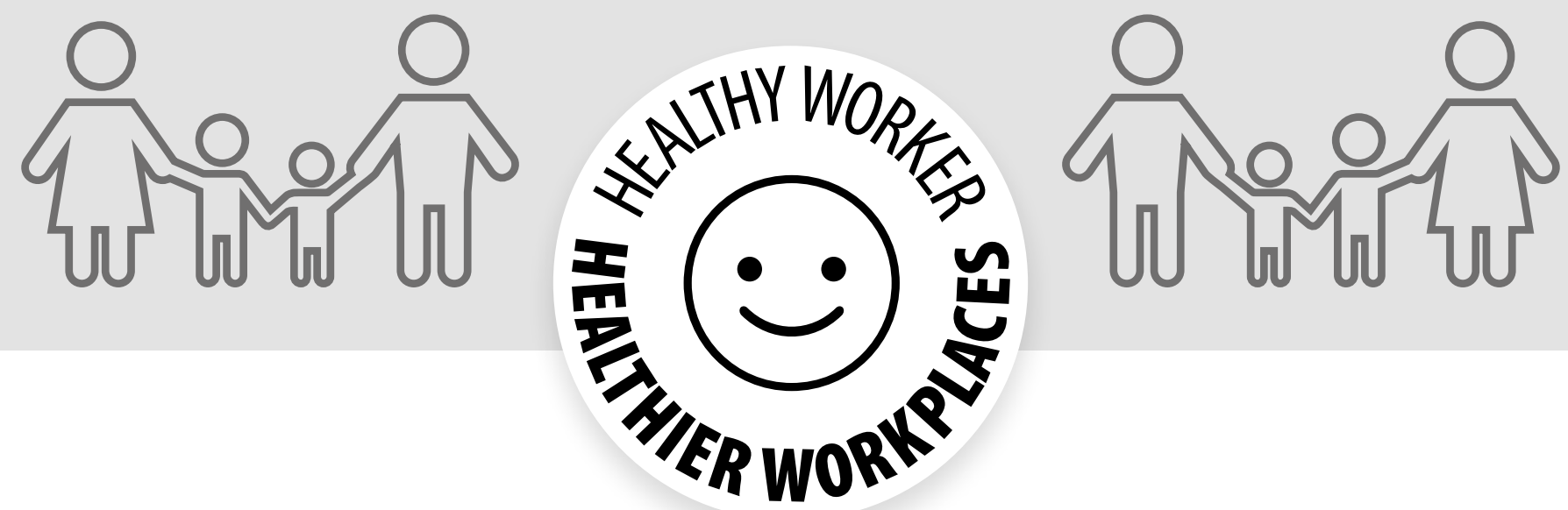

\section{When you take care of your family's health, you take care of your own health!}

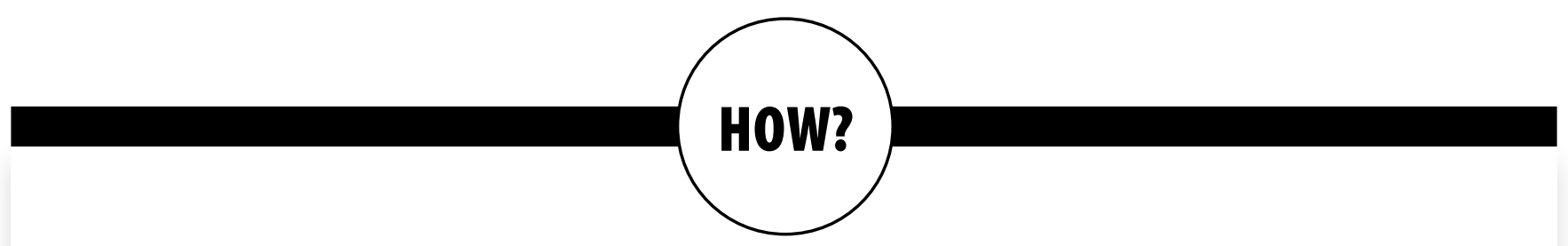

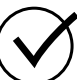

PLAN AND SPACE YOUR FAMILY WITH YOUR PARTNER

You and your partner should decide the right number of children for your family. Wait at least 2 years after your last child's birth before trying to become pregnant again. This spacing will help significantly increase the chances of a safe birth for mother and child. A healthy birth will give your children $a$ better start at school and in life. A planned family has more money to spend.

TAKE YOUR CHILDREN TO THE HEALTH CLINIC

Getting your children vaccinated and keeping them healthy is one of the most important things that you can do as a father. You are critically important in protecting your children from illnesses.

\section{PARTICIPATE IN CARE-TAKING FOR} YOUR FAMILY

You can show you care by staying interested in your children's schooling and their health. It's important to play and spend time with them, in addition to the role of providing financial support to your family.

\section{A healthier family} means a healthier you. Being an involved parent is good for you. 


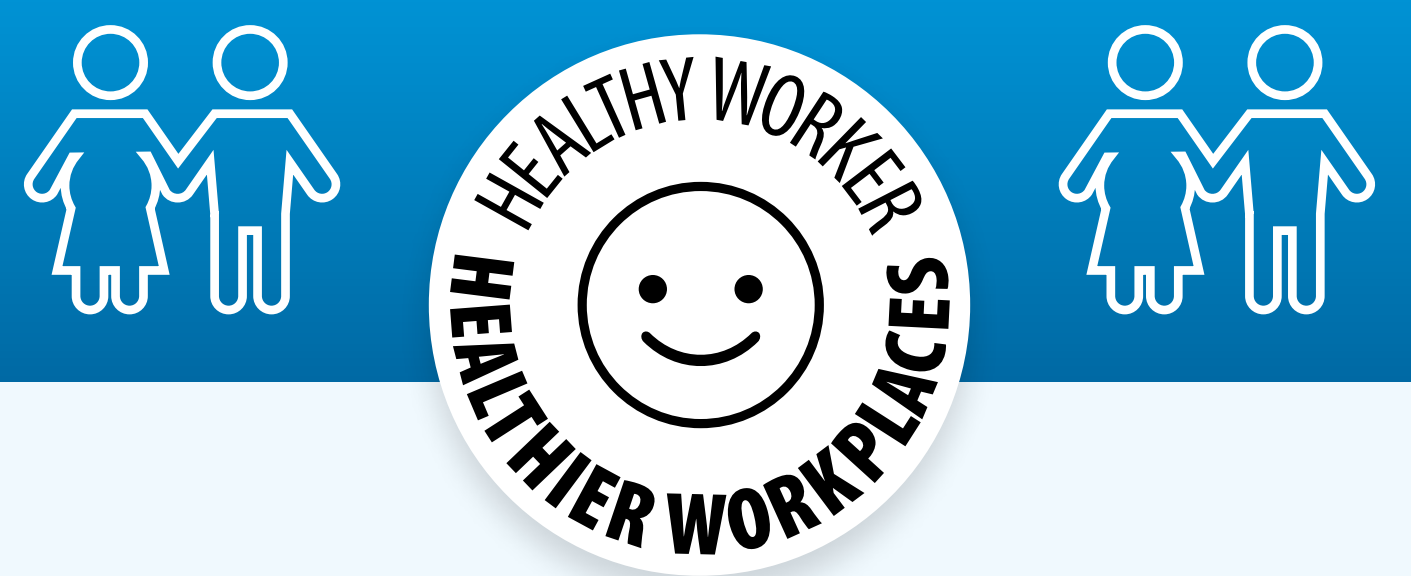

\section{Fathers can care for the health of their families!}

\section{?}

How can MEN WORKERS give their families a good start?

\section{!}

BE INVOLVED from the start

— PLAN THE SIZE OF YOUR FAMILY WITH YOUR PARTNER

- SPACE PREGNANCIES FOR HEALTHIER MOM, BABY, AND FAMILY

— SUPPORT THE MOTHER DURING PREGNANCY

- ATTEND PRE-NATAL VISITS

\section{D}

Talk to a health care provider you trust at your workplace or in your community 

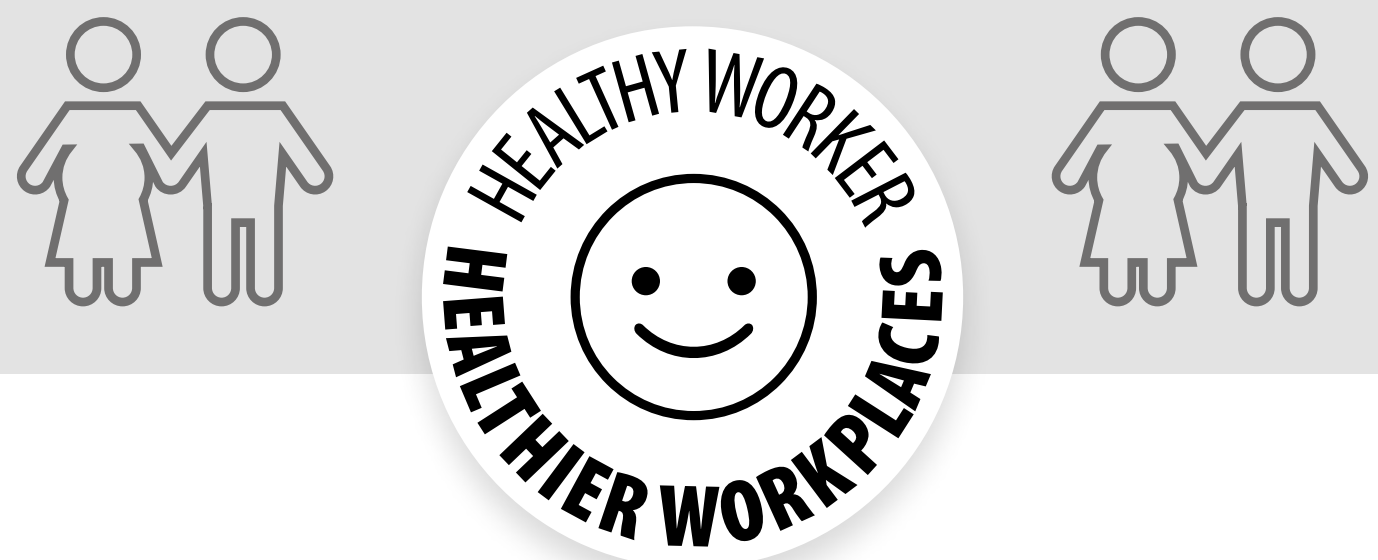

\section{Fathers can care for the health of their families!}

\section{$?$}

How can MEN WORKERS give their families a good start?

\section{!}

BE INVOLVED from the start

— PLAN THE SIZE OF YOUR FAMILY WITH YOUR PARTNER

- SPACE PREGNANCIES FOR HEALTHIER MOM, BABY, AND FAMILY

— SUPPORT THE MOTHER DURING PREGNANCY

- ATTEND PRE-NATAL VISITS

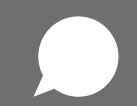

Talk to a health care provider you trust at your workplace or in your community 

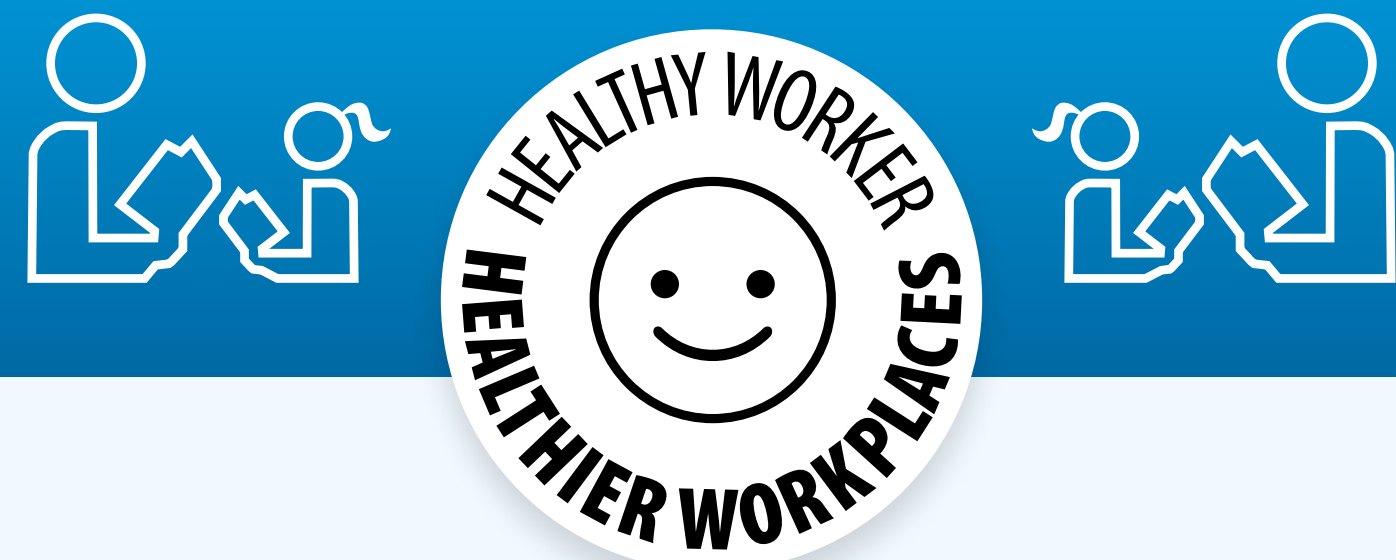

Fathers can care for the health of their families!

\section{?}

How can MEN WORKERS take care of their families?

\section{!}

SHARE the care work

- SEND YOUR CHILDREN TO SCHOOL

- PLAY AND SPEND TIME WITH YOUR CHILDREN

— TAKE YOUR CHILDREN TO THE HEALTH CLINIC

- GET YOUR CHILDREN VACCINATED

- RESOLVE FAMILY CONFLICT PEACEFULLY

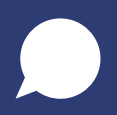

Talk to a health care provider you trust at your workplace or in your community 

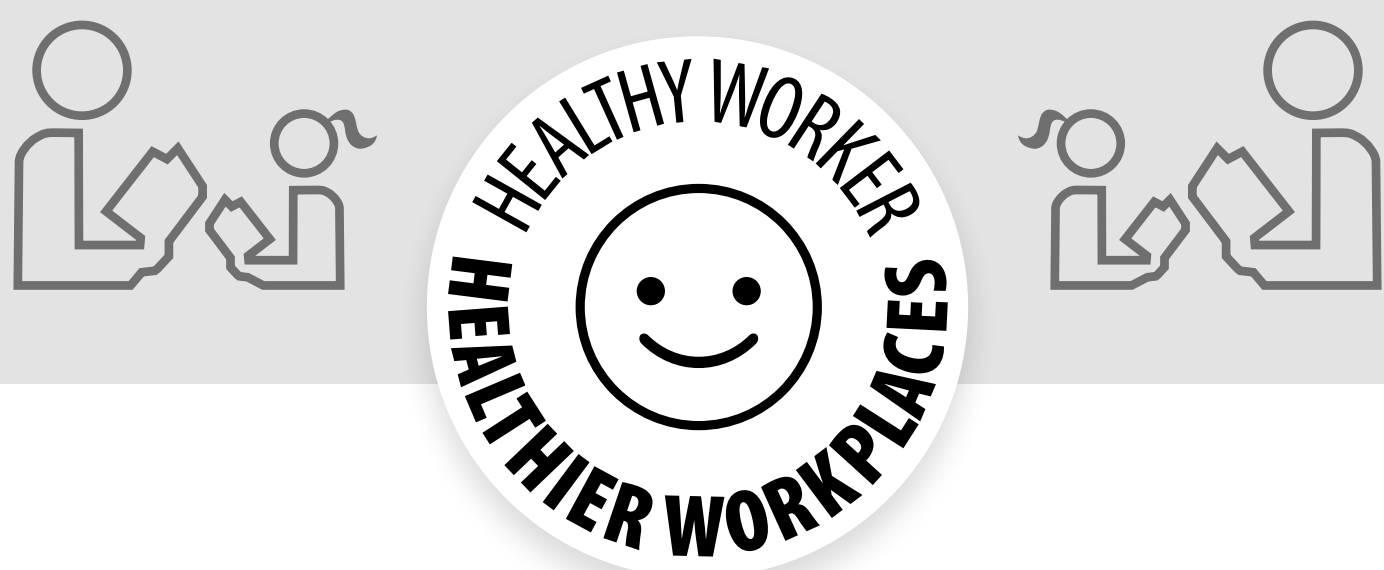

\section{Fathers can care for the health of their families!}

\section{?}

How can MEN WORKERS take care of their families?

\section{!}

SHARE the care work

- SEND YOUR CHILDREN TO SCHOOL

- PLAY AND SPEND TIME WITH YOUR CHILDREN

- TAKE YOUR CHILDREN TO THE HEALTH CLINIC

- GET YOUR CHILDREN VACCINATED

- RESOLVE FAMILY CONFLICT PEACEFULLY

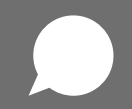

Talk to a health care provider you trust at your workplace or in your community 

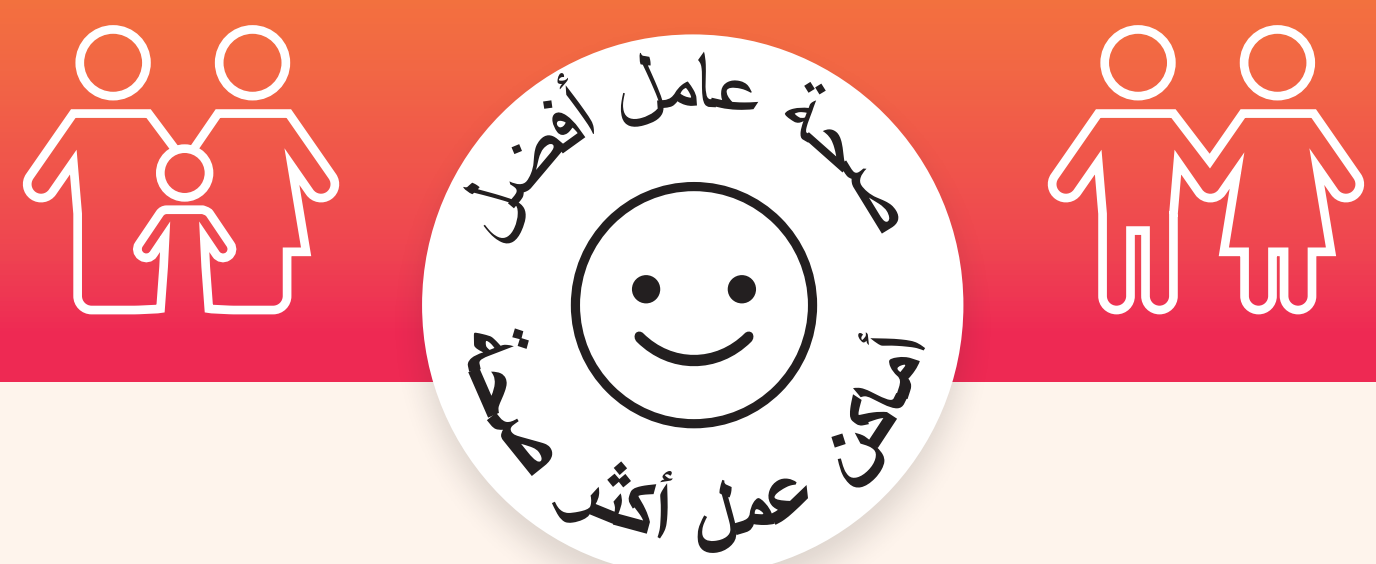

تنظيم الأسرة:

عن طريق الاختيار وليس الصدفة إل

(5)

ما أكثر طريقة فعالةلـ

$$
\text { - تنظيم أسرتك }
$$

- - منع حالات الحمل غير المرغوب بهات

- ـ تجنب حالات الإجهاض غير الآمنة

(!)

العاملات الإناث يستخدمن تتظيم الأسرة الحديث و الهرموني!
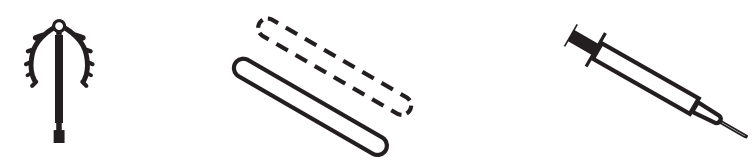

$\frac{000000000}{0000000000}$

اللولب

الزرع

الحقن

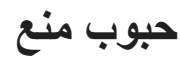

الحمل

\section{a \\ تحدث إلى مثدم الرعاية الصحية الذي ثثق فيه

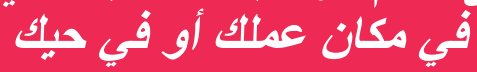




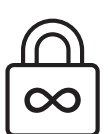

التعقيم

إلى الأبد

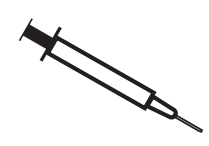

الحقن

- ب أنثن

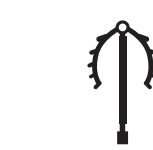

اللولب

oـ • المنوات

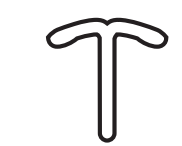

التولب الهرموني

r ـ - سنوات

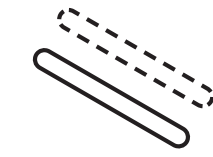

الز الزرع

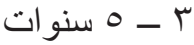

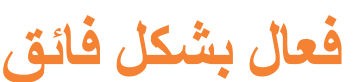

$* 99$

* عند الاستخدام النموذجي *

00

الو اقوي الذكري والأنثوي

يستخدم مرة و احدة

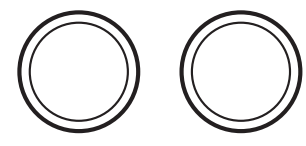

الحلقة المهبلية

كل شهر الرهي

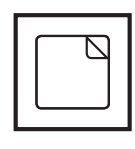

اللصقة

كل أسبوع
0000000000

حبوب منع الحمل

كل يوم

\section{قُعال للاغاية. \\ $* 9 \leq-91$ \\ *ند الاستخدام النمو ذجي}

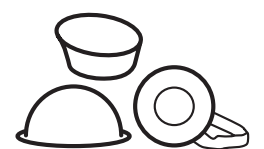

غطاء عنق الرحم، الحجاب

العازل الأنثتوي والإسفنج

كل مرة

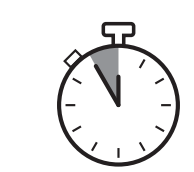

القذف الخارجي

كل مرة

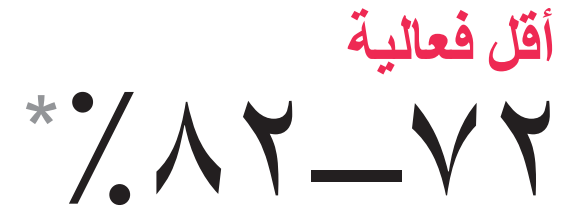

* عند الاستخدام النموذجي * 


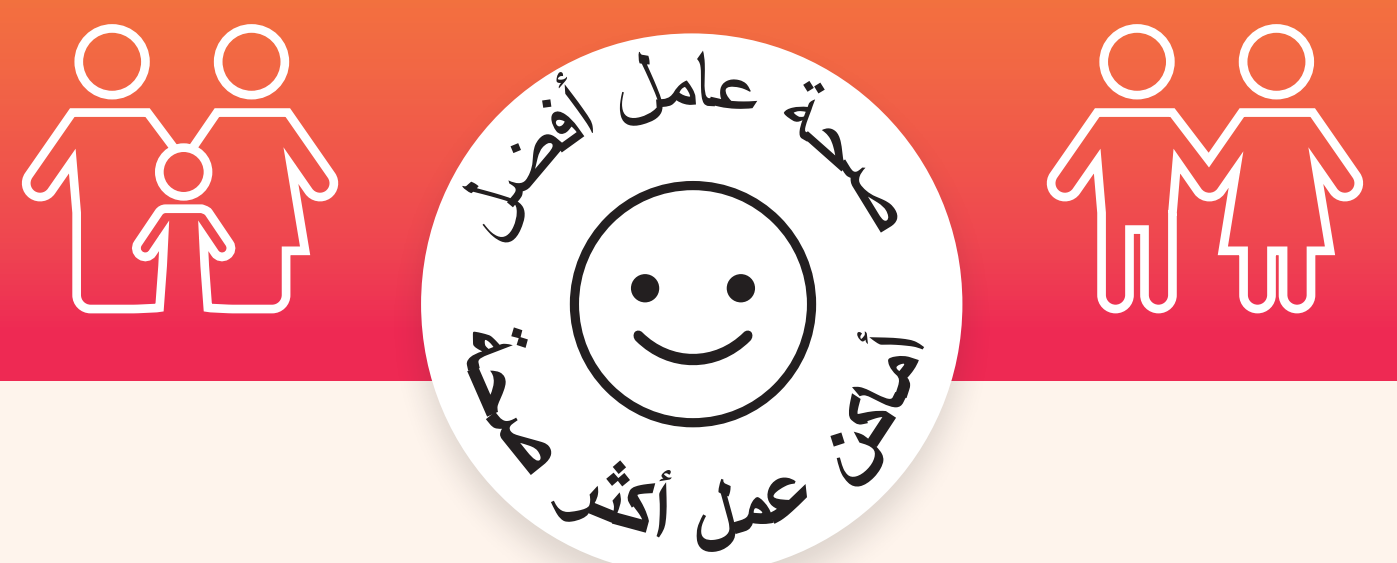

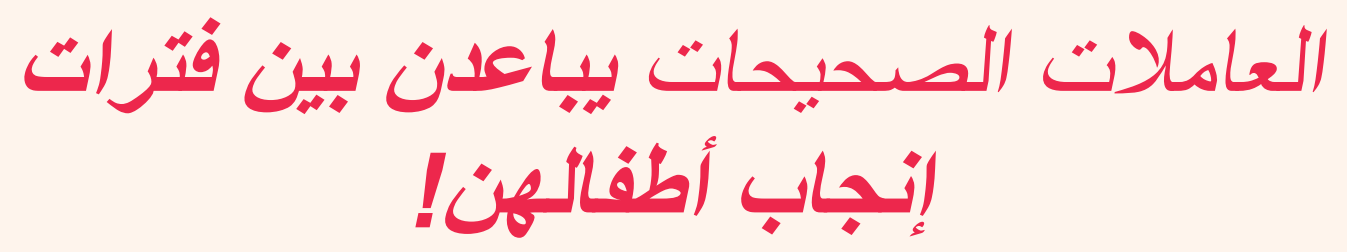

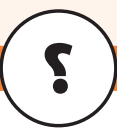

كيف تُنجبين طفلاً يتمتع بالصحة وتحظين بأسرة صحيحة؟

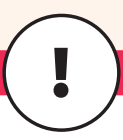

- - - انتظري حتى يبلغ عمر طقلت عامين قبل أن تحملي مرة أخرى.

- استخدمي طريقة حديثة لتنظيم الأسرة من اختيارك:
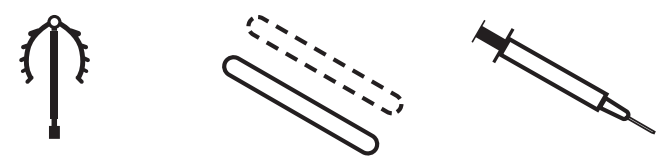

$\because 000000000$

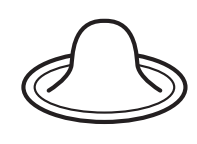

اللولب

الزرع

الحقن

حبوب منع

الحمل

الو اقوي

الأكري

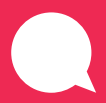

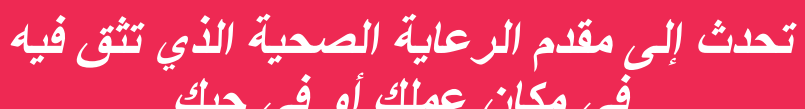

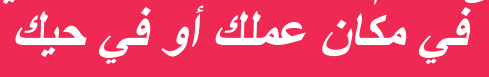



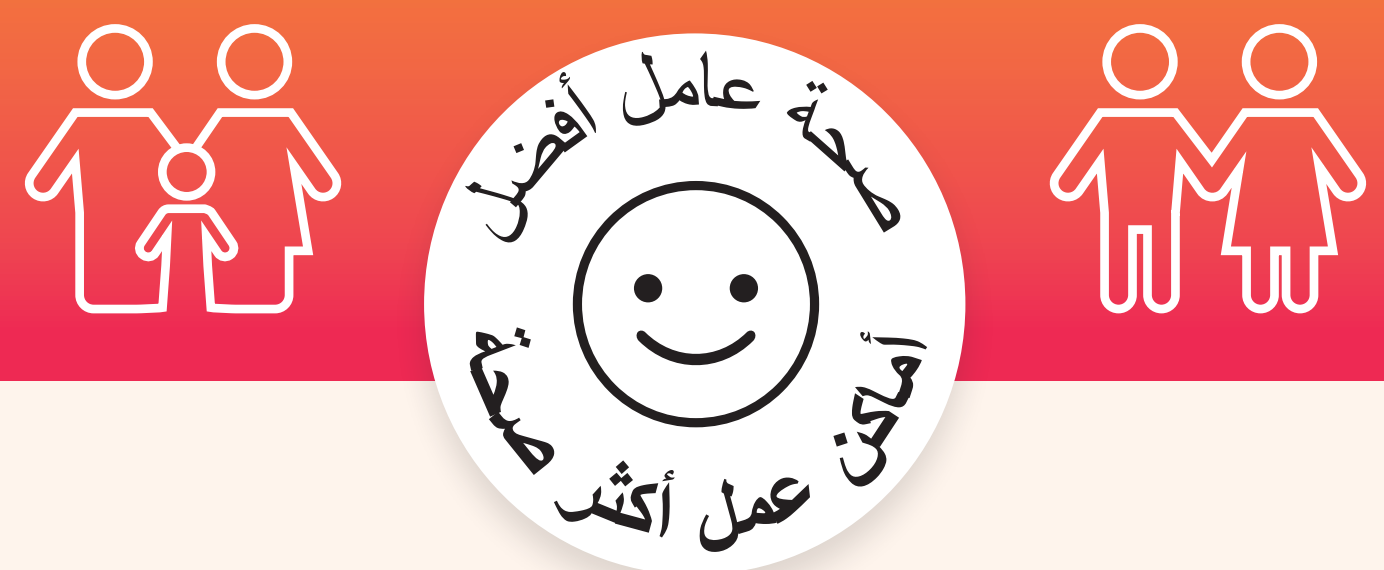

تمثنعي بعائلة أكثز صحة

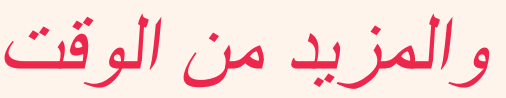

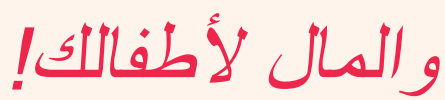

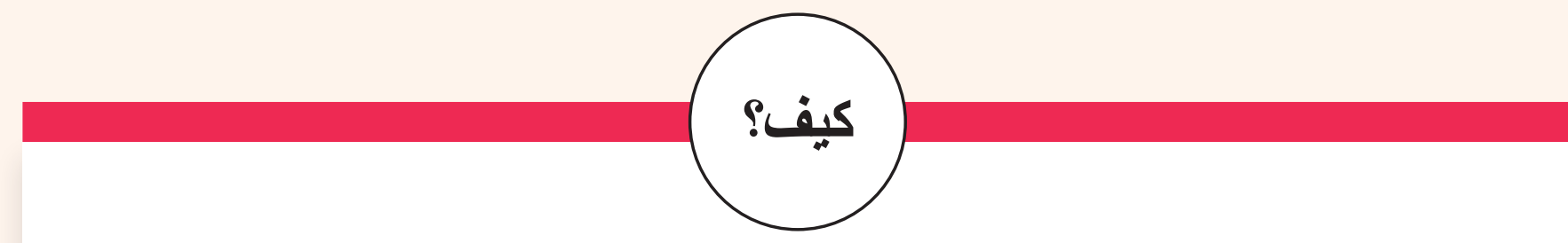

انتظري حتى بيلغ عدر طفلك عامبن قبل أن تحملي مرة أخرى.

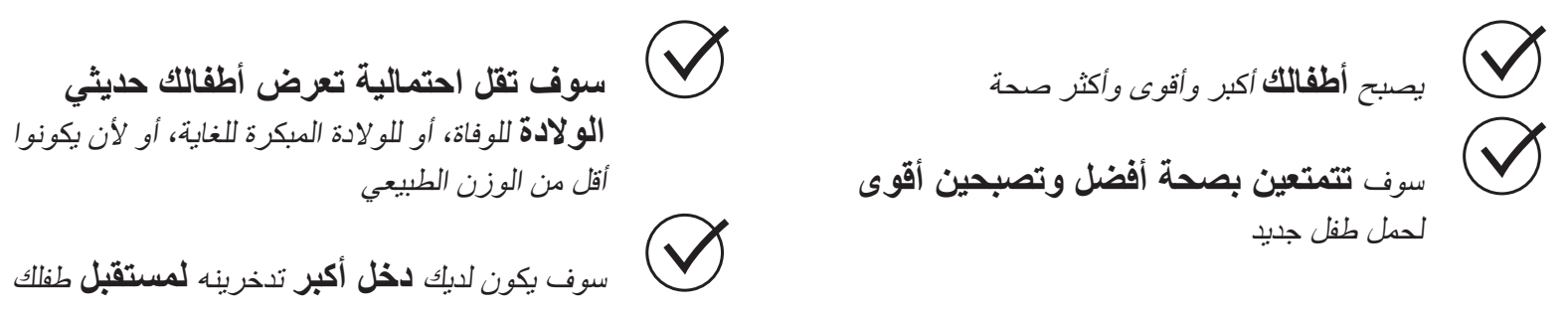

أي من طرق تنظيم الأسرة تناسبك؟
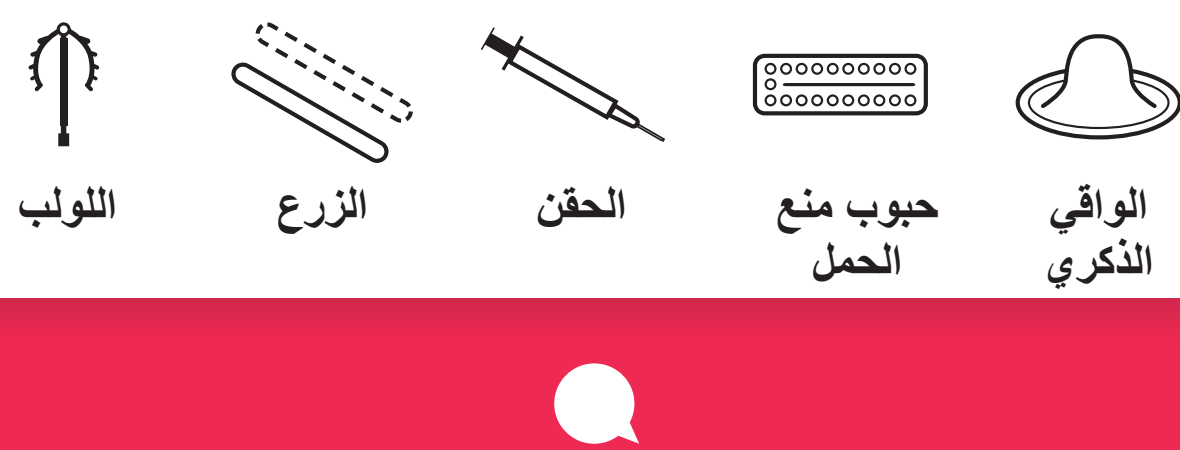

$$
\begin{aligned}
& \text { تحدث إلى مثدم الرعاية الصحية الذي ثثق فيه }
\end{aligned}
$$

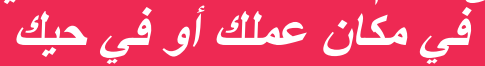


حمل لحالات الطوارئ. وهي أقوى من حبوب منع الحمل اليومية، ويجب أن تُستخدم نادرًا وفي لُوري

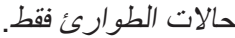

(!) الرضاعة الطبيعية تمنع الحمل حتى ستة

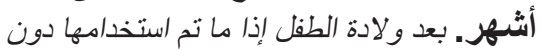

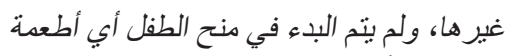

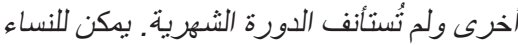
أيضا استخدام حبوب البروجستين فقط أثناء

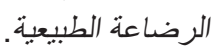

(! لن تسبب حبوب منع العمل عيويًا خلقية.

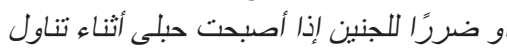

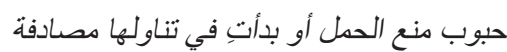

$$
\text { بعدما أصبحت حبلى بالفعل. }
$$

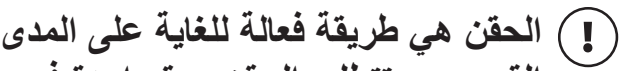

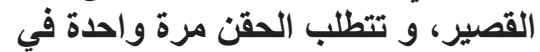

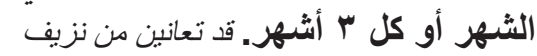

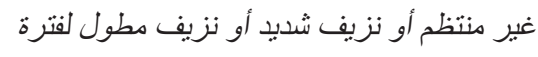

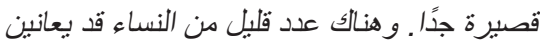

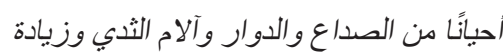

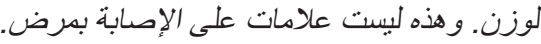
وغالبًا ما تزول هذه المشاكل خلال الأشهر القلبلة

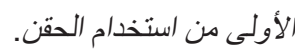

(? يجب أن تؤخذ حبوب منع الحمل باستمرار

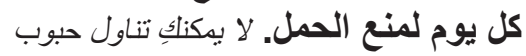

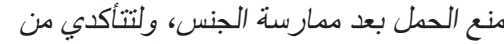

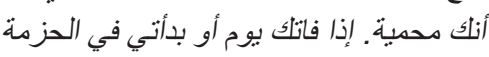

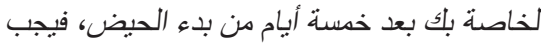

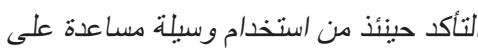
الأقل في الأيام السبعة اللاحقة. توجد حبوب مناعة منع
(!) موانع الحمل لا تسبب العقم. قد بيتغرق

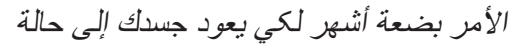

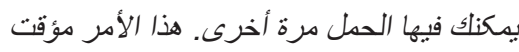

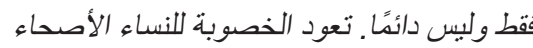
بغض النظر عن طول مدة استخدام الوسيلة

$$
\text { الهرمونية لتنظيم الأسرة. }
$$

(!) يعدُ اللولب وسيلة فعالة للغاية، يمكنها

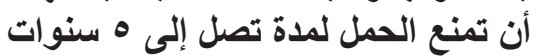

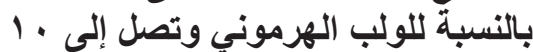
أعوام بالنسبة للولب النحابل النحسي. عندما يتم

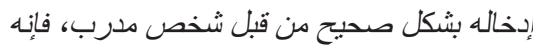

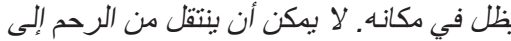

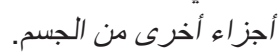

(!) وسائل منع الحمل الحيثة جيدة التحمل

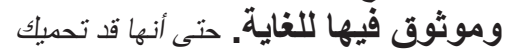

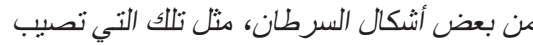

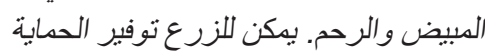
ضد مرض التهاب الحوض. لكن بُ بُنصح النساء

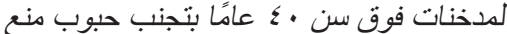
الحمل المركبة عن طريق الفم.
(إ) الفترات المتباعدة بين أطفالكك تعدُّ أفضل طريقة لضمان تمتع الطقل والأم والأسرة أفرة

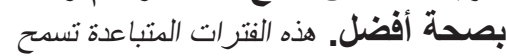

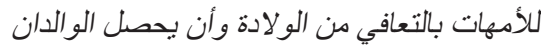

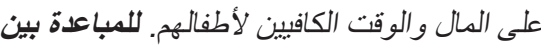
موعد أطفالك، استخذمي تنظيم الأسرة الحليث وانتظري عامين اثثين على الأقل بعد الولادة قبل الحبل محاولية الحمل مرة أخرى.

(!) من المككن أن تحملي أثناء الحيض. إذا

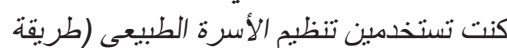

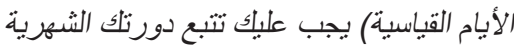
لعناية وألا تفترضي أنه لا بمكنك الحمل.

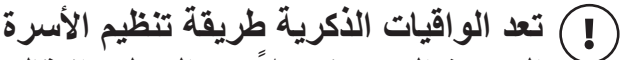

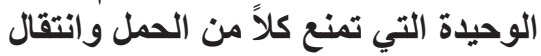

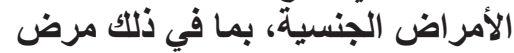
فيروس نقص المناعة البشرية (HIV) فئسية

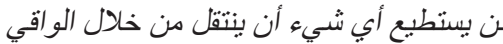

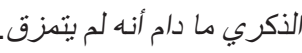



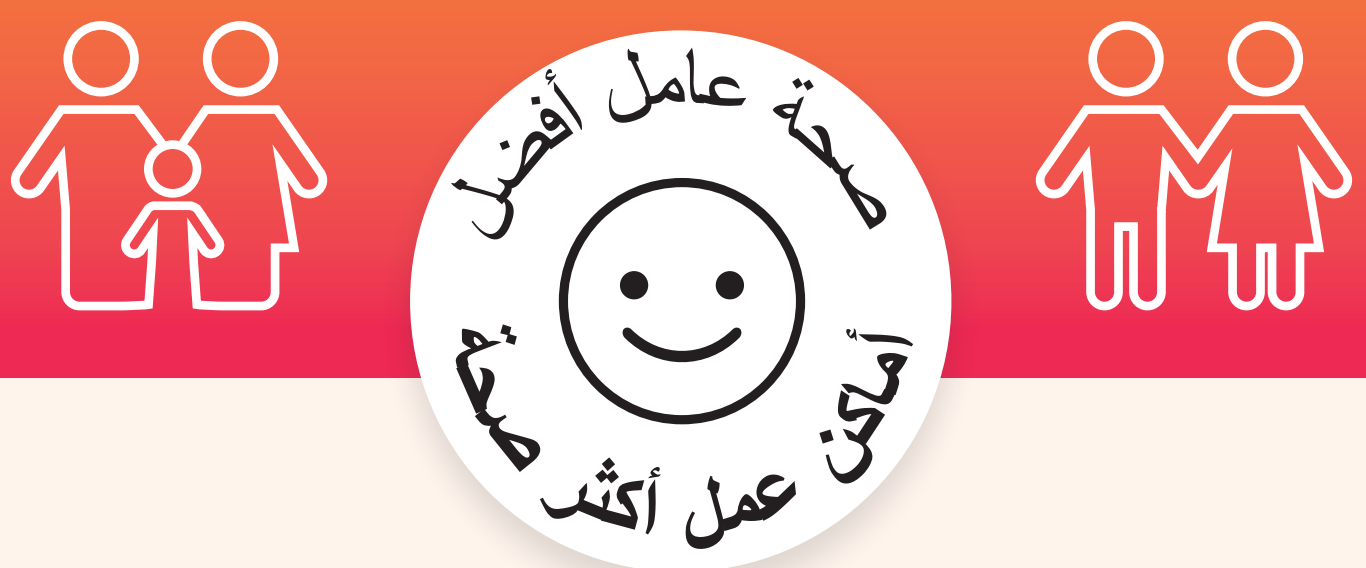

هل تعرفي الحقائق التي تنعلق

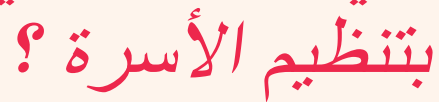

تعققي من معرفنتك :

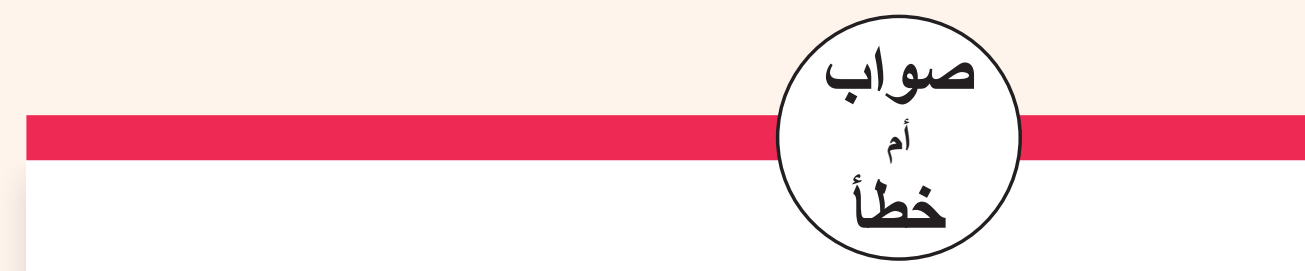

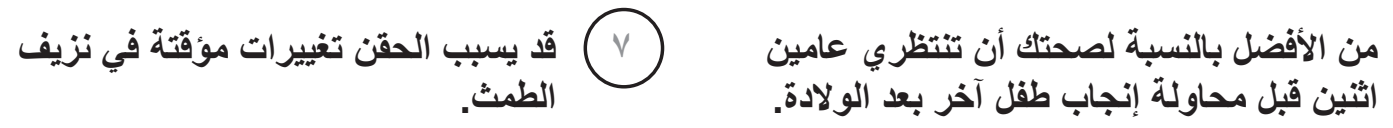

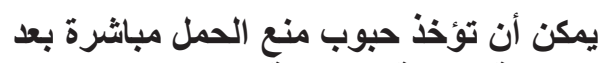
ممارسة العلاقة الحميمية (الجنس) وتمنع من

$$
\text { المرأة الحائض لا يمكن أن تحمل. }
$$

الحمل.

يعدُ الواقي الذكري هوٍ وسيلة منع الحمل

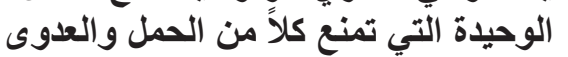

مجرد الرضاعة الطبيعية خلال الأشهر الستة الأولى تساعد على تجنب الحمل.

المنقولة جنسيًا (STIs).

بمكن أن تتسبب حبوب منع الحمل في الإصابة

يمكن للفائف اللولب أن تتحرك عبر الرحم وتثثب أعضاء الجسم.

صو اب أم خطأ? الإبإبـ

بمكن أن تتسبب حبوب منع الحمل في الإصابة 

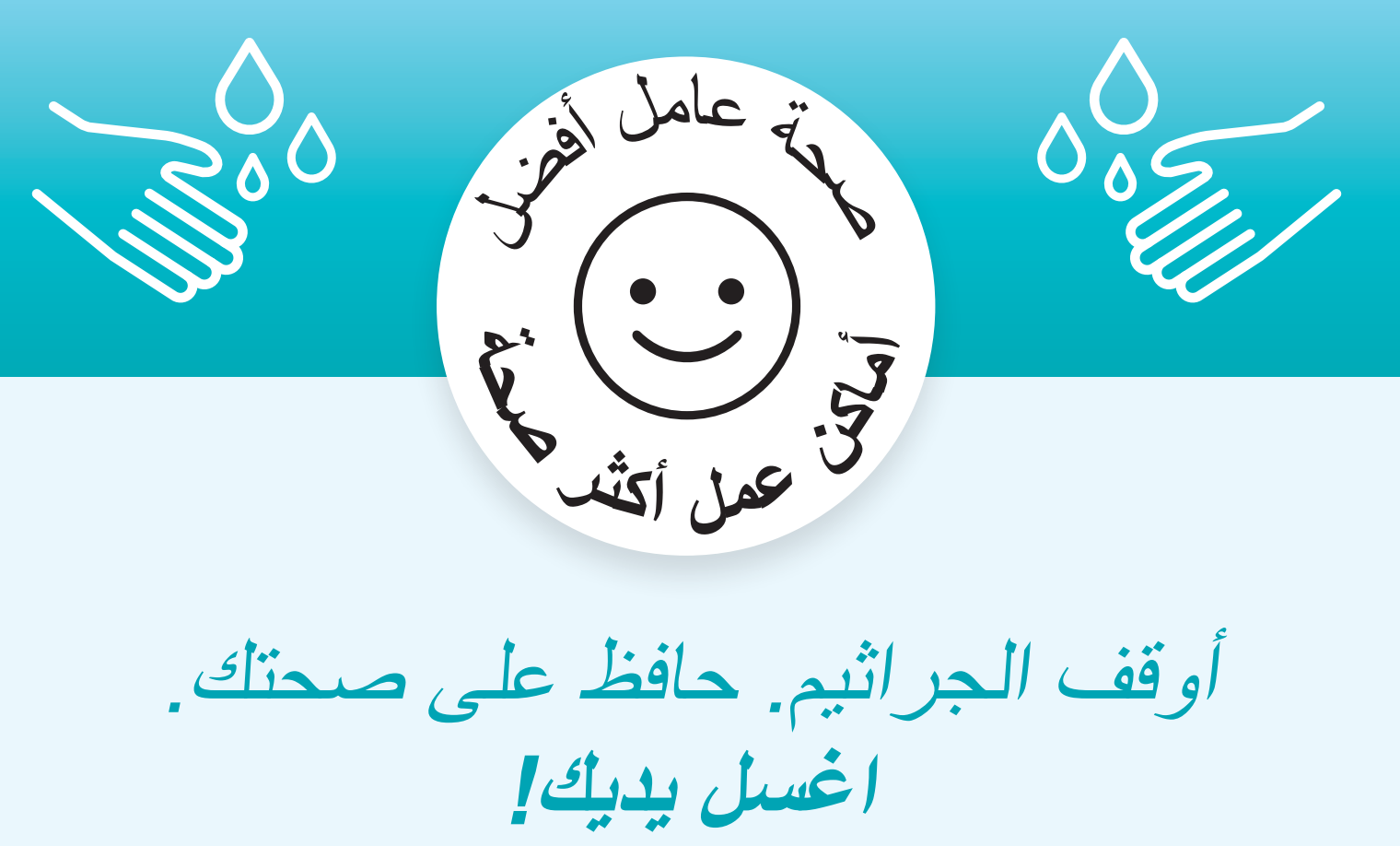

5

مدّح بجب علبلك أن تغسل بدبلك؟

(1)

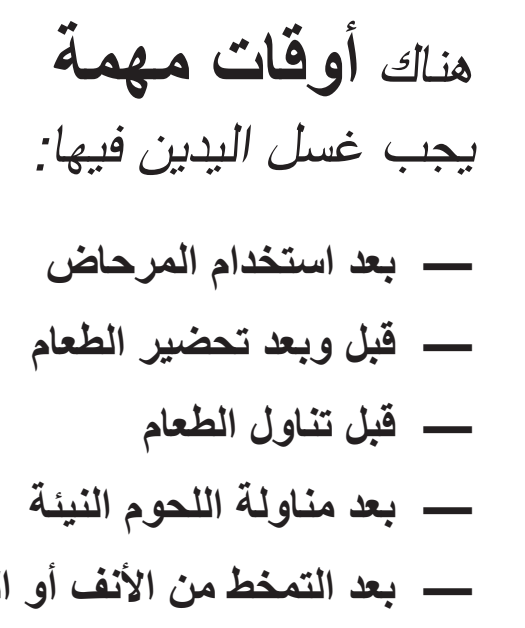

- - بعد التمخط من الأنف أو السعال أو العطاس

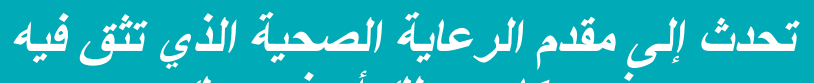

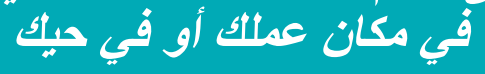



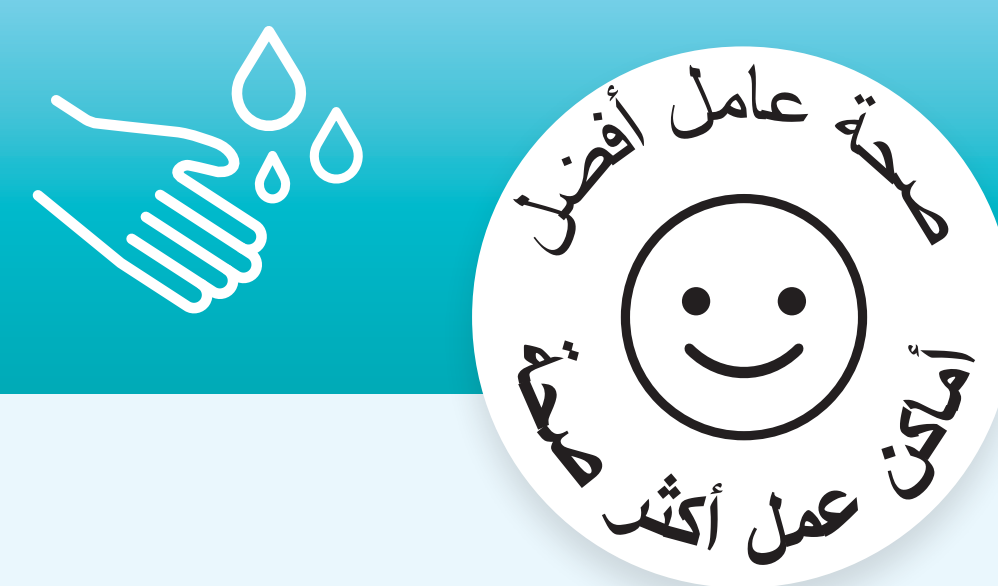

اغسل بإيكّ إل

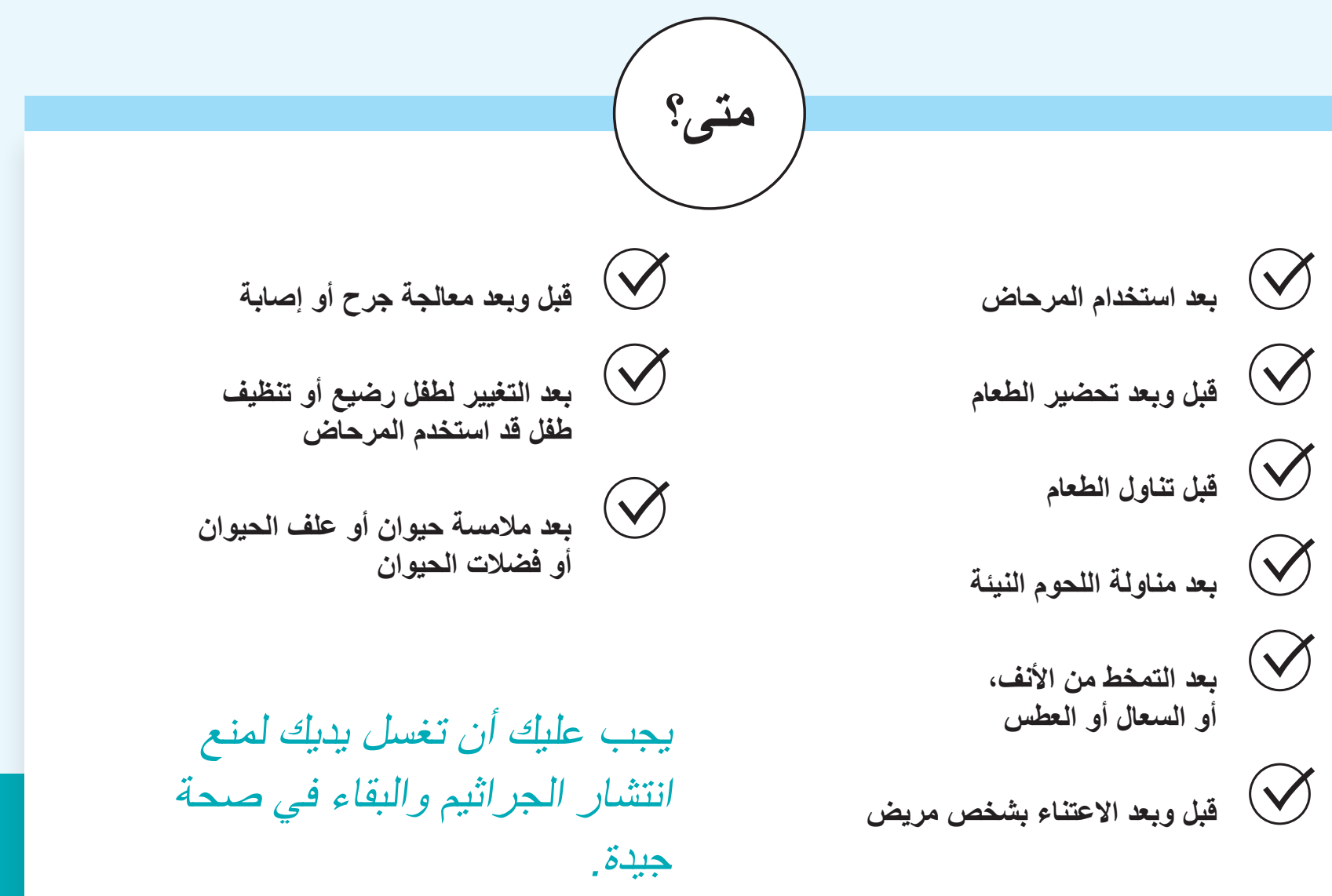

a

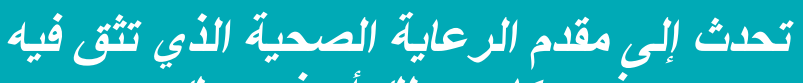

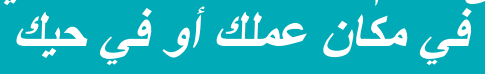



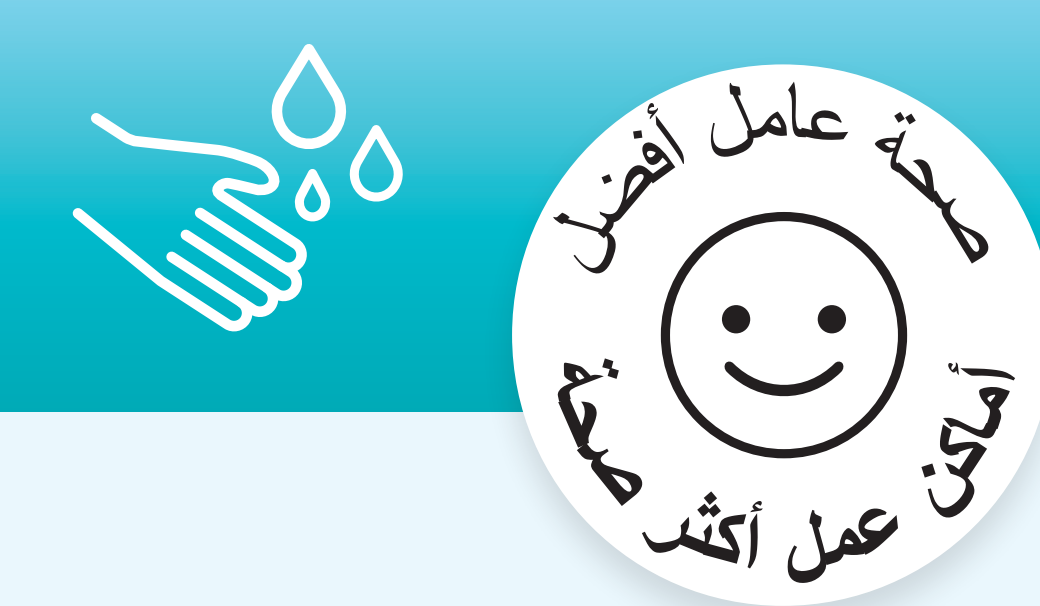

\section{أشل بإيكك إل}

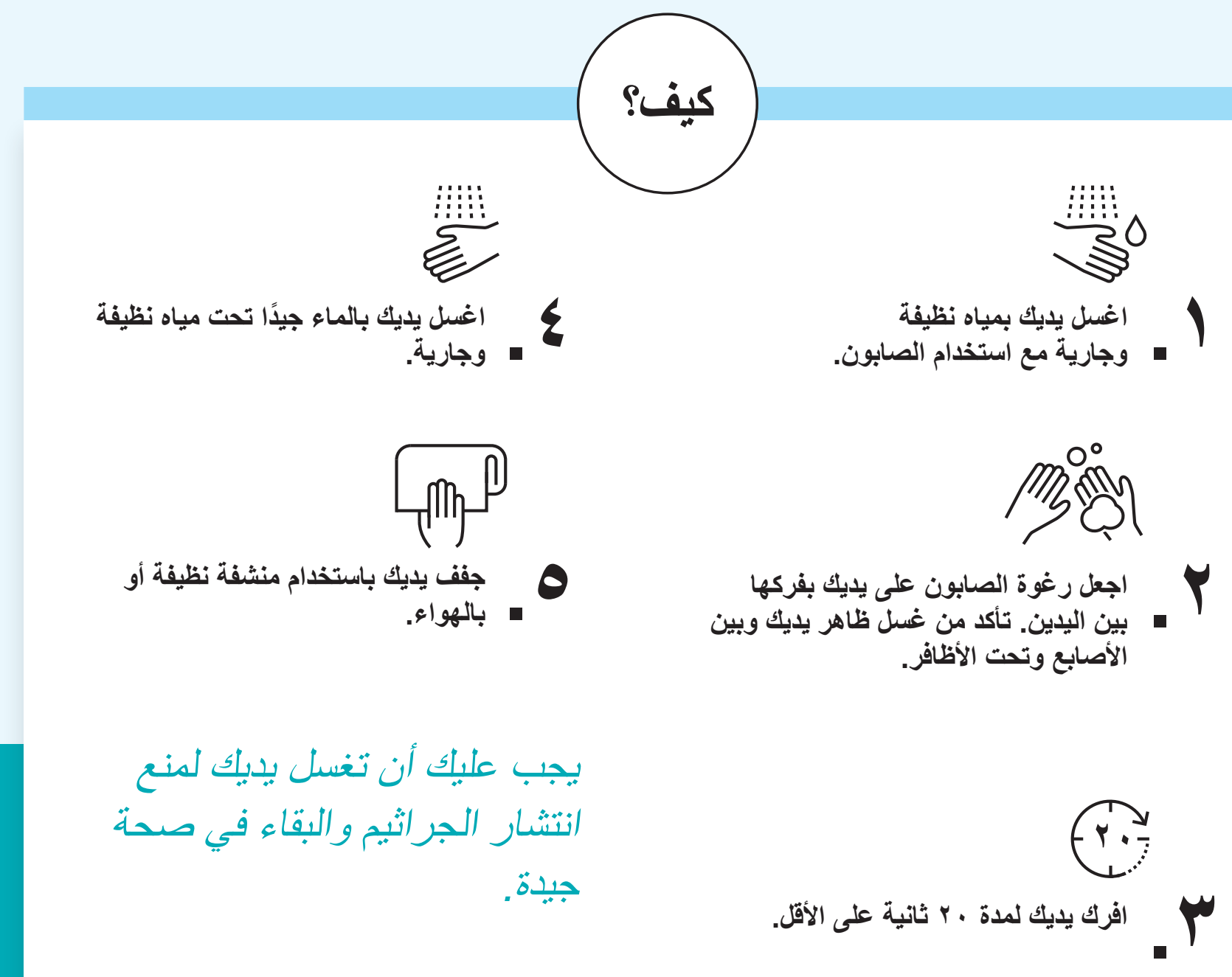

\section{a \\ تحدث إلى مثام الرعاية الصحية الذي ثثق فيه

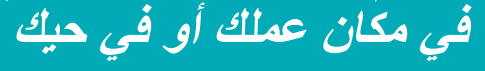




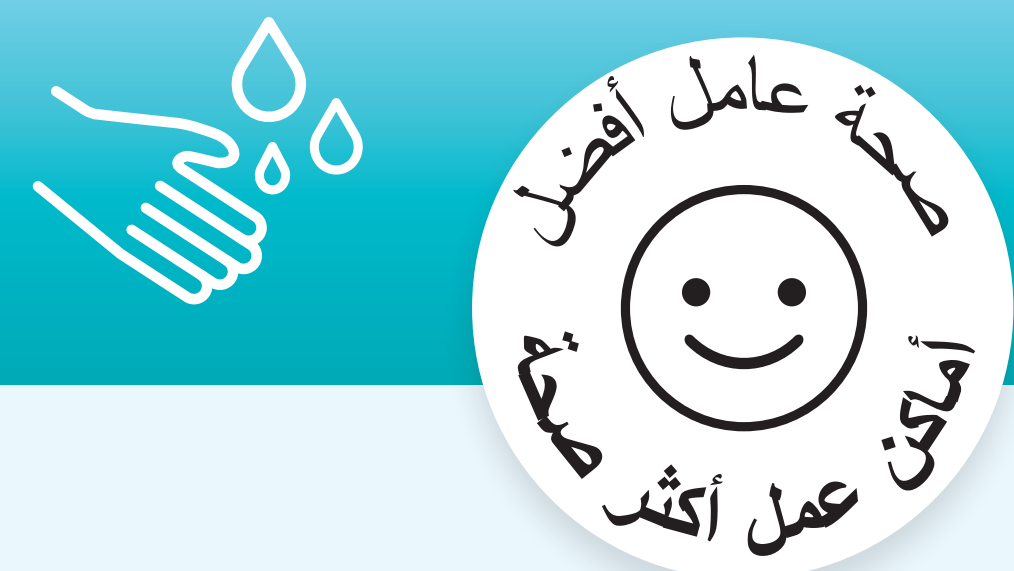

$$
\begin{aligned}
& \text { هل تعرف } \\
& \text { الحقائق التي تتعلق بغسيل الأيدي؟ }
\end{aligned}
$$

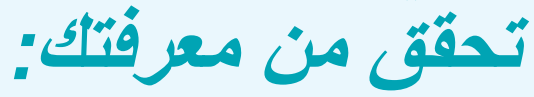

$$
\begin{aligned}
& \text { اغسل يديك بعد استخدام المرحاض مباشرة. } \\
& \text { ( اغسل يديك قبل تناول الطعام. } \\
& \text { (1) عندما تُصاب بالسعال أو العطس، تجنب غسل } \\
& \text { خواب } \\
& \text { (1) ميكن للجراثيم أن تنتشر بسبب عدم غسل }
\end{aligned}
$$

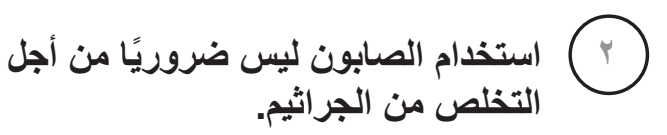

$$
\begin{aligned}
& \text { P الخطوة الأولى لغسل يديك تتمثل في ترطيب } \\
& \text { إذا لم توجد منشفة نظيفة لتجفيف يديك، فينبغي } \\
& \text { عليك تجفيفهما بالهواء. } \\
& \text { صو اب أم خطأج } \\
& \text { الإبإبـ }
\end{aligned}
$$

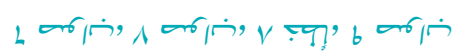

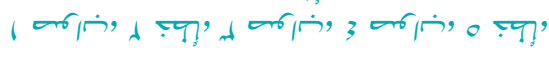

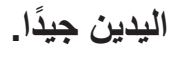

$$
\begin{aligned}
& \text { عند غسل اليدين، يجب عليك فرك يديك } \\
& \text { بالصابون جيدًا و الغسل لمدة ع ب ثنانية. } \\
& \text { عند غسل يديك، يجب عليك أن تركز فقط على بلى بلى } \\
& \text { تنظيف وغسل باطن يديك. } \\
& \text { (2) }
\end{aligned}
$$




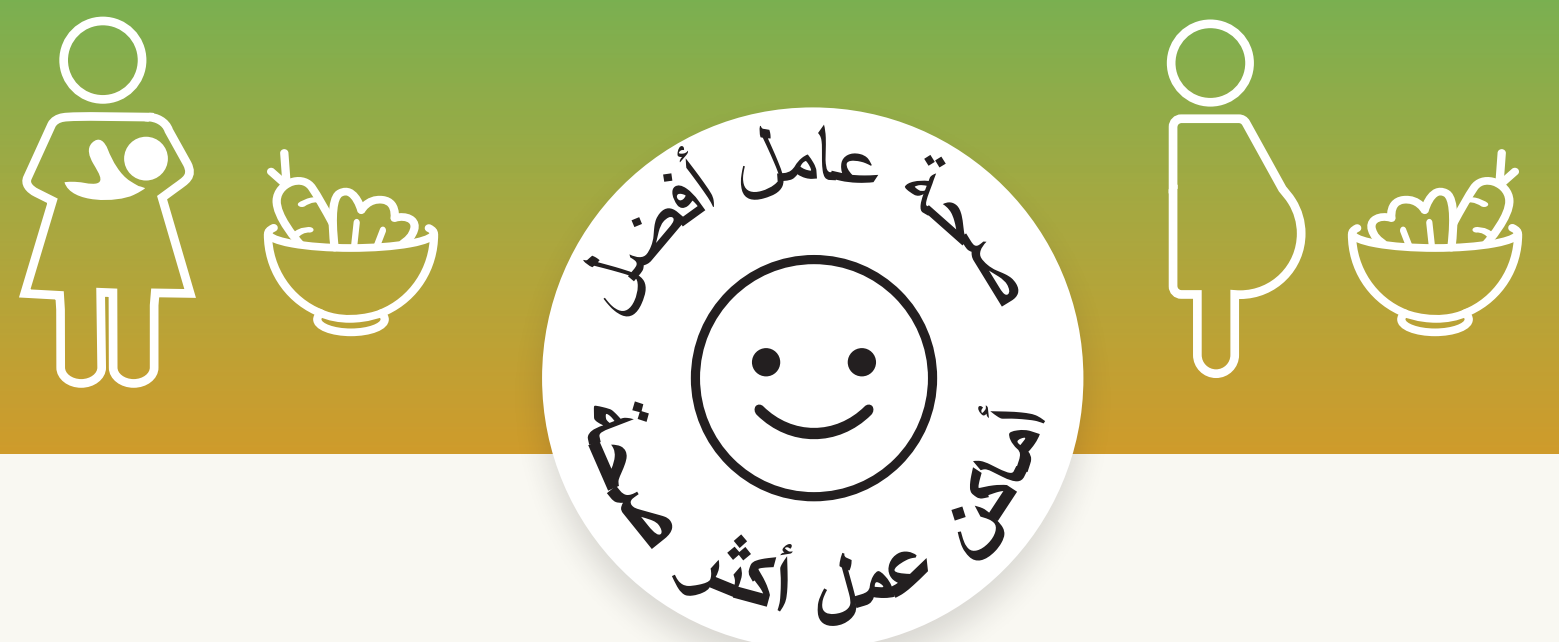

\section{(5)}

ما عادات تناول الطعام التي تساعد النساء العاملات على

الحفاظ على صحة أفضل؛

(!)

- - أناولي ثلاث وجبات يوميًا

- ـ- إذا كنتِ حبلى أو مرضعة، فتناولي وجبتين إضافيتين صغيرتين

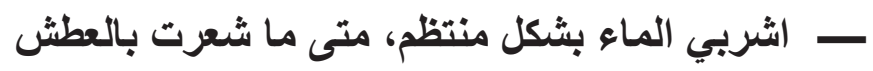

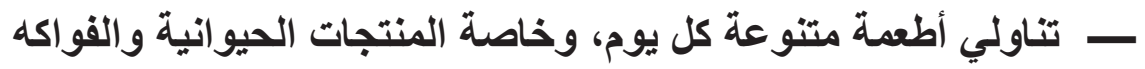

والخضر اوات

ـ - تناولي مكملات غذائية مثل الحديد وفيتامين سي للحد من الأنيميا

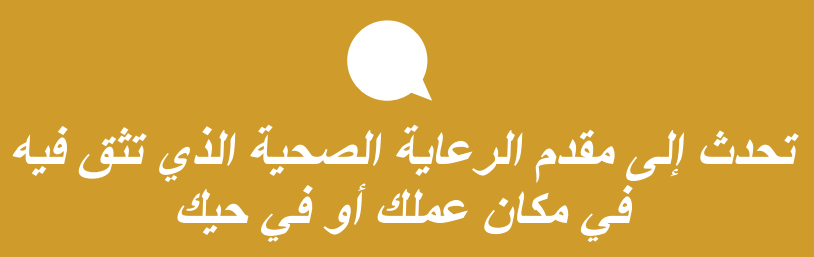



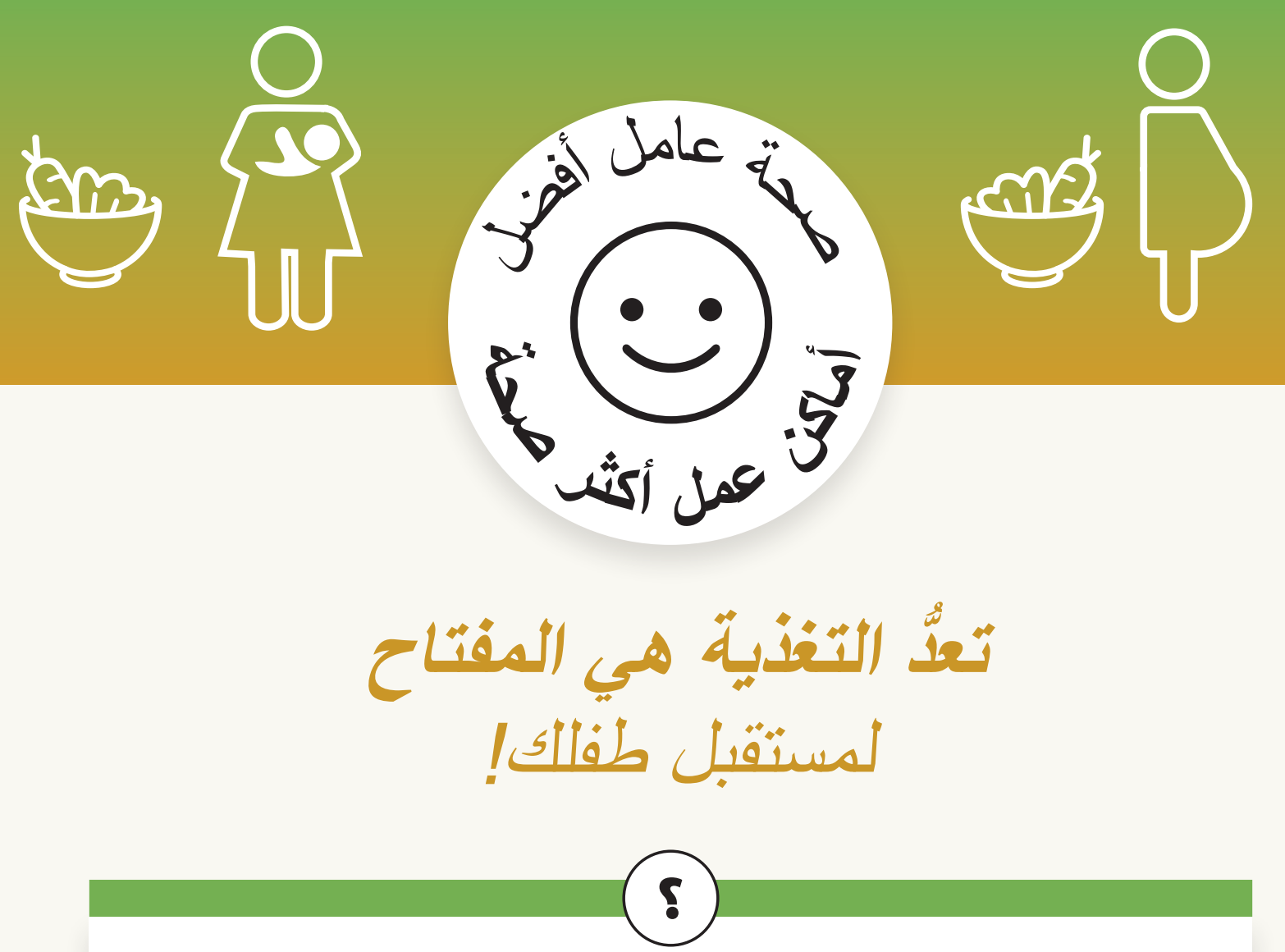

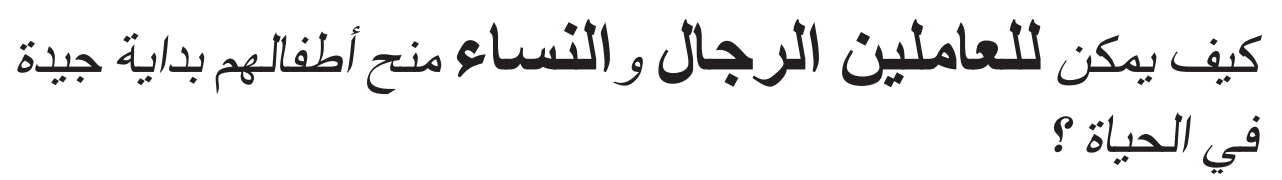

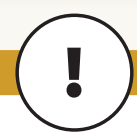

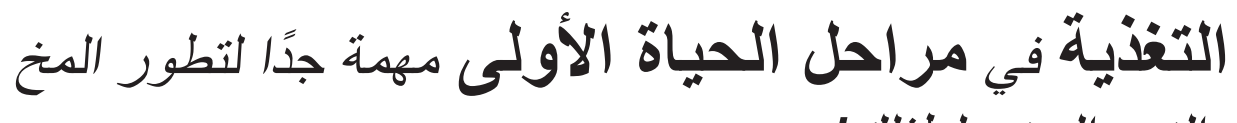
و النمو البذني لطفلك!

- اتبعي حمية غذائية صحية أثناء الحمل

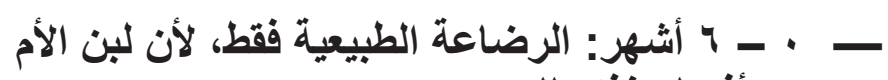
هو أفضل غذاء للرضع.

-

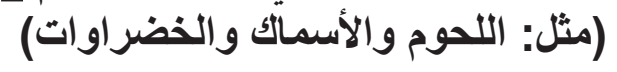

\section{Q

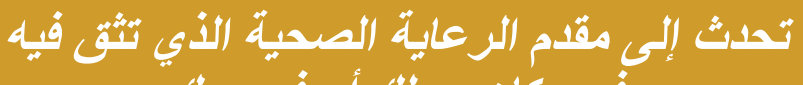

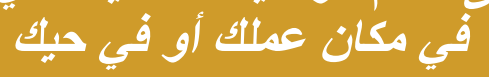




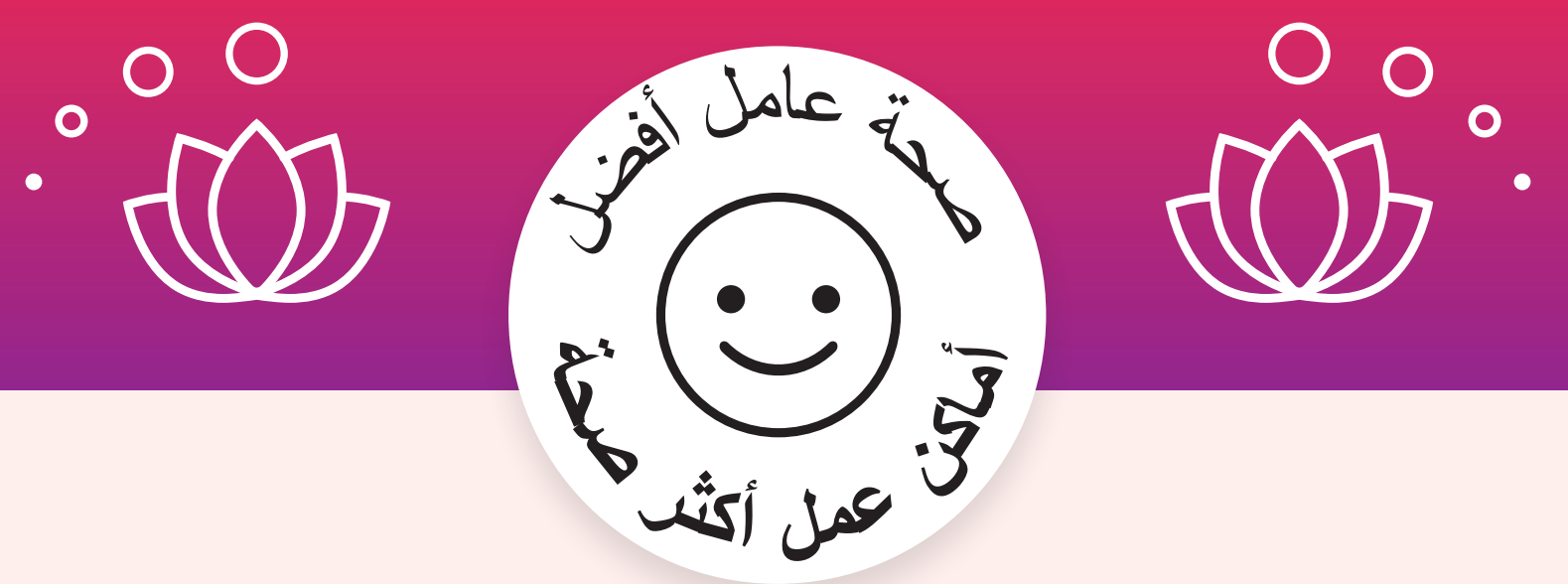

\section{حافظي على صحتّك أثناء فترة الحيض عال}

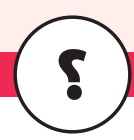

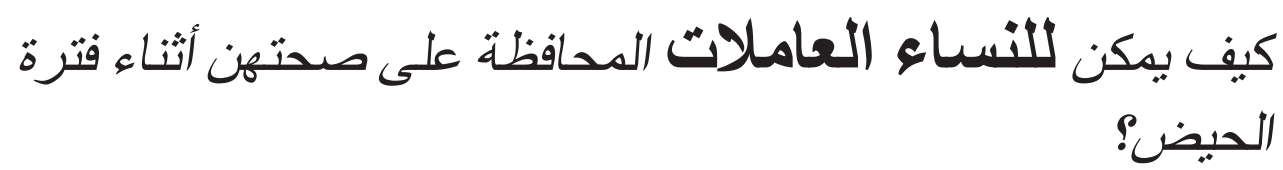

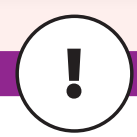

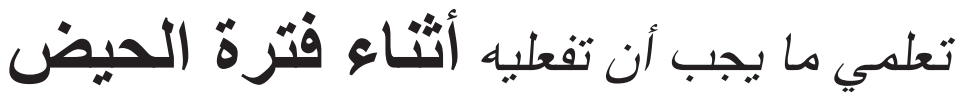
وكبف ثشعرين بتحسن:

- - استخدمي قطعة قماش نظيفة أو فوطة صحية - غيّري قطعة القماش أو الفوطة كل Y إلى Y ساعات - - خففي الألم من خلال تمارين التمدد واستخدام ضمادة دافئة أو مسكن لثلآلام - تخلصي من المخلقات بشكل مناسب - اتبعي حميتك الغذائية الصحية المعتدادة

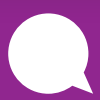

$$
\begin{aligned}
& \text { تحدث إلى مثدم الرعاية الصدية الذي ثثق فيه }
\end{aligned}
$$

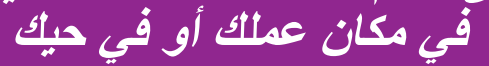




\section{.0 \\ $\bullet$

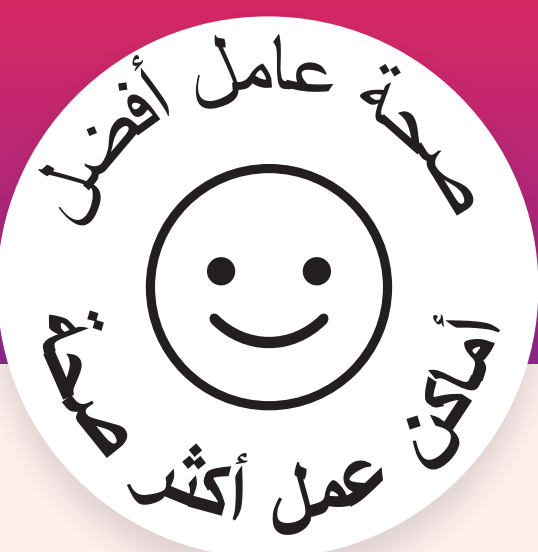 \\ $0 \circ$ \\ ○ (2)

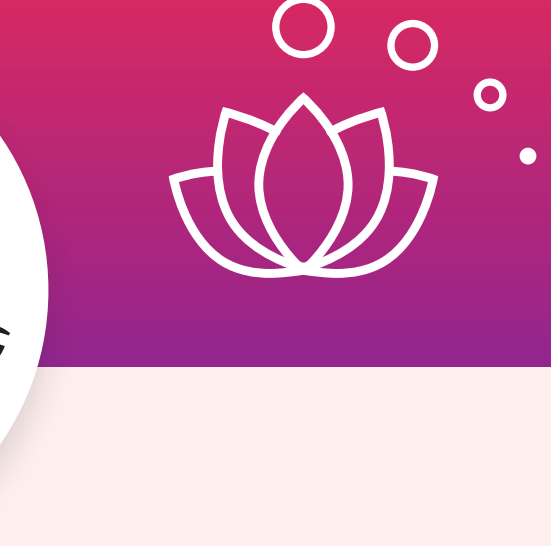 \\ حافظي على صحتك أثناء فترة الحيض.
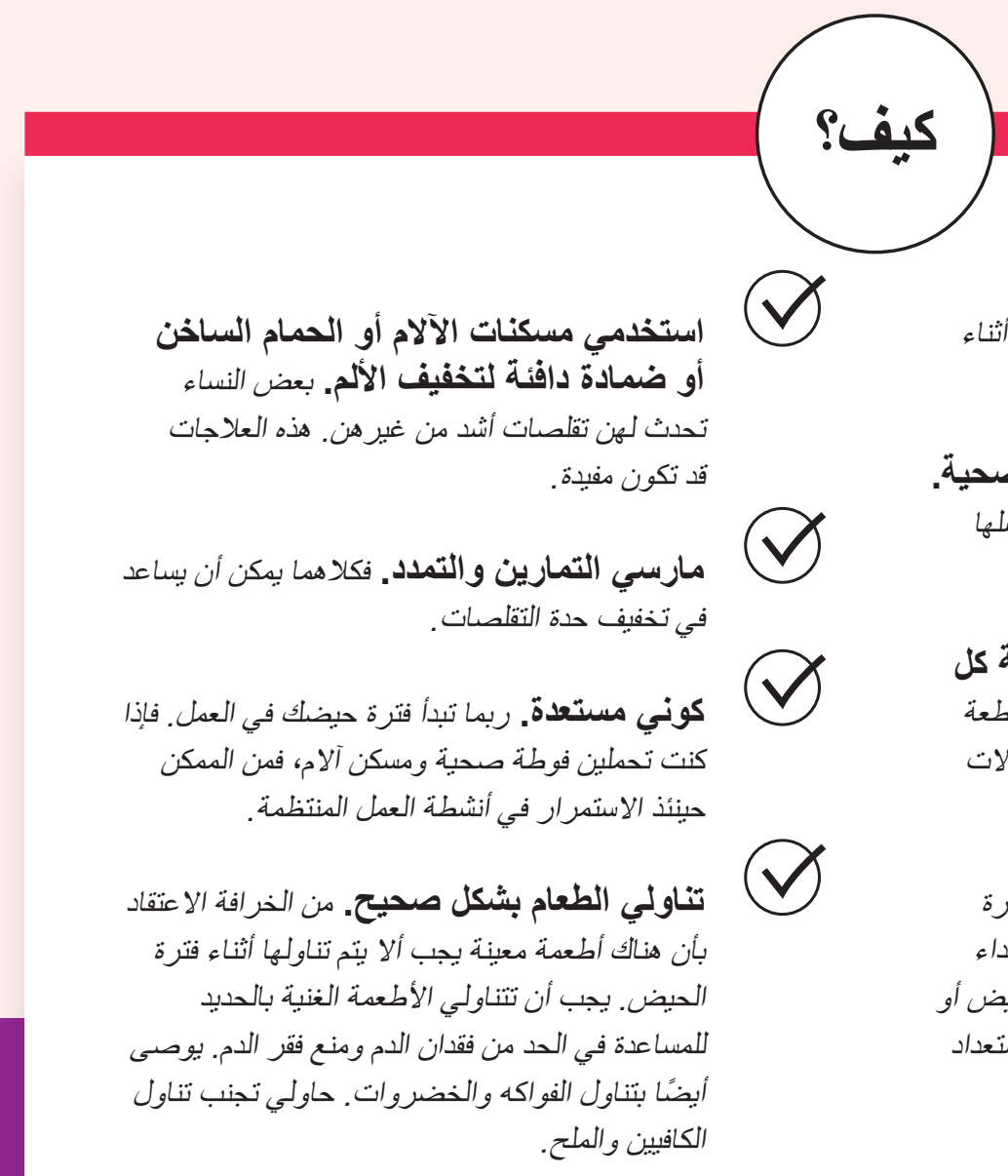 \\ حافظي على نظافتكل. استحمي بشكل بومي أثناء \\ V

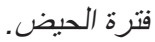 \\ استخدمي قطعة قماش نظيفة أو فوطة صحية. \\ V

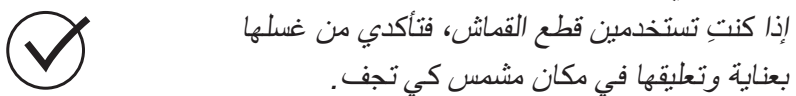 \\ V \\ V \\ غيري قطعة القماش أو الفوطة الصحية كل

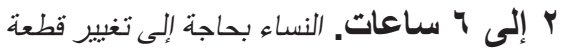 القماش أو الفوطة الصحية بشكل منتظم لهنع حالات

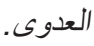 \\ V \\ ارتدي ملابس ذات ألوان غامقة. أثناء فترة

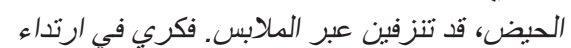

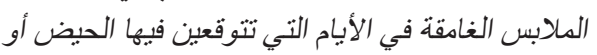

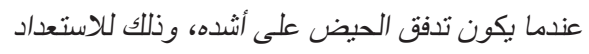

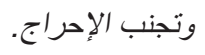

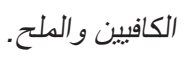




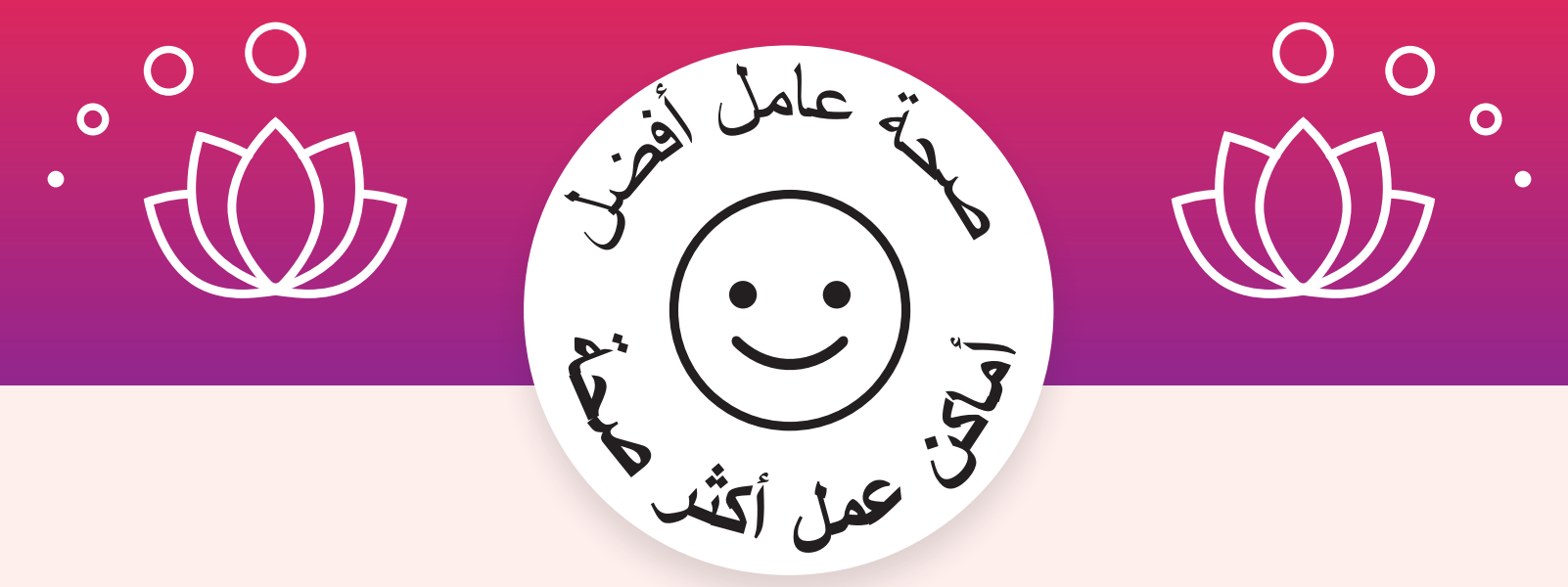

هل تعرفين الحقائق المتعلقة بالنظافة

أثناء الحبض و التعامل معه؟

تحققي من معرفتيك:

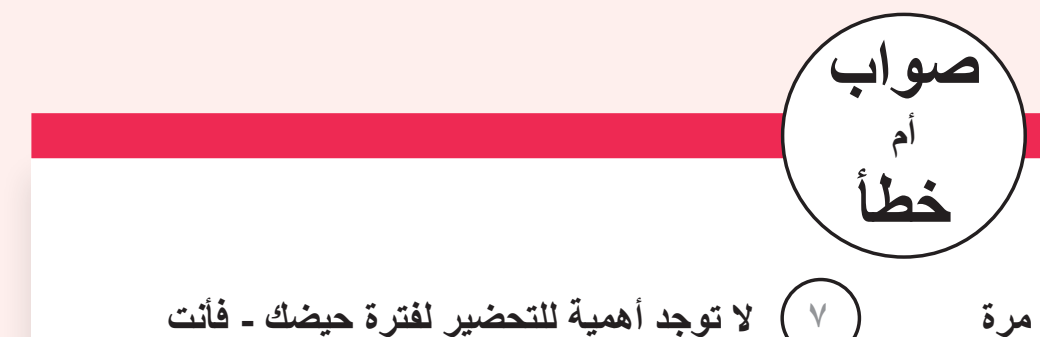

تحدث فترة الحيض لمعظم النساء حوالي مرة لا تعرفين متى ستثأتي.

واحدة في الشهر.

$1)$

فكرة أن هناك أطعمة معينة يجب عدم تناولها

أثناء الحيض تعدُ من التخاريف.

تعدُّ تقلصات المعدة من العلامات الرئيسية

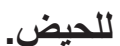

يمكن التخلص من الفوط الصحية بشكل آمن في أماكن القمامة أو يتم دفقها داخل

المرحاض.

يجب عليك الابتعاد عن أي شكل من أشكال التمارين أثناء فترة الحيض.

يجب عليك تغيير قطعة القماش أو الفوطة

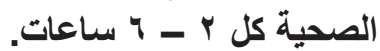

احملي بعضًا من القماش أو الفوط الصحية

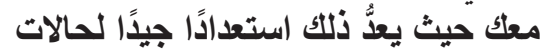

$$
\text { الإ جواباب أم خطأْ }
$$

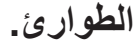

من الممكن أن تساعد مسكنات الألم أو الحمام الساخن أو الضمادة الدافئة في تخفيف الألم الألم الثمام

\section{Q \\ تحدث إلى مثام الرعاية الصحية الذي ثثق فيه في مكان عملاك أو في هيك الك}



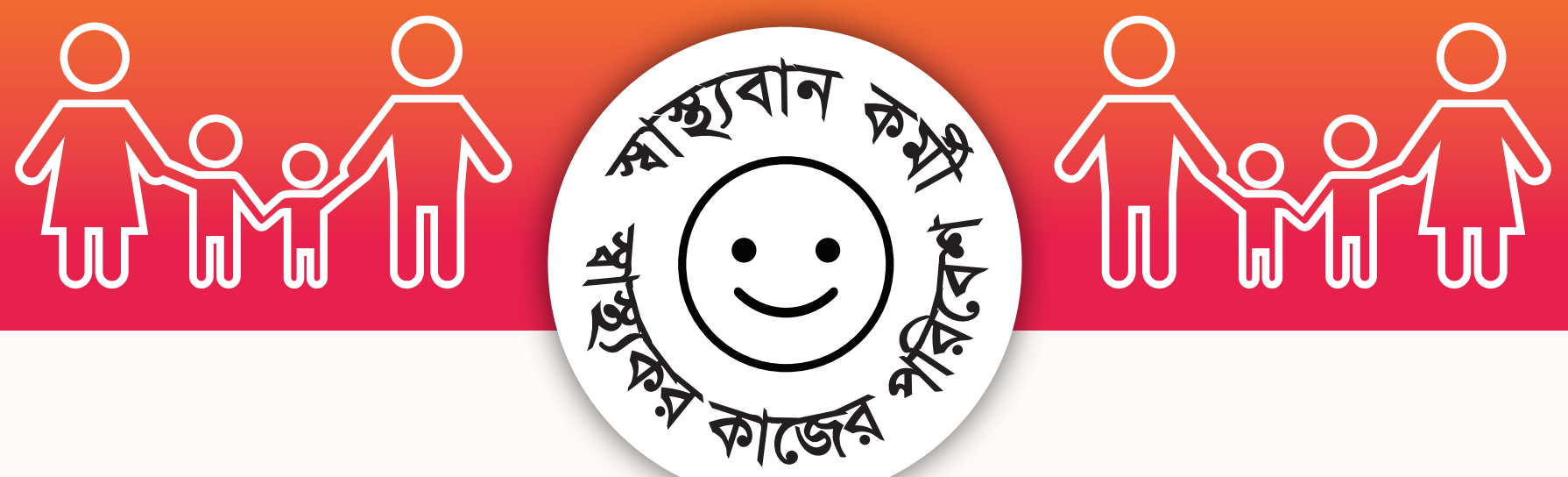

\section{পরিবার পরিকল্পনা সম্ম্পকে আপনি জানেন কি? নিজেকে যাচাই করুন:}

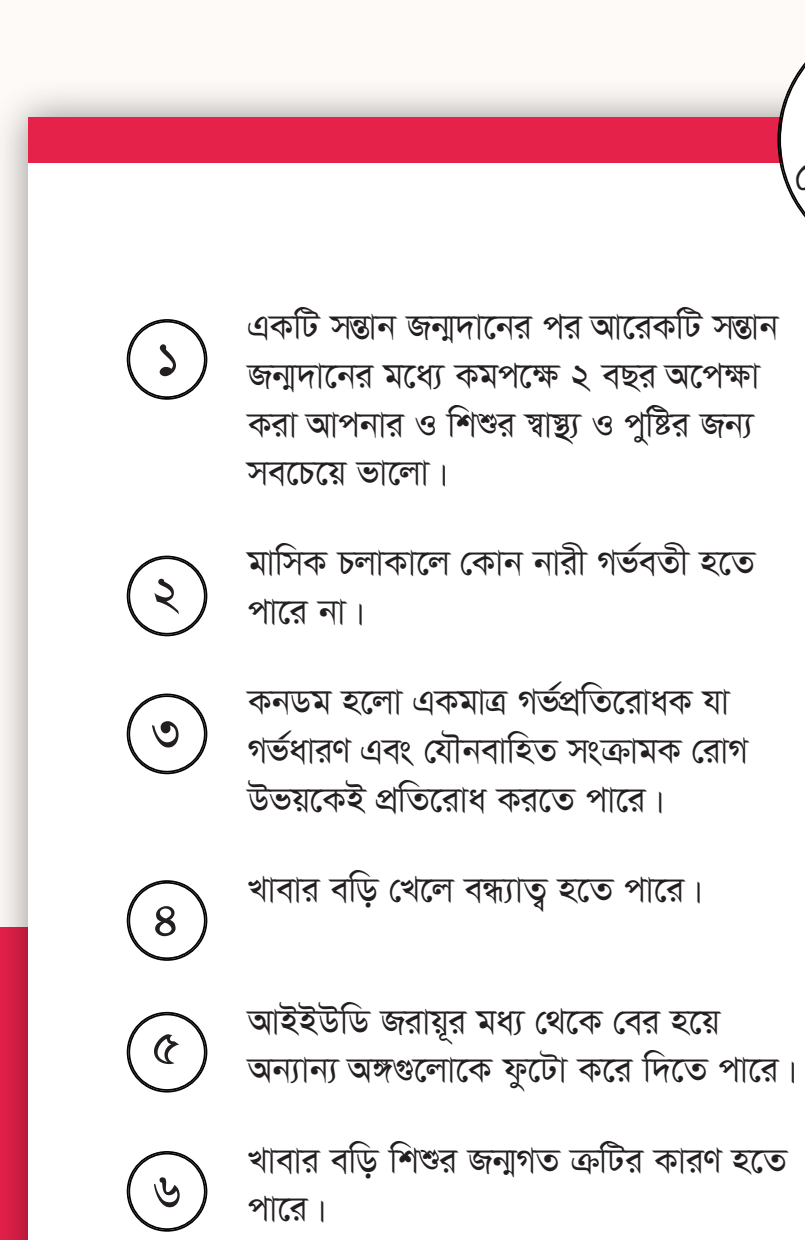

(9) ইনজেকশন মাসিকের রক্ত নিঃসরণের সাময়িক পরিবর্তন ঘটায়।

(b) শারীরিক মেলামেশার পরপরই পিল খেতে হবে

b- যা আপনাকে গর্ভধারণ থেকে বিরত রাখবে।

( শিশ্ড জন্মের প্রথম ৬ মাস শুধুমাত্র বুকের দুধ পান করালে মাকে গর্ভধারণ হতে বিরত রাখতে সহায়তা করে।

(১০) পুরুষের স্থায়ী পদ্ধতি গ্রহণের কারণে যৌন ক্ষমতা হ্রাস পায় না।

(ग) শুধুমাত্র প্রজেষ্টেরন নির্ভর খাবার বড়ি (আপন) প্রসবের ২৪ ঘন্টার মধ্যে শুরু করা যায়।

\section{উত্তরসমূহ :}

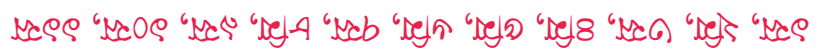

\section{আপনার কর্মইল কিংবা বসবা৫র এলাকায় কর্মরত}

ম্বাষ্ছ্য ও পরিবার কল্যাণ সবাদানকরির সাথ কথা বলুন

\section{(18)}




\section{先}

পরিবার পরিকল্পনা সর্ম্পকে আপনি জানেন কি? নিজেকে যাচাই করুন:

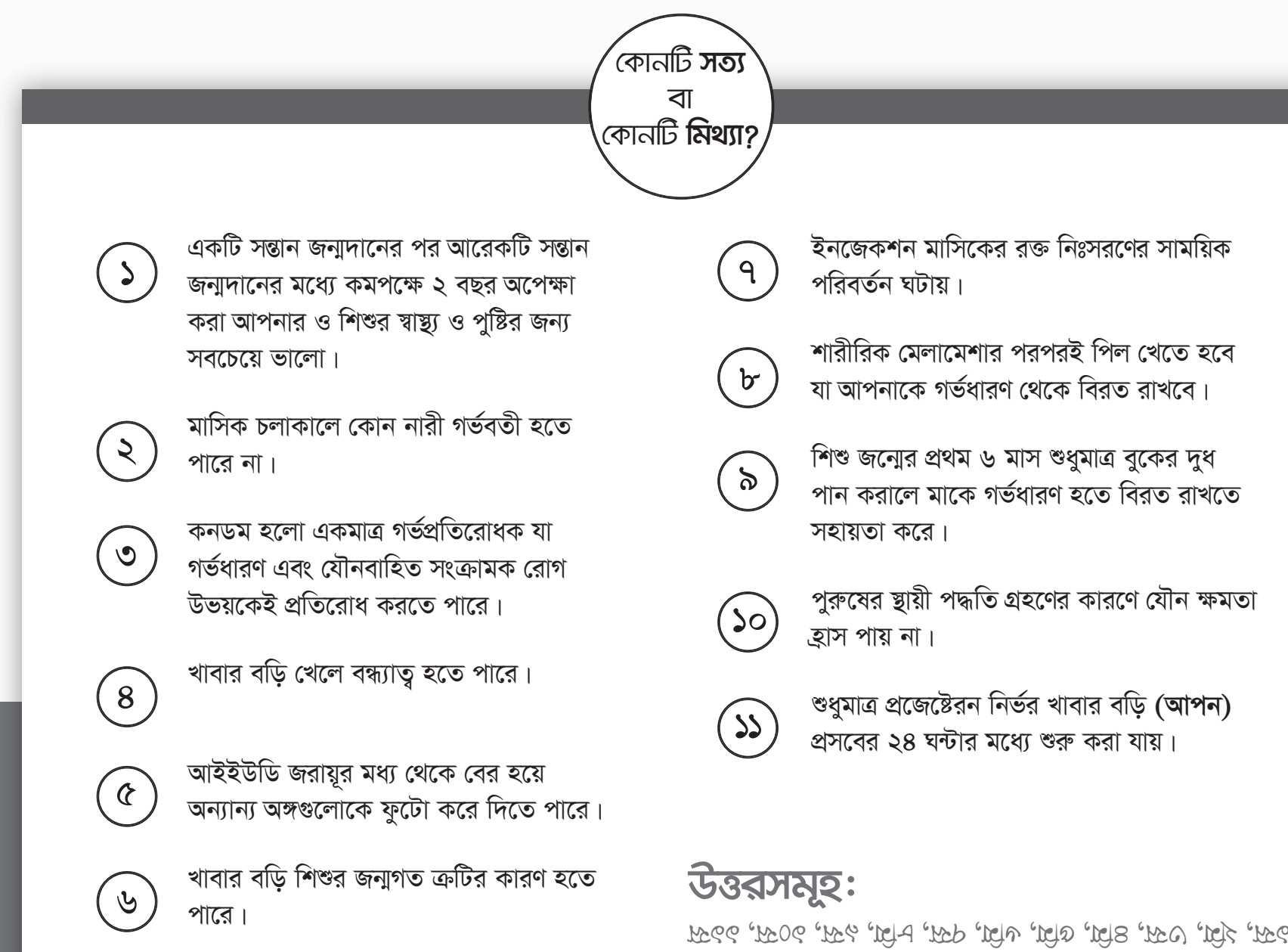

আপনার কর্মন্ল কিংবা বসবা৫র এলাকায় কর্মরত

ঘ্বাষ্ছ্য ও পরিবার কল্যাণ সেবাদানকরীর সাথে কথা বলুন 


\section{অত্যন্ত কার্যকর}

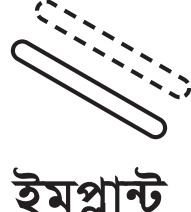

ইমপ্লান্ট

৩-৫ বছর

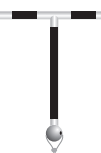

আইইউডি

১০ বছর

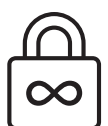

বন্ধ্যাকরণ

স্থায়ী
কার্যকর

কম কার্যকর

$\because 000000000$

স্বল্প মাত্রার খাবার

বড়ি (আপন)

থ्रতিদিন

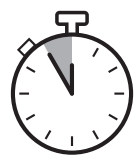

আজল

প্রতিবার

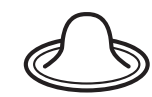

পুরুষ্ব কনডম

ওধুমাত্র ১ বার

ব্যবহার করা যায়

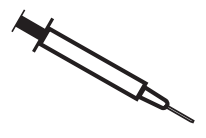

ইনজেকশন

(ডিপোপ্রোভেরা)

৩ মাস

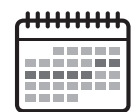

প্রাকৃতিক দিন গোনা পদ্ধতি

श्रতিদিन
আপনার কর্মন্মুল কিংবা বসবালের এলাকায় কর্মরত

ত্বান্য্য ও পরিবার কল্যাণ সেবাদানকারীর সাথে কথা বলুন

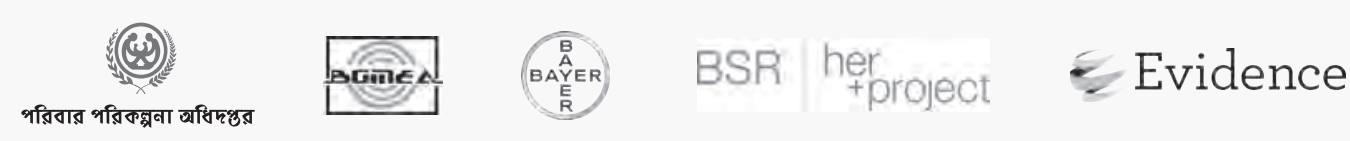


(1) একটি সুস্থ সন্তান, মা ও স্বাস্থ্যবান পরিবার নিশ্চিত করার সবচেয়ে ভালো উপায় হল সন্তানদের মধ্যে বয়সের ব্যবধান রাখা। এই ব্যবধান মায়েদেরকে প্রসব থেকে সেরে উঠতে ও বাবাদেরকে তাদের সন্তানদের জন্য পর্যাপ্ত অর্থনৈতিক স্বচ্ছলতা ও সময় পাওয়ার সুযোগ তৈরী করে. দেয়। বয়সের ব্যবধানের জন্য একটি আধুনিক পরিবার পরিকল্পনা পদ্ধতি ব্যবহার করুন এবং পুনরায় গর্ভধারণ করতে চাইলে সর্বশেষ সন্তানের বয়স কমপক্ষে ২ বছর না হওয়া পর্যন্ত অপেক্ষা করুন।

(!) খাবার বড়ি শিষ্র জন্মগত ক্রটি ঘটায় না বা ভ্ণণণর ক্ষতি করে না, যদি আপনি বড়ি চলাকালীন সময়ে গর্ভবতী হয়ে পড়েন অথবা ভূলবশত গর্ভবতী হয়ে পড়ার পরে বড়ি খাওয়া ওুরু করেন।

(1) কনডম হলো একমাত্র গর্ভপ্রতিরোধক যা গর্ভধারণ এবং এইচআইভি সহ যৌনবাহিত সংক্রামক রোগ উভয়কে প্রতিরোধ করে। কনডমের মধ্যে দিল্যে কোন কিছুই ভেদ করে বেরোতে পারে না, যতক্ষণ ना এটি ছিঁড়ে याয়।
(!) পরিবার পরিকল্পনার পদ্ধতিগুলো পরবর্তিতে সন্তান ধারনের অক্ষমতা সৃষ্টি করে না। আপনার শরীর আবার গর্ভবতী হওয়ার অবস্থয় ফিরে আসতে কয়েক মাস সময় লাগতে পারে। এটি সাময়িক। কোন সুম্থ নারী যতদিন ধরেই হরমোনাল পরিবার পরিকল্পনা পদ্ধতি ব্যবহার করুক না কেন গর্ভধারণ ক্ষমতা বা উর্বরতা আবার ফিরে আসে।

(1) আইইউডি একটি অত্যন্ত কার্যকর পদ্ধতি যা ১০ বছর পর্যত্ত গর্ভাধারণ রোধ করতে পারে। কোন প্রশিক্ষিত ব্যক্তির দ্বারা সঠিকভাবে আইইউডি পরানো হনে এটি যথাস্থানে থাকে। এটি কখনও জরায়ূ থেকে শরীরের অন্যান্য অংশে সরে যেতে পারে না।

(!) আধুনিক গর্ভনিরোধকগুলো সহজেই সহনশীল এবং অত্যন্ত বিশ্বাসয়োগ্য। এমনকি এগুনো আপনাকে কিছু ধরণের ক্যান্সারের হাত থেকে রক্ষা করে যেমন জরায় ও ডিম্বাশয়ের ক্যান্সার। ইমপ্লান্ট আপনাকে তলপেটের প্রদাহ থেকে সুরক্ষা প্রদান করে। ওধুমাত্র 80 উর্দ্ধ নারী যারা ধূমপান করেন তাদেরকে মিশ্র খাবার বড়ি এড়িয়ে চলার পরামর্শ দেওয়া যেতে পারে।
(!) গর্ভধারণরোধ করতে খাবার বড়ি প্রতিদিন অবশ্যই ধারাবাহিকভাবে খেতে হবে। মনে রাখতে হবে শুধুমাত্র মিলনের পর বড়ি খেয়ে গর্ভপ্রতিরোধ নিশ্চিত করা যায় না। यদি একদিন বড়ি খেতে ভুলে যান কিংবা মাসিক শুরুর পাঁচ দিন পর থেকে প্যাকেটের বড়ি খাওয়া শুরু করেন, তাহলে আপনাকে অবশ্যই অন্ততপক্ষে পরবর্তী সাতদিন অন্য একটা সহায়ক পদ্ধতি কনডম ব্যবহার করতে হবে। জরুরী গর্ভপ্রতিরোধক বা ইমার্জেন্সি পিল পাওয়া যায়, এগুনো আপনার নিয়মিত বড়িগুলো থেকে বেশি শক্তিশালী বনে শু ধুমাত্র জরুরি থ্রয়োজনে ব্যবহার করা উচিত। কখনই বেশি বেশি ব্যবহার করা উচিতি নয়।

(!) শিঙ্তকে ৬ মাস পর্যন্ত ঞধুমাত্র বুকের দুধ পান করান। এই সময় তাকে বাড়তি এক ফেঁঁটা পানিও দিবেন না। মায়ের বুকের দুধ শিঞ্তের শারীরিক ও মানসিক বিকাশ এবং রোগ প্রতিরোধ ক্ষমতা নিশ্চিত করে। পরবর্তী সন্তান পর্যাপ্ত বিরতিতে নেয়া নিশ্চিত করতে আপনার জন্য উপযোগী যেকোন একটি পরিবার পরিকল্পনা পদ্ধতি যেমন: কনডম, খাবার বড়ি, ইননজেকশন, আইইউডি, ইমপ্ল্যান্ট ব্যবহার করুন
(1) মাসিক চলাকালে আপনি গর্ভবতী হতে পারেন। । यদি আপনি কোন প্রাকৃতিক পরিবার পরিকল্পনা পদ্ধতি (দিন গোনা) ব্যবহার করেন, তাহলে আপনি অবশ্যই মজো।্যোগ সহকারে আপনার মাসিকের দিন-তারিখ মনে রাখবেন এবং কখনই মনে করবেন না য্যে মাসিক চলাকালে আপনার গর্ভবতী হওয়ার সম্ভাবনা নাই।

(1) ইনজেকশন অত্যন্ত কার্যকর স্বল্পস্থায়ী পদ্ধতি যা প্রতি ৩ মাস অন্তর একটি করে নেওয়ার প্রয়োজন হয়। আপনার হয়जে অনিয়মিত রক্তপাত, ভারী রক্তপাত অথবा খুব অল্প সময়ের জন্য দীর্ঘशয়য় রক্তপাত দেখা দিতে পারে। খুব কম নারীর মধ্যে মাথা ব্যথা, মাথা ঘোরা, স্তনে চিড়িিড়ে ব্যথা এবং ওজন বেড়ে যাওয়ার প্রবণতা দেখা যায়। এগুলো অসুসৃতার লক্ষণ নয়। ইনজেকশন ব্যবহারের প্রথম কয়েক মালের মধ্যে সাধারণত এই সমস্যাগুলি চলে যায়।
আপনার কর্মন্ছুল কিংবা বসবালের এলাকায় কর্মরত স্বান্য্য ও পরিবার কল্যাণ সেবাদানকারীর সাথে কথা বলুন
(48) পরিবার পরিকল্পনা অধिদদপ্তর

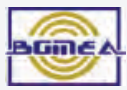


একটি সুস্থ সন্তান, মা ও স্বাস্থ্যবান পরিবার নিশ্চিত করার সবচেয়ে ভালো উপায় হল সন্তানদের মধ্যে বয়সের ব্যবধান রাখা। এই ব্যবধান মায়েদেরকে প্রসব থেকে সেরে উঠতে ও বাবাদেরকে তাদের সন্তানদের জন্য পর্যাপ্ত অর্থনৈতিক স্বচ্ছলতা ও সময় পাওয়ার সুযোগ তৈরী করে. দেয়। বয়সের ব্যবধানের জন্য একটি আধুনিক পরিবার পরিকল্পনা পদ্ধতি ব্যবহার করুন এবং পুনরায় গর্ভধারণ করতে চাইলে সর্বশেষ সন্তানের বয়স কমপক্ষে ২ বছর না হওয়া পর্যন্ত অপেক্ষা করুন।

(!) খাবার বড়ি শিশ্রর জন্মগত ক্রটি ঘটায় না বা ভ্রুণের ক্ষতি করে না, যদি আপনি বড়ি চলাকালীন সময়ে গর্ভবতী হয়ে পড়েন অথবা ভূলবশত গর্ভবতী হয়ে পড়ার পরে বড়ি খাওয়া ওরু করেন।

(1) কনডম হলো একমাত্র গর্ভপ্রতিরোধক যা গর্ভধারণ এবং এইচআইভি সহ যৌনবাহিত সংক্রামক রোগ উভয়কে প্রতিরোধ করে। কনডমের মধ্যে দিল্যে কোন কিছুই ভেদ করে বেরোতে পারে না, যতক্ষণ ना এটि ছিঁড়ে याয়।
(!) পরিবার পরিকল্পনার পদ্ধতিগুলো পরবর্তিতে সন্তান ধারনের অক্ষমতা সৃষ্টি করে না। আপনার শরীর আবার গর্ভবতী হওয়ার অবস্থয় ফিরে আসতে কয়েক মাস সময় লাগতে পারে। এটি সাময়িক। কোন সুষ্থ নারী যতদিন ধরেই হরমোনাল পরিবার পরিকল্পনা পদ্ধতি ব্যবহার করুক না কেন গর্ভধারণ ক্ষমতা বা উর্বরতা আবার ফিরে আসে।

(1) আইইউডি একটি অত্যন্ত কার্যকর পদ্ধতি যা ১০ বছর পর্যত্ত গর্ভাধারণ রোধ করতে পারে। কোন প্রশিক্ষিত ব্যক্তির দ্বারা সঠিকভাবে আইইউডি পরানো হনে এটি যথাস্থানে থাকে। এটি কখনও জরায়ূ থেকে শরীরের অন্যান্য অংশে সরে যেতে পারে না।

(!) আধুনিক গর্ভনিরোধকগুলো সহজেই সহনশীল এবং অত্যন্ত বিশ্বাসয়োগ্য। এমনকি এগুনো আপনাকে কিছু ধরণের ক্যান্সারের হাত থেকে রক্ষা করে যেমন জরায়ূ ও ডিম্বাশয়ের ক্যান্সার। ইমপ্লান্ট আপনাকে তলপেটের প্রদাহ থেকে সুরক্ষা প্রদান করে। ওধুমাত্র 80 উর্দ্ধ নারী যারা ধূমপান করেন তাদেরকে মিশ্র খাবার বড়ি এড়িয়ে চলার পরামর্শ দেওয়া যেতে পারে।
(!) গর্ভধারণরোধ করতে খাবার বড়ি প্রতিদিন অবশ্যই ধারাবাহিকভাবে খেতে হবে। মনে রাখতে হবে শুধুমাত্র মিলনের পর বড়ি খেয়ে গর্ভপ্রতিরোধ নিশ্চিত করা যায় না। यদি একদিন বড়ি খেতে ভুলে যান কিংবা মাসিক শুরুর পাঁচ দিন পর থেকে. প্যাকেটের বড়ি খাওয়া শুরু করেন, তাহুে আপনাকে অবশ্যই অন্ততপক্ষে পরবর্তী সাতদিন অন্য একটা সহায়ক পদ্ধতি কনডম ব্যবহার করতে হবে। জরুরী গর্ভপ্রতিরোধক বা ইমার্জেন্সি পিল পাওয়া যায়, এগুনো আপনার নিয়মিত বড়িগুলো থেকে বেশি শক্তিশালী বলে শুধুমাত্র জরুরি প্রয়োজনে ব্যবহার করা উচিত। কখনই বেশি বেশি ব্যবহার করা উচিতি নয়।

(!) শিঙ্তকে ৬ মাস পর্যন্ত ঞধুমাত্র বুকের দুধ পান করান। এই সময় তাকে বাড়তি এক ফেঁঁটা পানিও দিবেন না। মায়ের বুকের দুধ শিঞ্তের শারীরিক ও মানসিক বিকাশ এবং রোগ প্রতিরোধ ক্ষমতা নিশ্চিত করে। পরবর্তী সন্তান পর্যাপ্ত বিরতিতে নেয়া নিশ্চিত করতে আপনার জন্য উপযোগী যেকোন একটি পরিবার পরিকল্পনা পদ্ধতি যেমন: কনডম, খাবার বড়ি, ইনজজেশন, আইইউডি, ইমপ্য্যান্ট ব্যবহার করুন
(1) মাসিক চলাকালে আপনি গর্ভবতী হতে পারেন। য यি আপনি কোন খ্রাকৃতিক পরিবার পরিকল্পনা পদ্ধতি (দিন গোনা) ব্যবহার করেন, তাহলে আপনি অবশ্যই মজো।্যোগ সহকারে আপনার মাসিকের দিন-তারিখ মনে রাখবেন এবং কখনই মনে করবেন না যে মাসিক চলাকালে আপনার গর্ভবতী হওয়ার সম্ভাবনা নাই।

(1) ইনজেকশন অত্যন্ত কার্যকর স্বল্পস্থায়ী পদ্ধতি যা প্রতি ৩ মাস অন্তর একটি করে নেওয়ার প্রয়োজন হয়। আপনার হয়जে অনিয়মিত রক্তপাত, ভারী রক্তপাত অথবा খুব অল্প সময়ের জন্য দীর্ঘशয়য় রক্তপাত দেখা দিতে পারে। খুব কম নারীর মধ্যে মাথা ব্যথা, মাথা ঘোরা, স্তন চিড়চিড়় ব্যথা এবং ওজন বেড়ে যাওয়ার থ্রবণতা দেখা যায়। এগুনো অসুशৃতার লঙ্ষণ নয়। ইনজেকশন ব্যবহারের প্রথম কয়েক মালের মধ্যে সাধারণতত এই সমস্যাঞলি চলে याয়। 

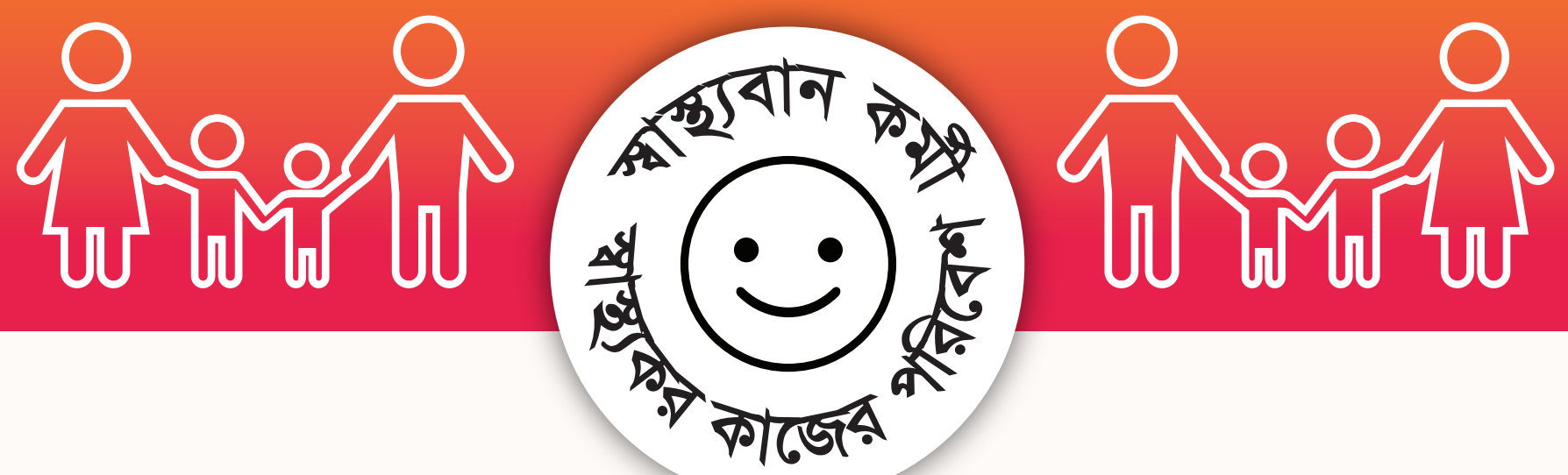

\section{পরিবার পরিকল্পনা কোন সুযোগ নয় পছন্দমত পদ্ধতি গ্রহণ করা!}

\section{$?$}

আপনার জন্য সব৫য়ে কার্যকর উপায় কী হতে পারে? যদি আপনি ;

- পরিকল্পিত পরিবার গঠন করতে চান

- অনাকাজ্ষিত গর্ভধারণ প্রতিরোধ করতে চান

- অনিরাপদ গর্ভপাত এড়াতে চান

\section{!}

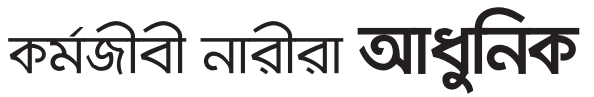

পরিবার পরিকল্পনা পদ্ধতি ব্যবহার করেন!

0000000000

খাবার বড়ি

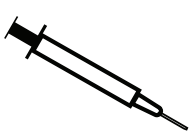

ইনজেকশন

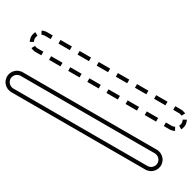

ইমপ্লান্ট

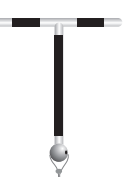

আইইউডি

আপনার কর্মন্ইল কিংবা বসবালর এলাকায় কর্মরত

স্বান্ছ্য ও পরিবার কল্যাণ লবাদানকারীর সাথে কথা বলুন 


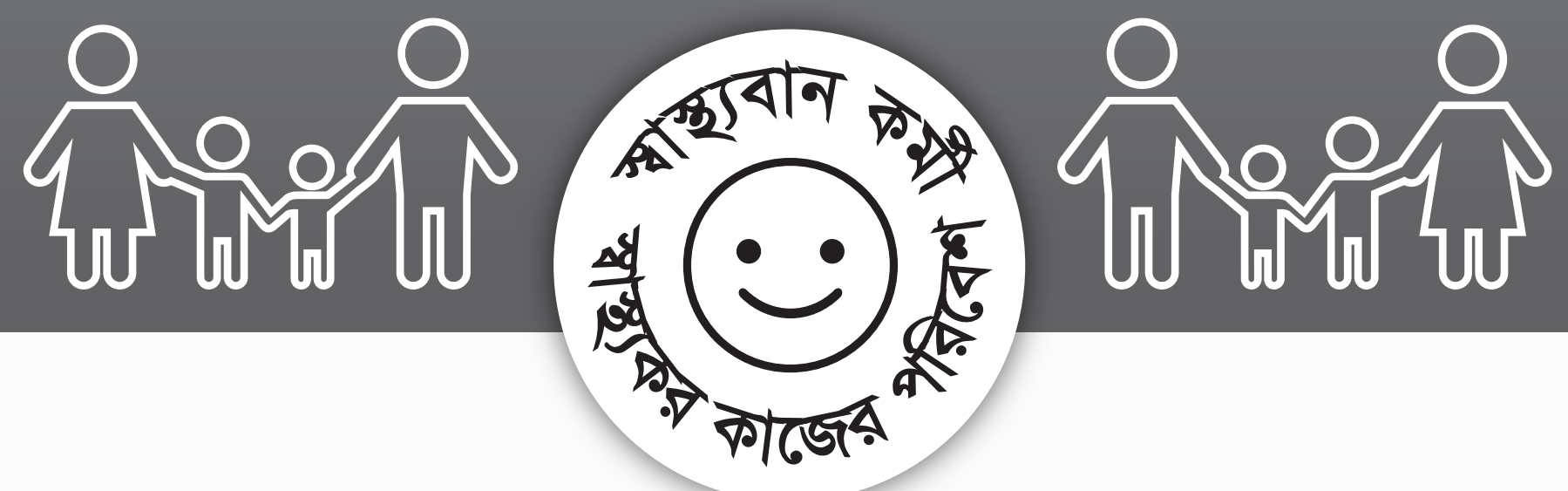

\section{পরিবার পরিকল্পনা কোন সুযোগ নয় পছন্দমত পদ্ধতি গ্রহণ করা !}

\section{$?$}

আপনার জন্য সবচয়ে কার্যকর উপায় কী হতে পারে? यদি আপনি ;

- পরিকল্পিত পরিবার গঠন করতে চান

- অনাকাজ্ষিত গর্ভধারণ প্রতিরোধ করতে চান

- অনিরাপদ গর্ভপাত এড়াতে চান

\section{!}

\section{কর্মজীবী নারীরা আধুনিক}

পরিবার পরিকল্পনা পদ্ধতি ব্যবহার করেন!

0000000000

খাবার বড়ি

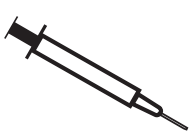

ইনজেকশন

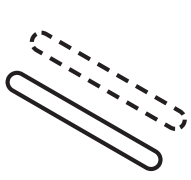

ইমপ্লান্ট

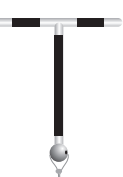

আইইউডি

আপনার কর্মন্য কিংবা বসবালর এলাকায় কর্মরত

স্বাম্ছ্য ও পরিবার কল্যাণ লবাদানকরীর সাথে কথা বলুন 

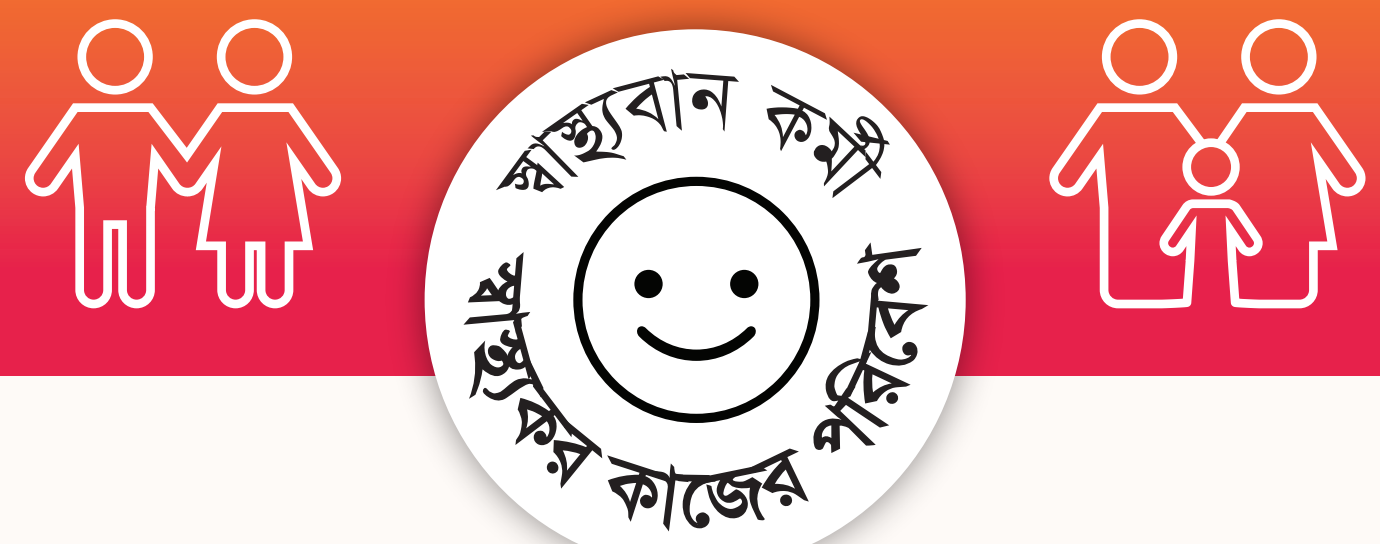

\section{আপনার সন্তানদের জন্য আরও সময় ও অর্থ নিশ্চিত করুন স্বাম্য্যবান পরিবার গড়ে হুলুন !}

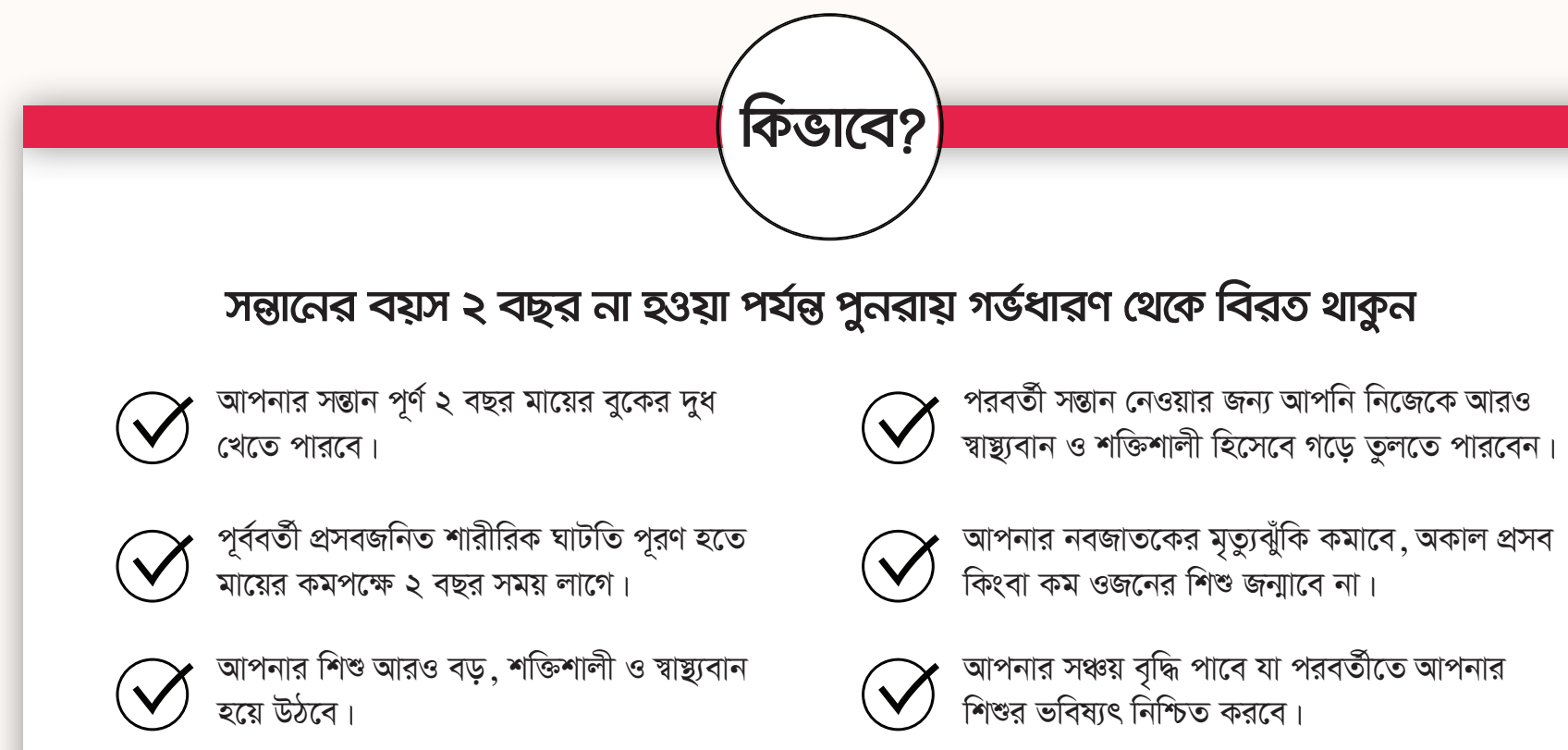

পরিবার পরিকল্পনার কোন পদ্ধতিটি আপনার জন্য সবळয়ে বেশি উপযোগী?

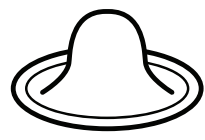

কনডম

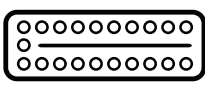

খাবার বড়ি

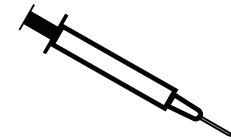

ইনজেকশন

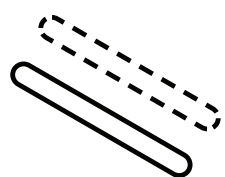

ইমপ্লান্ট

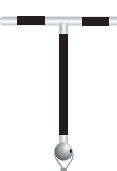

আইইউডি

আপনার কর্মন্ইল কিংবা বসবালর এলাকায় কর্মরত

স্বাম্য্য ও পরিবার কল্যাণ সেবাদানকরীর সাথ্য কথা বলুন

(48) 

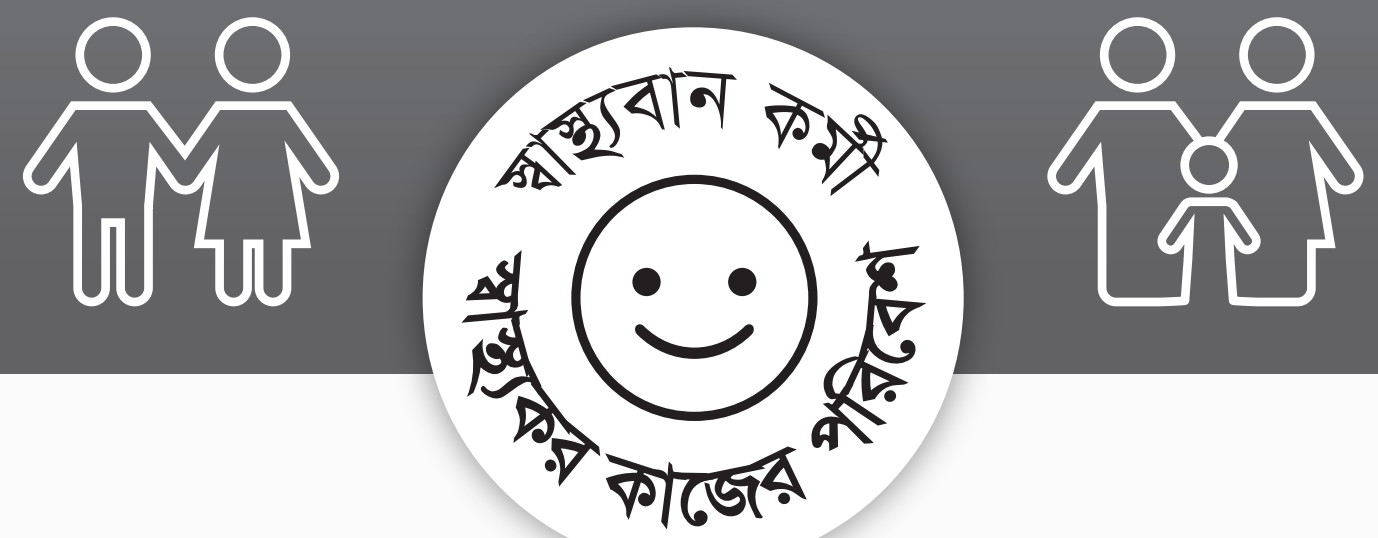

আপনার সন্তানদের জন্য আরও সময় ও অর্থ নিশ্চিত করুন স্বান্থ্যবান পরিবার গড়ে হুলুন !

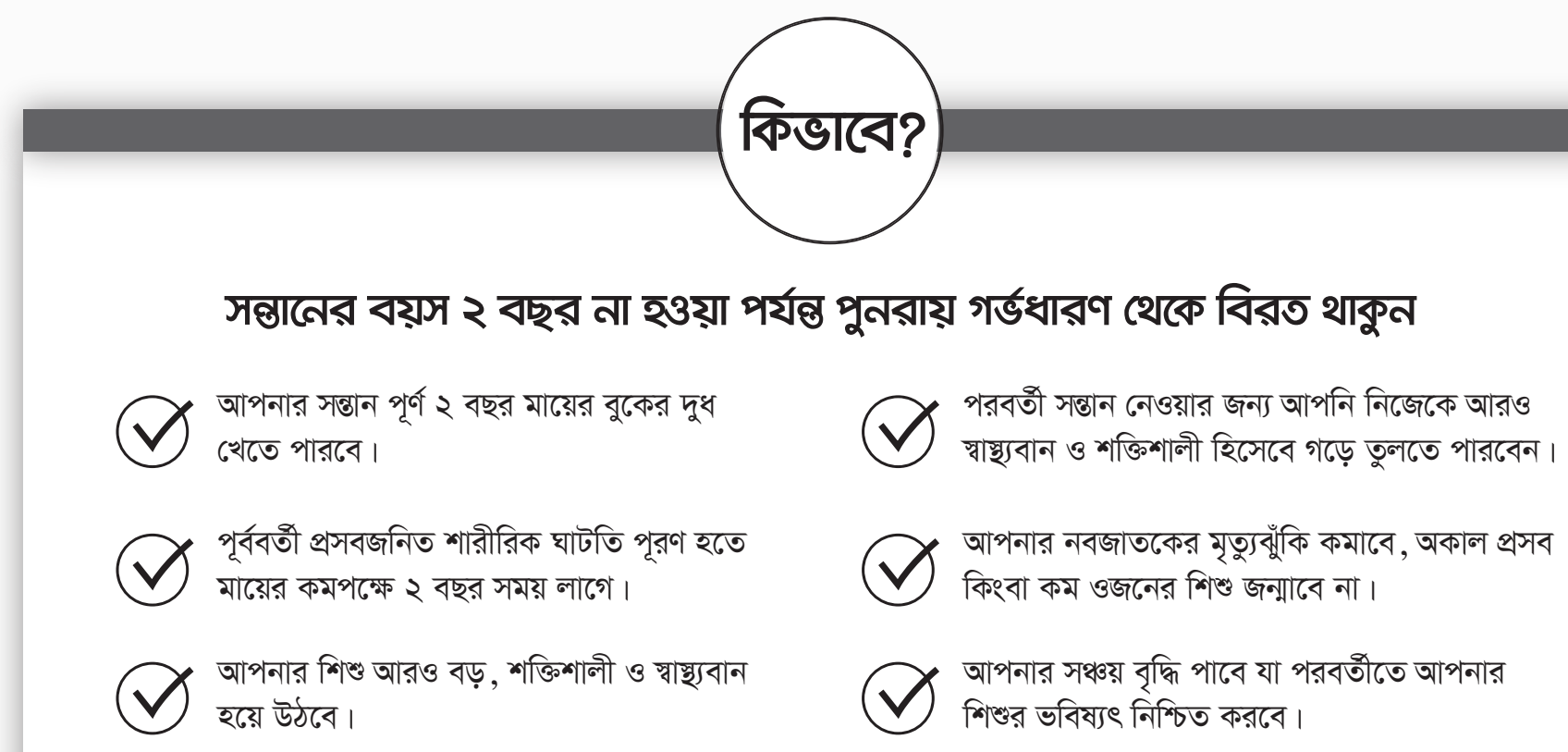

পরিবার পরিকল্পনার কোন পদ্ধতিটি আপনার জন্য সবळয়ে বেশি উপযোগী?

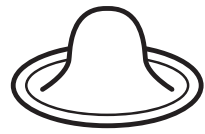

কনডম

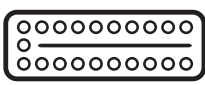

খাবার বড়ি

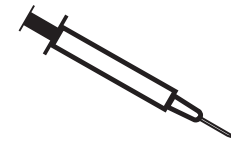

ইনজেকশন

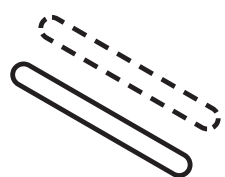

ইমপ্লান্ট

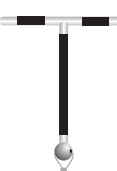

আইইউডি

আপনার কর্মন্ইল কিংবা বসবা৫র এলাকায় কর্মরত

স্বান্য্য ও পরিবার কল্যাণ সবাদানকরীর সাথ কথা বলুন 

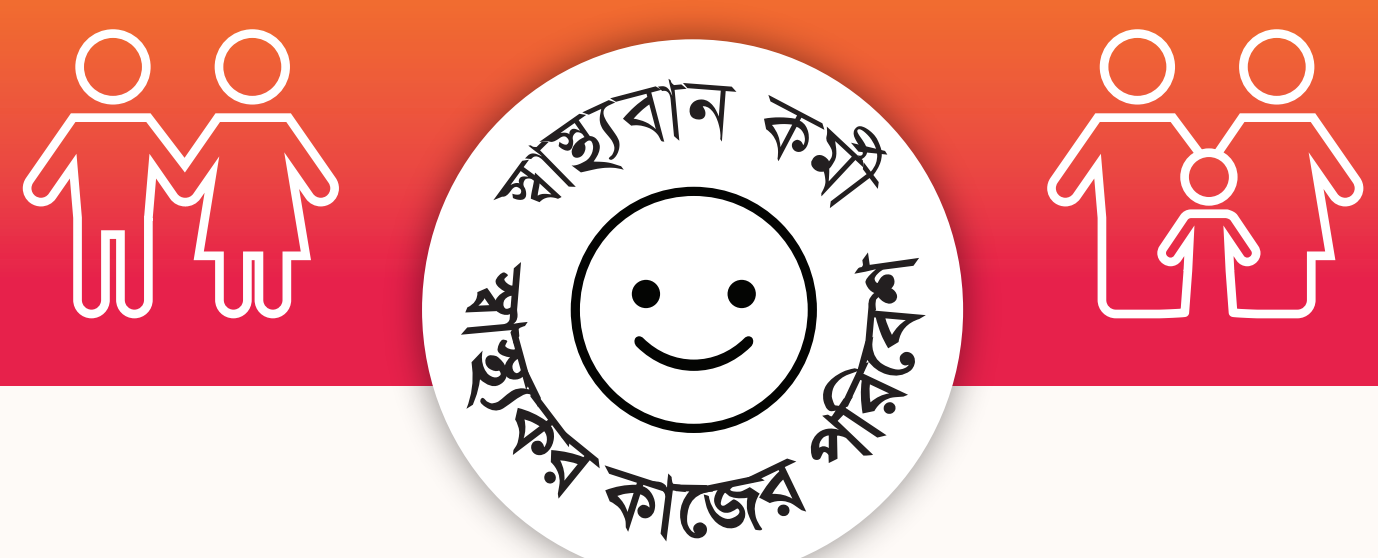

\section{স্বাস্থ্য সচ্নন কমীরা তাদের সন্তানদের বয়লের মধ্যে ব্যবধান রাখেন !}

\section{?}

কিভাবে একটি সুন্থ শিশু এবং

একটি সুন্থ পরিবার পাওয়া যায়?

\section{!}

- সন্তানের বয়স ২ বছর না হওয়া পর্যন্ত পুনরায় গর্ভধারণ থেকে বিরত থাকলে।

- আপনার পছন্দমত একটি আধুনিক পরিবার পরিকল্পনা পদ্ধতি ব্যবহার করলে।

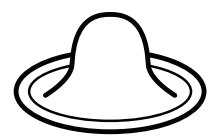

কনডম

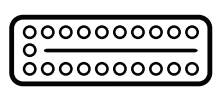

খাবার বড়ি

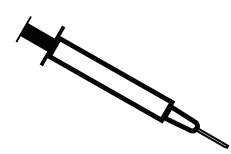

ইনজেকশন

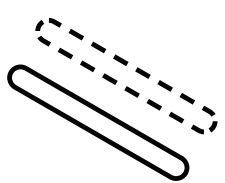

ইমপ্লান্ট

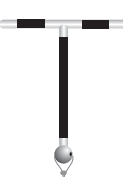

আইইউডি

আপনার কর্মছুল কিংবা বসবালর এলাকায় কর্মরত

ঘ্বান্য্য ও পরিবার কল্যাণ লেবাদানকারীর সাথ্য কথা বলুন

(40) 

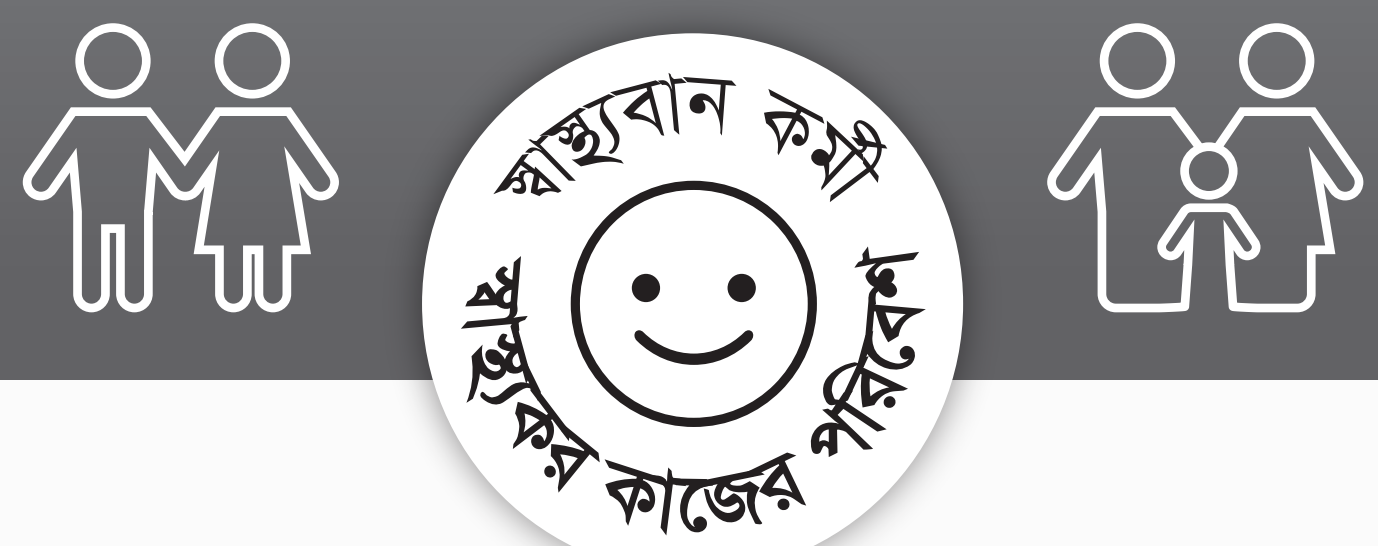

\section{স্বাস্থ্য সচ্নন কমীরা তাদের সন্তানদের বয়সের মধ্যে ব্যবধান রাখেন !}

\section{?}

কিভাবে একটি সুন্থ শিশু এবং

একটি সুন্থ পরিবার পাওয়া যায়?

\section{!}

- সন্তানের বয়স ২ বছর না হওয়া পর্যন্ত পুনরায় গর্ভধারণ থেকে বিরত থাকলে।

- আপনার পছন্দমত একটি আধুনিক পরিবার পরিকল্পনা পদ্ধতি ব্যবহার করলে।

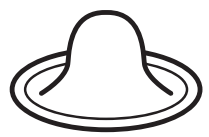

কনডম

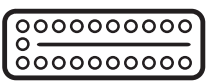

খাবার বড়ি

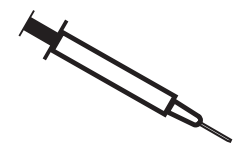

ইনজেকশন

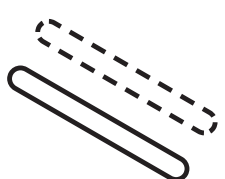

ইমপ্লান্ট

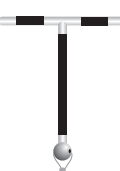

আইইউডি

আপনার কর্মছুল কিংবা বসবালর এলাকায় কর্মরত

ম্বান্ছ্য ও পরিবার কল্যাণ সেবাদানকারীর সাথে কথা বলুন 


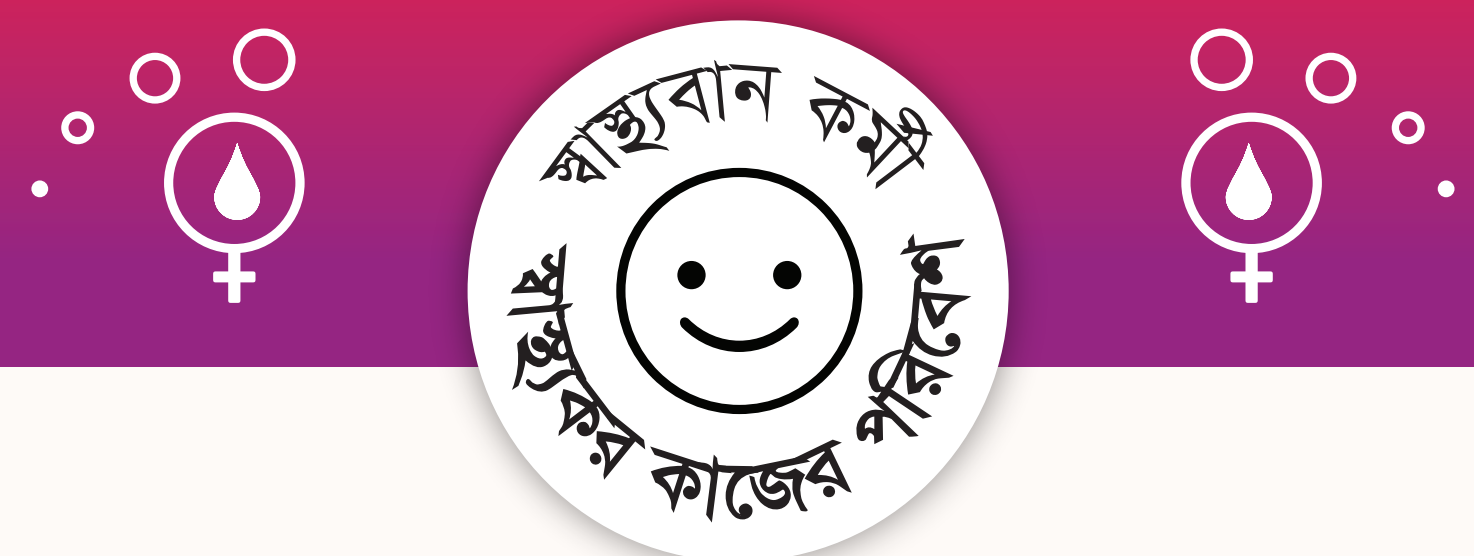

\section{মাসিক চলাকালে কি করতে হবে আপনি জানেন কি? নিজেকে যাচাই করুন:}

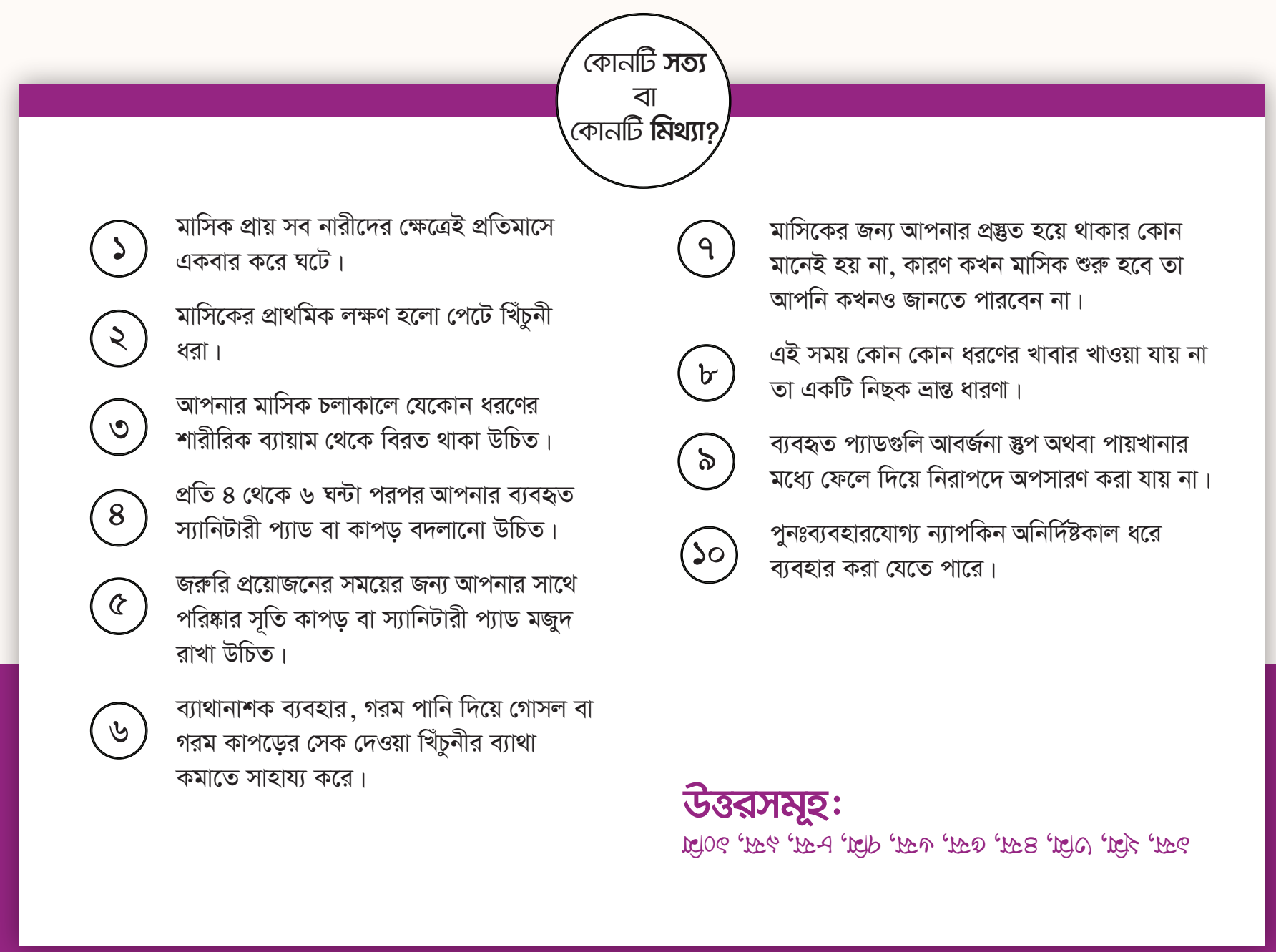

\footnotetext{
আপনার কর্মন্নল কিংবা বসবালর এলাকায় কর্মরত ম্বান্য্য ও পরিবার কল্যাণ লসবাদানকারীর সাথে কথা বলুন
} 


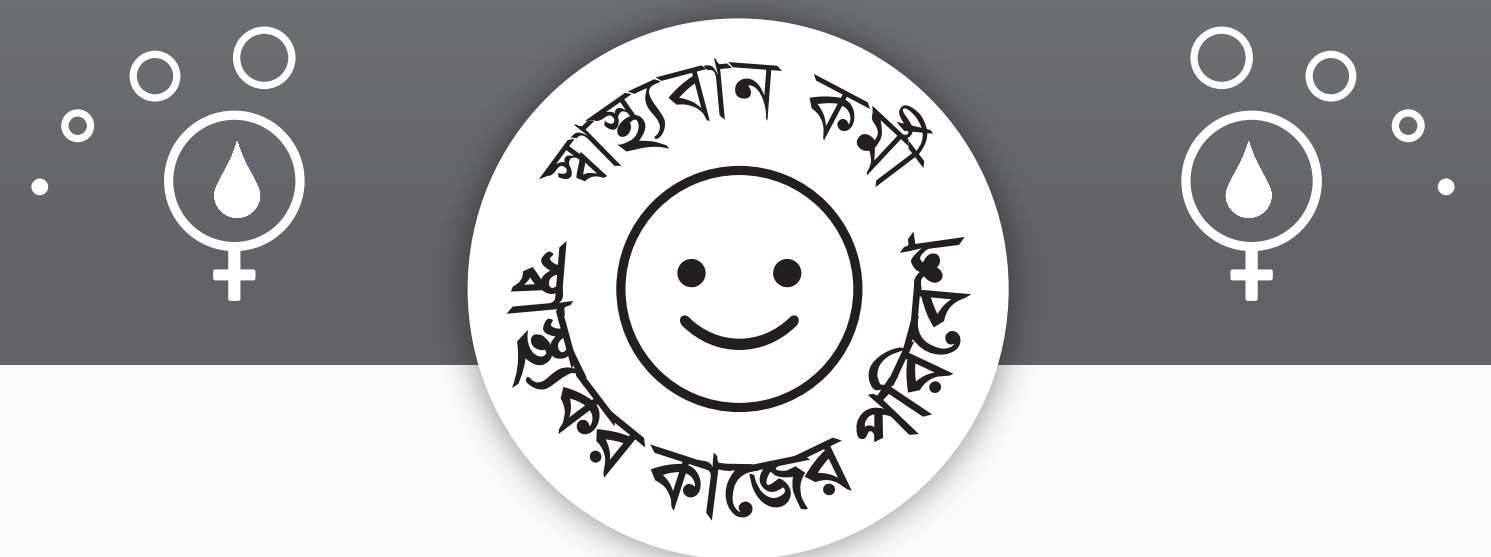

\section{মাসিক চলাকালে কি করতে হবে আপনি জানেন কি? নিজেকে যাচাই করুন:}

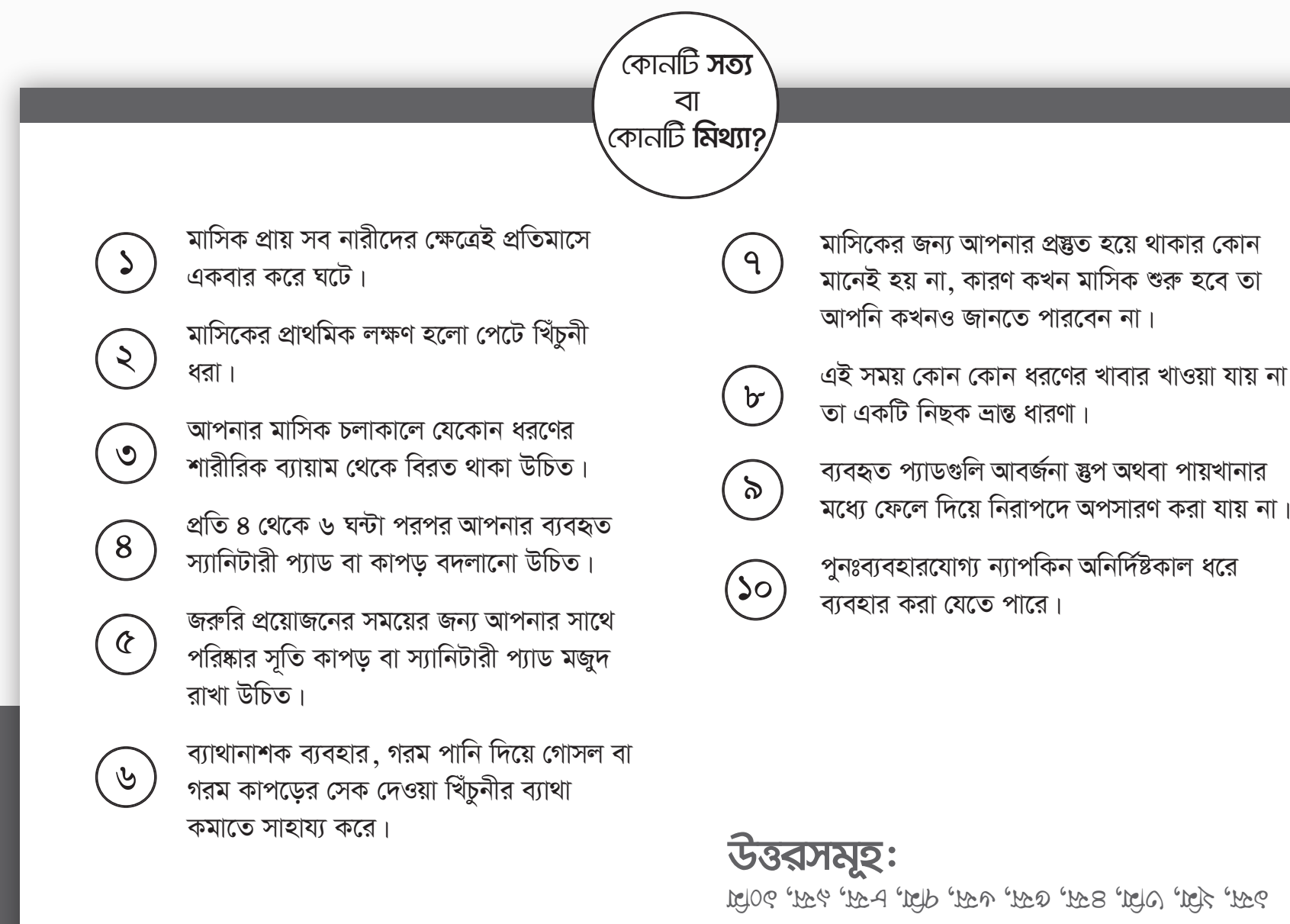

\footnotetext{
আপনার কর্মন্ইল কিংবা বসবাসর এলাকায় কর্মরত স্বান্য্য ও পরিবার কল্যাণ সবাদানকরীর সাথে কথা বলুন
} 


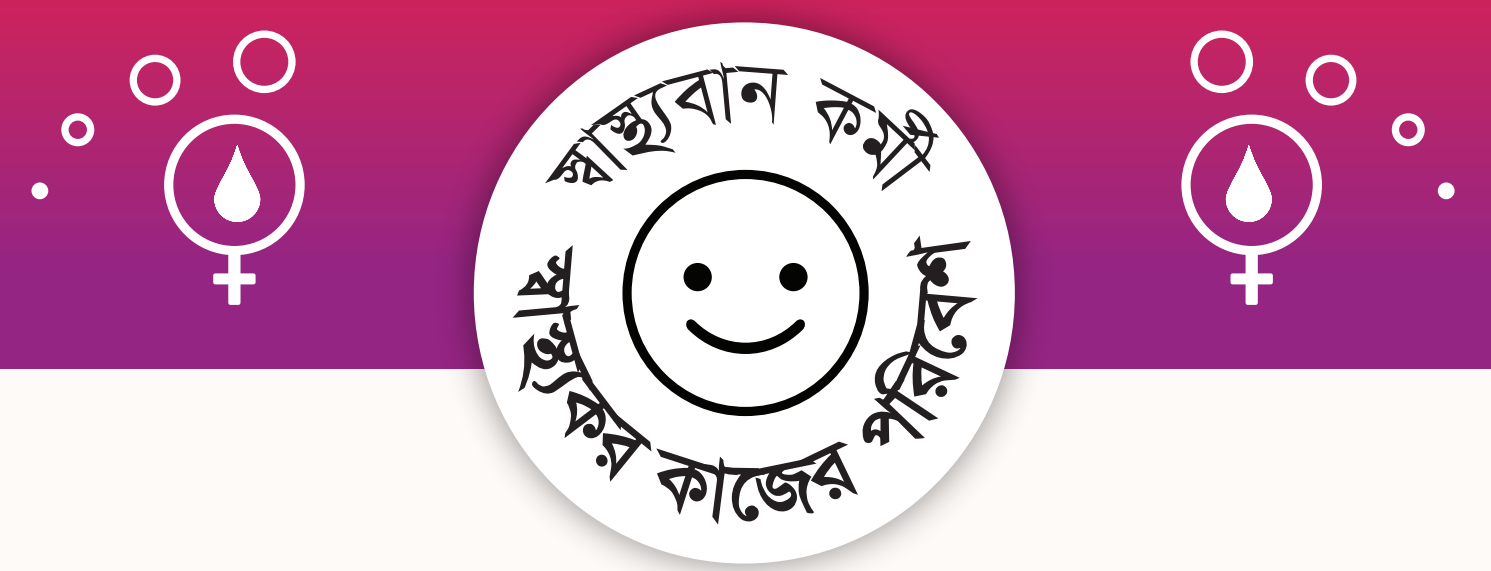

\section{মাসিক চলাকাল সুম্থ থাকুন}

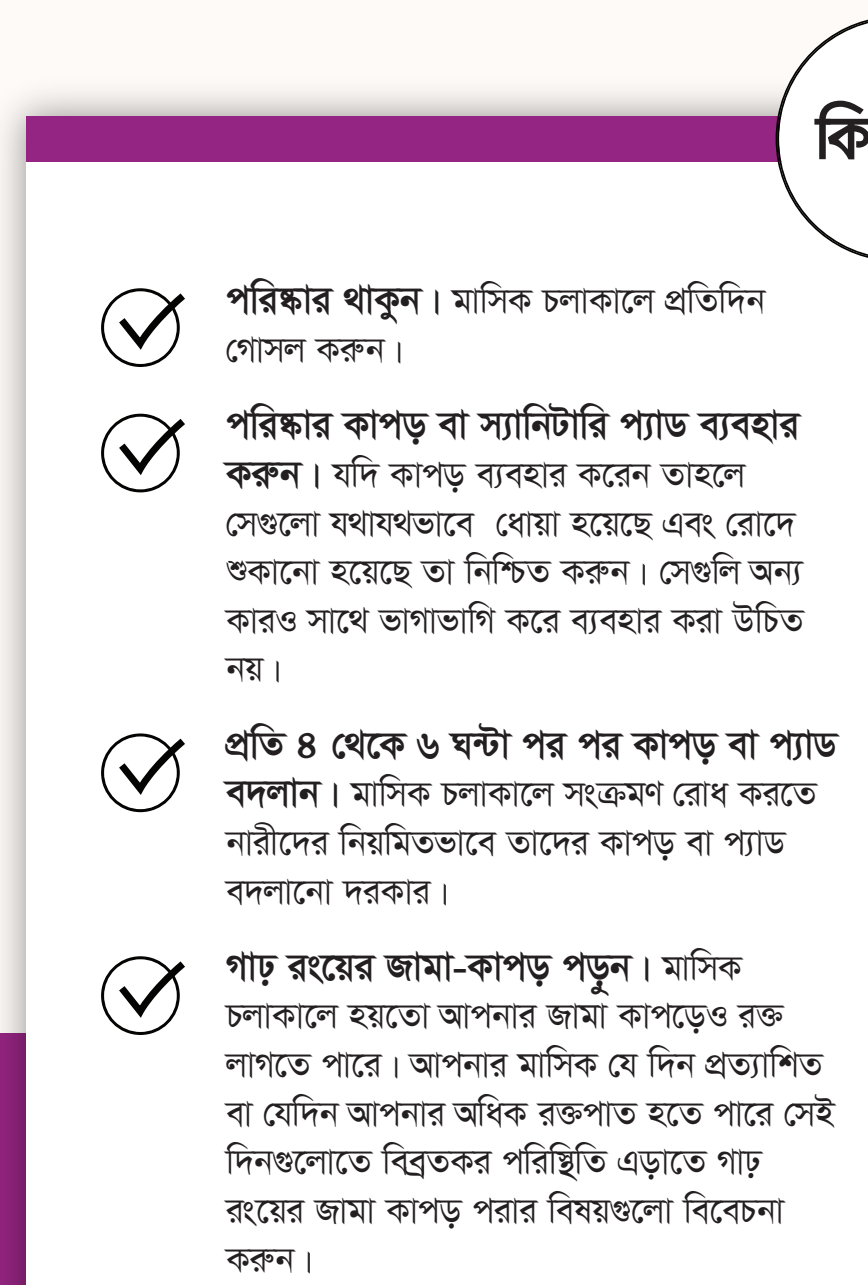

কষ কমাতে ব্যাথানাশক ব্যবহার করুন, গরম পানি দিয়ে গোসল করুন অথবা হালকা সেক দিয়ে মালিশ করুন। কারও কারও ক্ষেত্রে তলপেটে ব্যাথায় খিল ধরতে পারে।

ব্যায়াম করা ও হালকা মালিশ করা, দুটি কার্যক্রমই তলপেটে খিল ধরার উপশমের ক্ষেত্রে আরাম প্রদান করতে পারে।

প্রস্তুত থাকুন। আপনার কাজের জায়গাতেও মাসিক শুরু হতে পারে। আপনি যদি আপদকালীন সময়ে প্যাড বা বিকল্প কিছু এবং ব্যাথানাশক সাথে রাখেন, তাহলে নিয়মিত কাজ চালিয়ে যেতে পারবেন। সঠিকভাবে খাওয়া- দাওয়া করুন। এই সময় কোন কোন ধরণের খাবার খাওয়া যায় না বলে যা বলা হয় তা নিছক একটি ভ্রান্ত ধারণা। রক্তক্ষরণের ক্ষতিপূরণ করতে ও রক্তস্বল্পতা রোধ করতে আয়রণ সমৃদ্ধ খাবার খাওয়া প্রয়োজন। ফলমূল বা শাকসবিজ বেশি বেশি করে খাওয়া উচিত। বেশিবেশি চা কফি এবং লবণ খাওয়া থেকে বিরত থাকুন। করুন্ন।

\section{আপনার কর্মন্ইল কিংবা বসবালর এলাকায় কর্মরত স্বাষ্ছ্য ও পরিবার কল্যাণ সবাদানকারীর সাথ কথা বলুন}




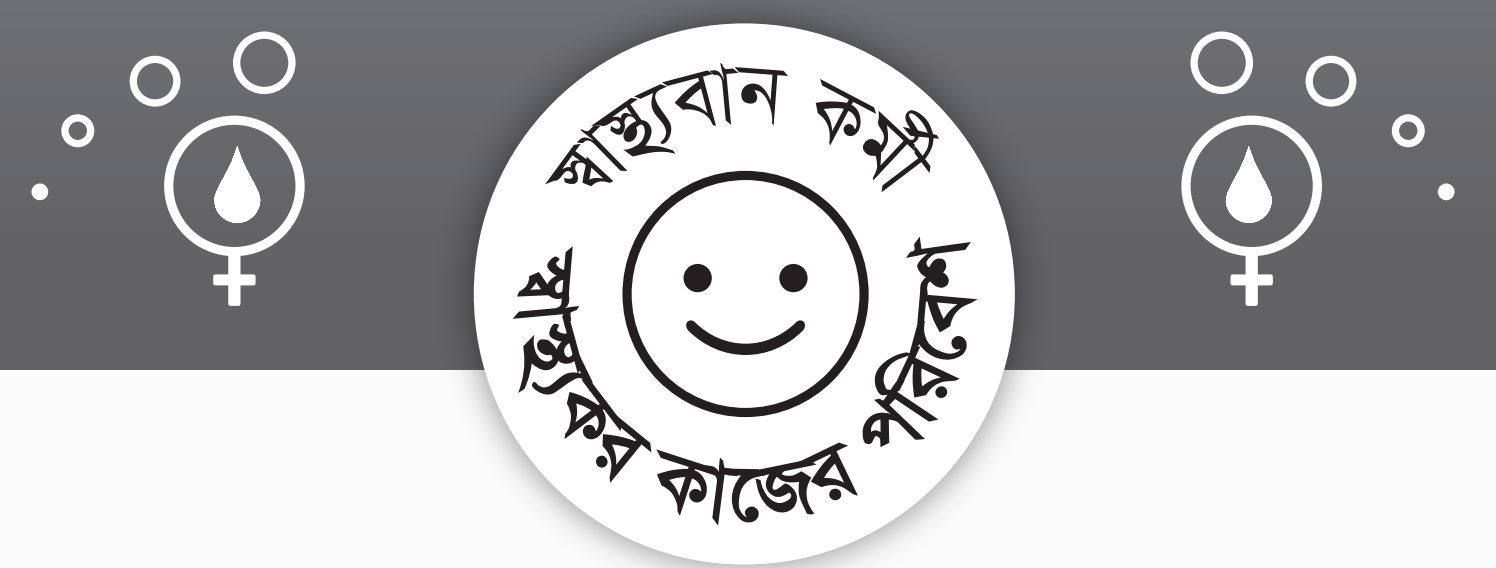

\section{মাসিক চলাকাল সুম্থ থাকুন}

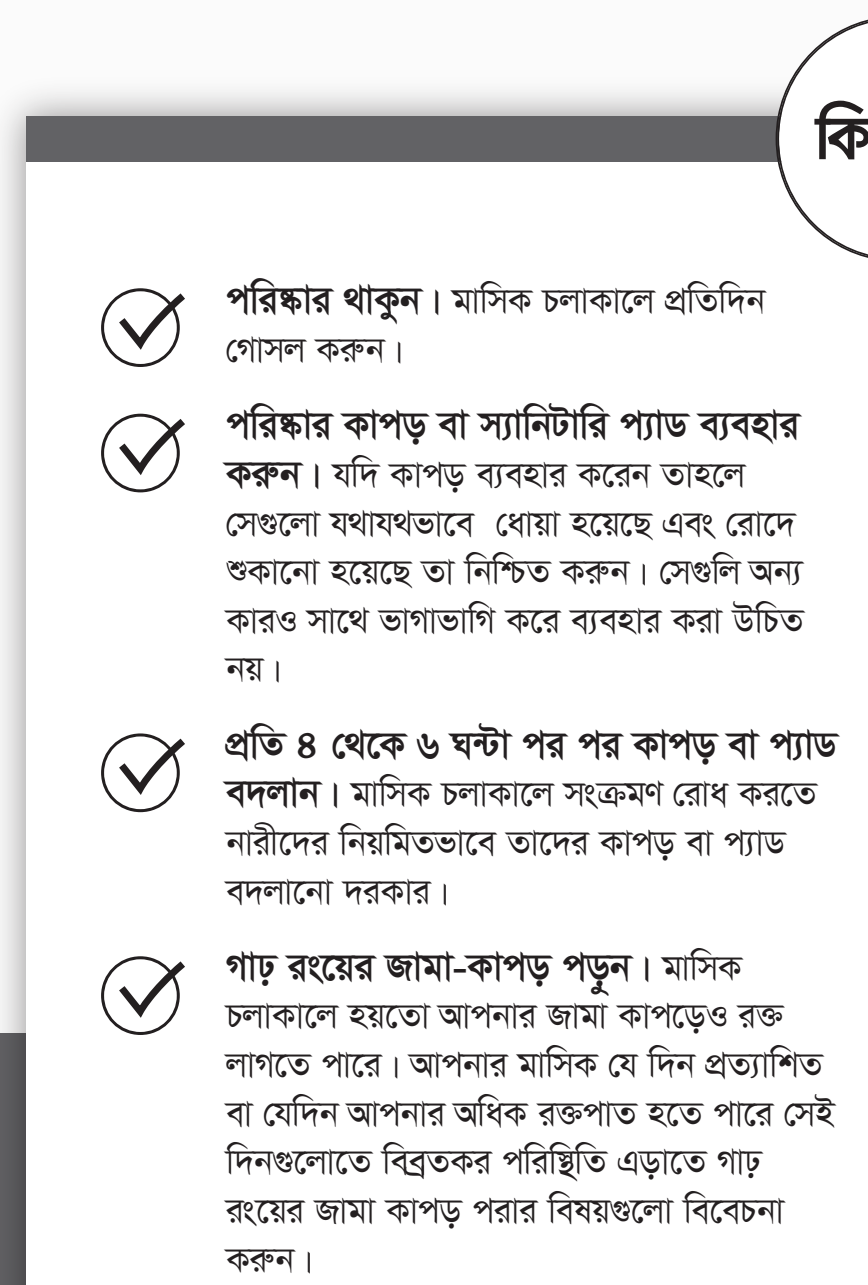

কষ কমাতে ব্যাথানাশক ব্যবহার করুন, গরম পানি দিয়ে গোসল করুন অথবা হালকা সেক দিয়ে মালিশ করুন। কারও কারও ক্ষেত্রে তলপেটে ব্যাথায় খিল ধরতে পারে।

ব্যায়াম করা ও হালকা মালিশ করা, দুটি কার্যক্রমই তলপেটে খিল ধরার উপশমের ক্ষেত্রে আরাম প্রদান করতে পারে।

প্রস্তুত থাকুন। আপনার কাজের জায়গাতেও মাসিক শুরু হতে পারে। আপনি যদি আপদকালীন সময়ে প্যাড বা বিকল্প কিছু এবং ব্যাথানাশক সাথে রাখেন, তাহলে নিয়মিত কাজ চালিয়ে যেতে পারবেন। সঠিকভাবে খাওয়া- দাওয়া করুন্ন। এই সময় কোন কোন ধরণের খাবার খাওয়া যায় না বলে যা বলা হয় তা নিছক একটি ভ্রান্ত ধারণা। রক্তক্ষরণের ক্ষতিপূরণ করতে ও রক্তস্বল্পতা রোধ করতে আয়রণ সমৃদ্ধ খাবার খাওয়া প্রয়োজন। ফলমূল বা শাকসবিজ বেশি বেশি করে খাওয়া উচিত। বেশিবেশি চা কফি এবং লবণ খাওয়া থেকে বিরত থাকুন। করুন্ন।

\section{আপনার কর্মন্ইল কিংবা বসবালর এলাকায় কর্মরত স্বাষ্ছ্য ও পরিবার কল্যাণ সবাদানকারীর সাথ কথা বলুন}




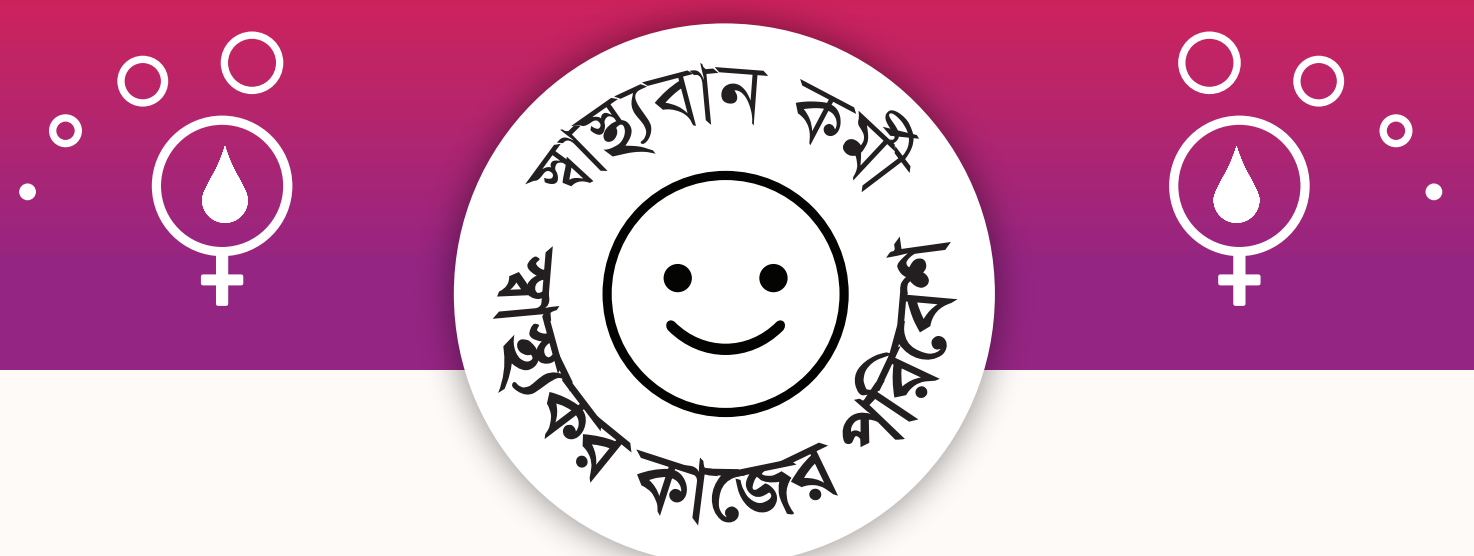

\section{নিজেকে সুস্থ রাখুন আপনার মাসিক চলাকালে!}

\section{?}

মাসিক চলাকাল নারী কমীরা

কিভাবে নিজেদেরকে সুস্থ রাখতে পারেন?

\section{!}

আপনার মাসিক চলাকালে কি করতে হবে এবং

কিভাবে ভালো থাকতে পারেন তা জেনে নিন :

- পরিষ্কার কাপড় বা স্যানিটারি প্যাড ব্যবহার করুন

- প্রতি 8 থেকে ৬ ঘন্টা পরপর কাপড় বা প্যাড বদলান

- কষ্ট কমাতে গরম পানি দিয়ে গোসল করুন অথবা হালকা গরম কাপড়ের সেক দিয়ে মালিশ করুন অথবা ব্যাথানাশক ওযুধ খান

- ব্যবহৃত প্যাড বা কাপড় সঠিকভাবে অপসারণ করুন

- প্রতিদিনের স্বাভাবিক পুষ্টিকর খাবার খান

আপনার কর্মইল কিংবা বসবালর এলাকায় কর্মরত

স্বান্য্য ও পরিবার কল্যাণ লসবাদানকারীর সাথ কথা বলুন 


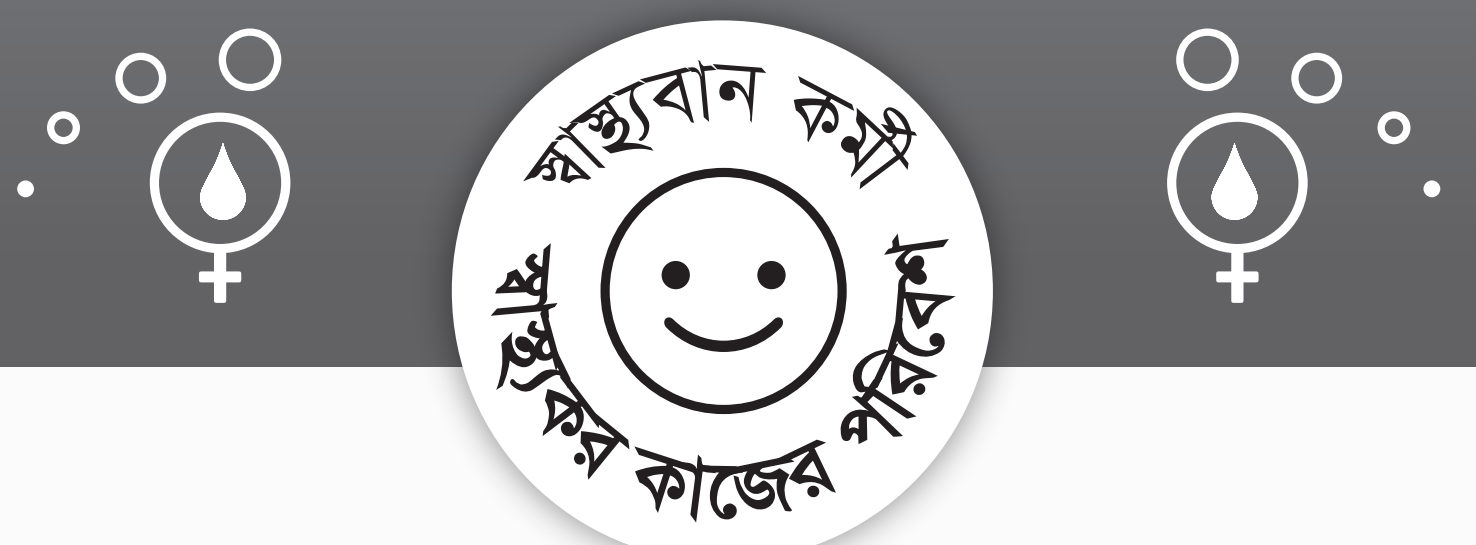

\section{নিজেকে সুস্থ রাখুন আপনার মাসিক চলাকালে!}

\section{?}

মাসিক চলাকাল নারী কমীরা

কিভাবে নিজেদেরকে সুস্থ রাখতে পারেন?

\section{!}

আপনার মাসিক চলাকালে কি করতে হবে এবং

কিভাবে ভালো থাকতে পারেন তা জেনে নিন:

- পরিষ্কার কাপড় বা স্যানিটারি প্যাড ব্যবহার করুন

- প্রতি 8 থেকে ৬ ঘন্টা পরপর কাপড় বা প্যাড বদলান

- কষ্ট কমাতে গরম পানি দিয়ে গোসল করুন অথবা হালকা গরম কাপড়ের সেক দিয়ে মালিশ করুন অথবা ব্যাথানাশক ওযুধ খান

- ব্যবহৃত প্যাড বা কাপড় সঠিকভাবে অপসারণ করুন

- প্রতিদিনের স্বাভাবিক পুষ্টিকর খাবার খান

আপনার কর্মইল কিংবা বসবালর এলাকায় কর্মরত

ম্বান্য্য ও পরিবার কল্যাণ লসবাদানকারীর সাথে কথা বলুন

(0.) 

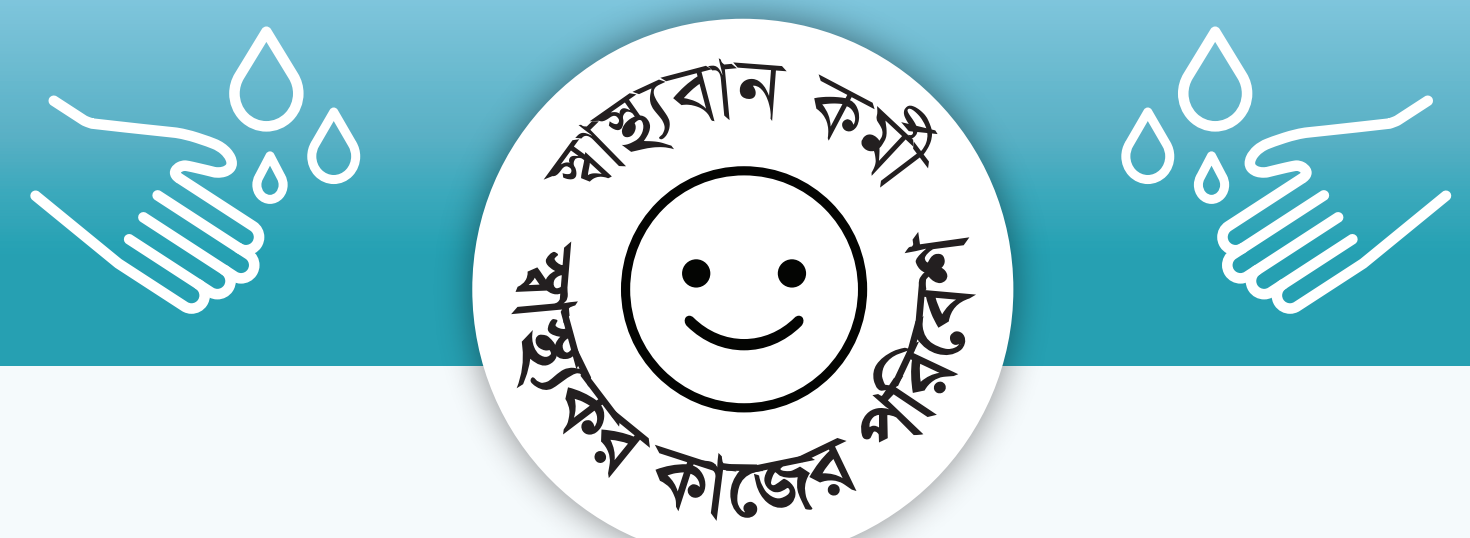

\section{আপনার দুই হাত ধুয়ে নিন !}

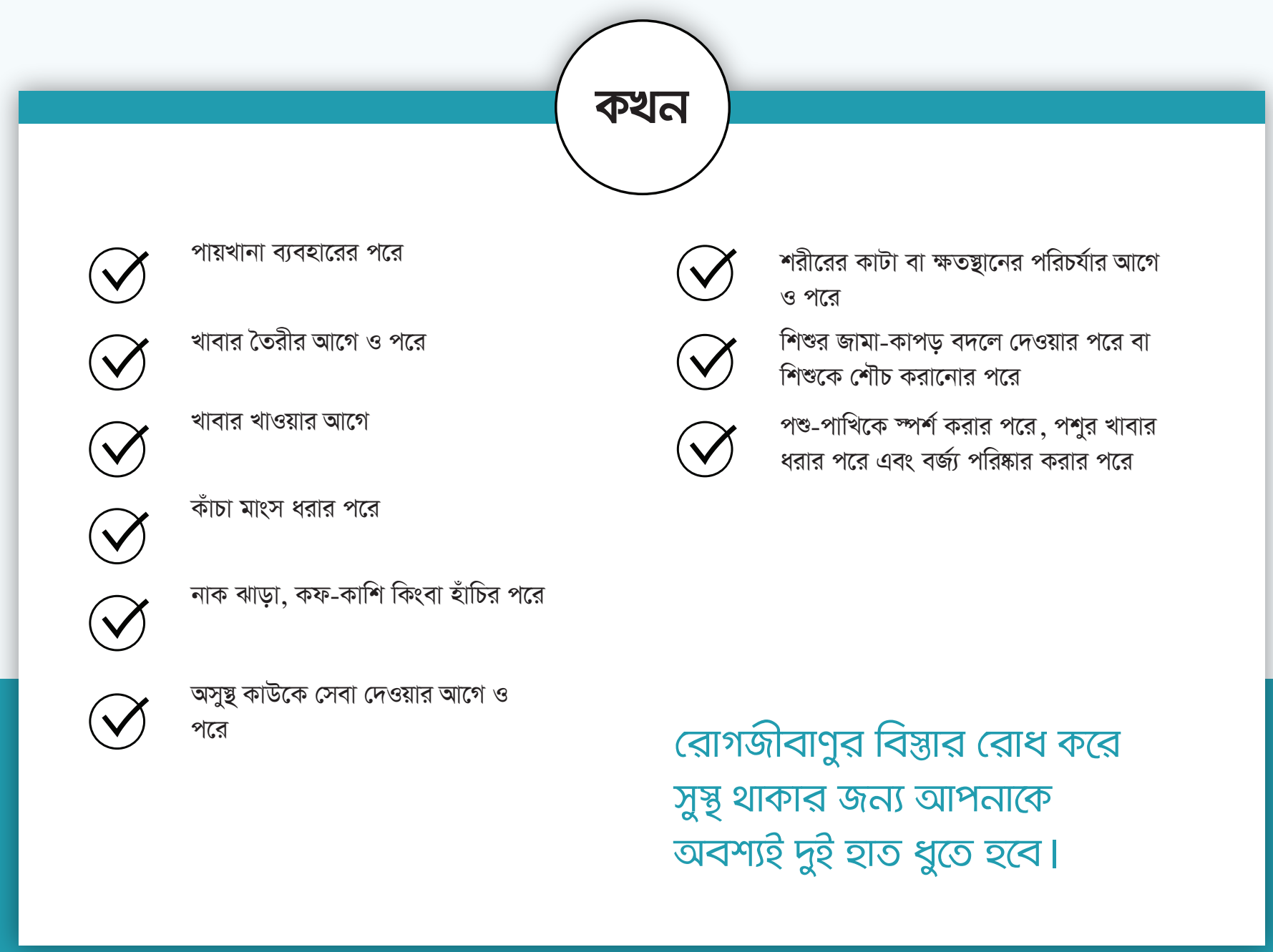

\section{আপনার কর্মন্ইল কিংবা বসবালর এলাকায় কর্মরত \\ স্বাষ্্য ও পরিবার কল্যাণ সববাদানকরীর সাথ কথা বলুন}

(3) 


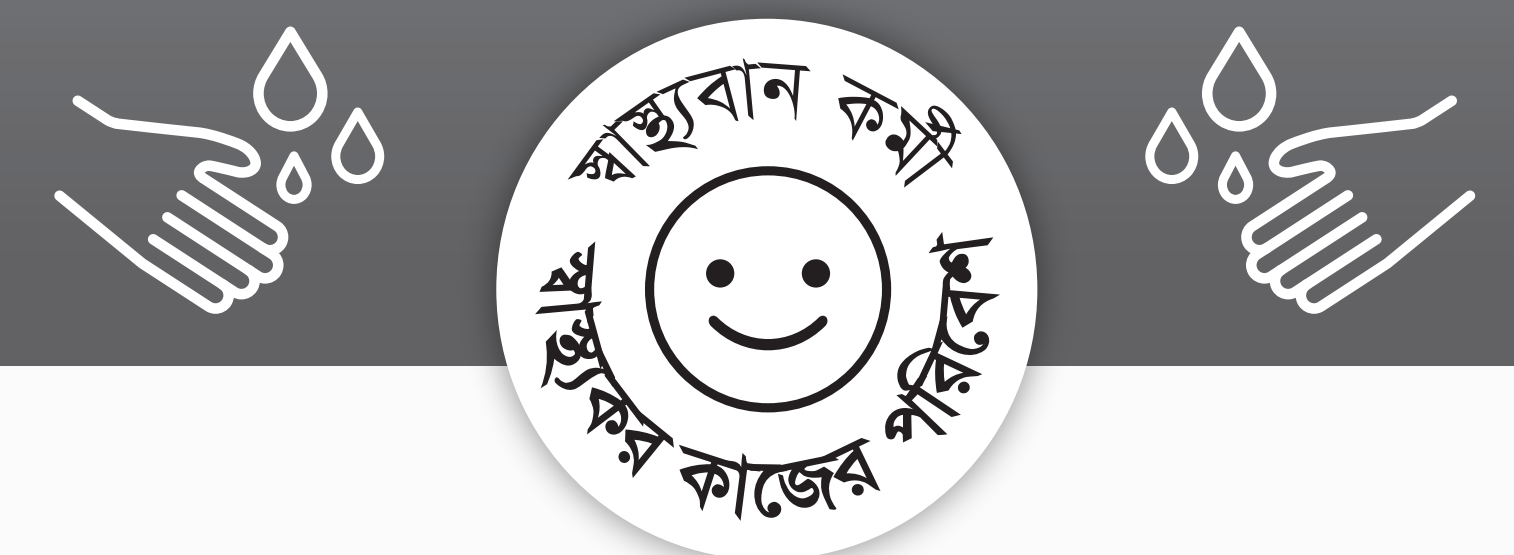

\section{আপনার দুই হাত ধুয়ে নিন !}

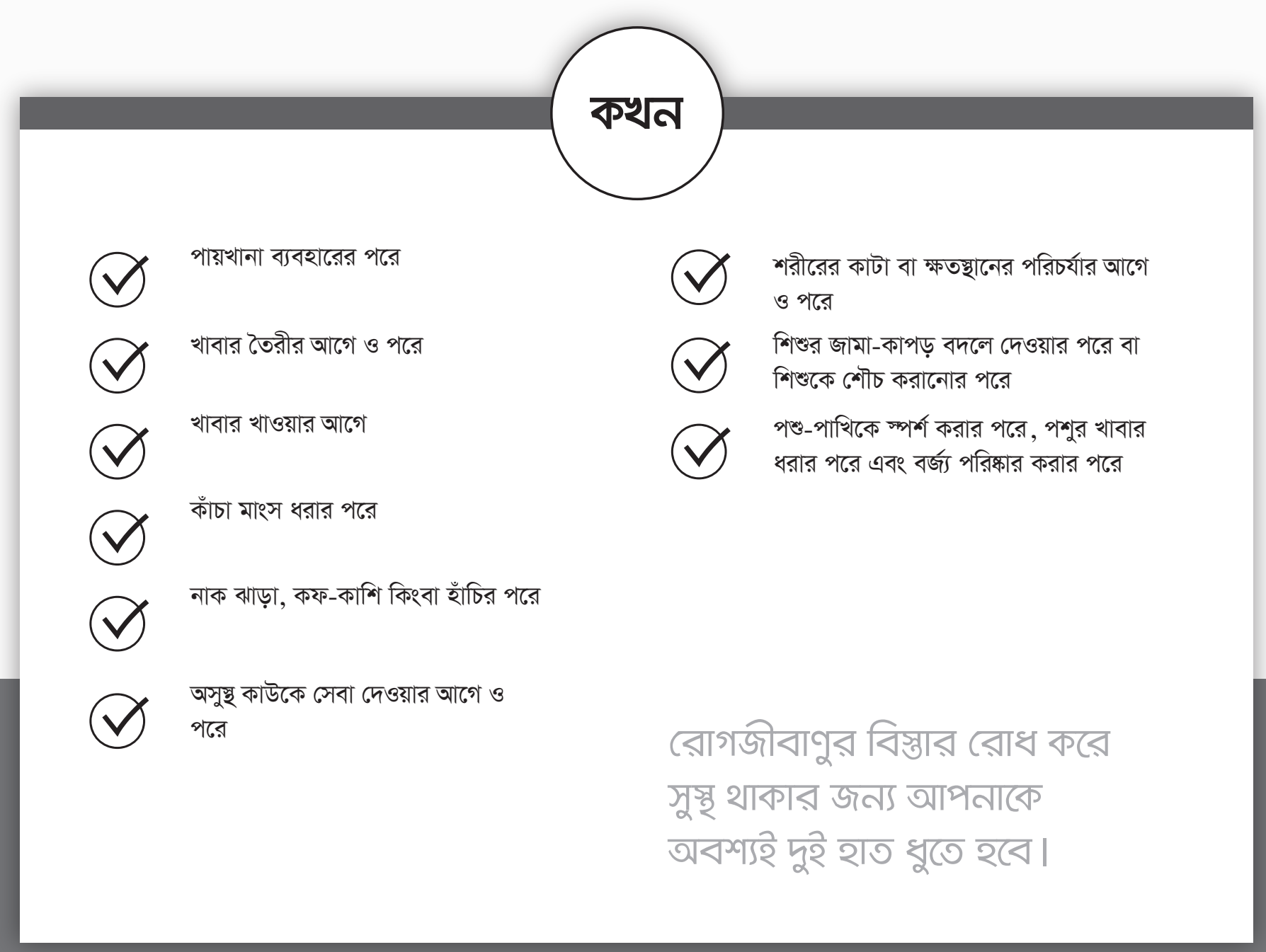

\footnotetext{
আপনার কর্মন্ইল কিংবা বসবালরর এলাকায় কর্মরত

ন্যা্্য ও পরিবার কল্যাণ লববাদানকারীর সাথে কথা বলুন
}

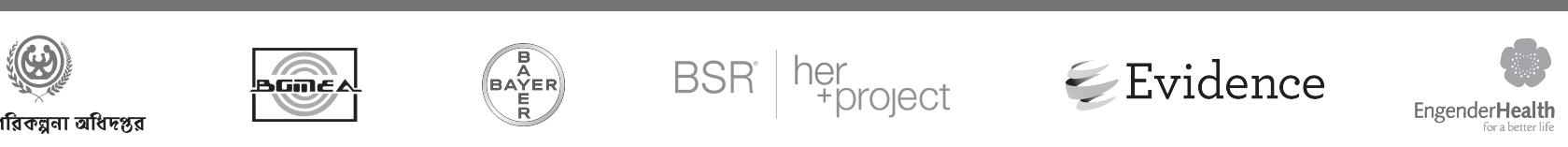



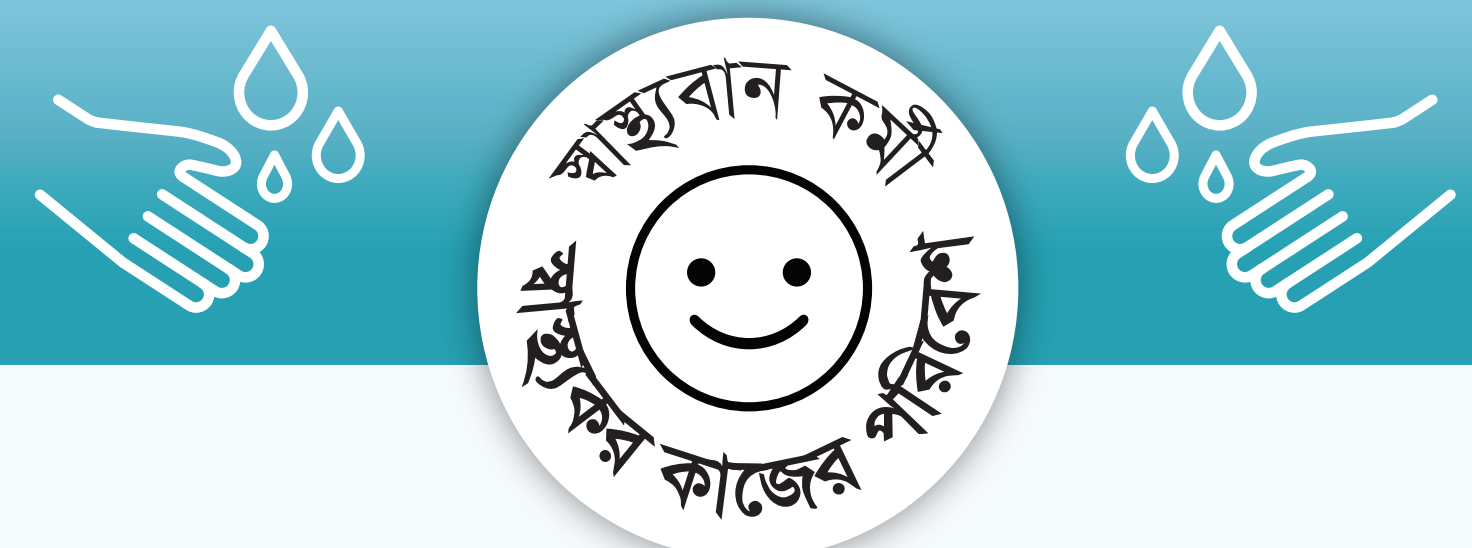

\section{আপনার দুই হাত ধুয়ে নিন !}

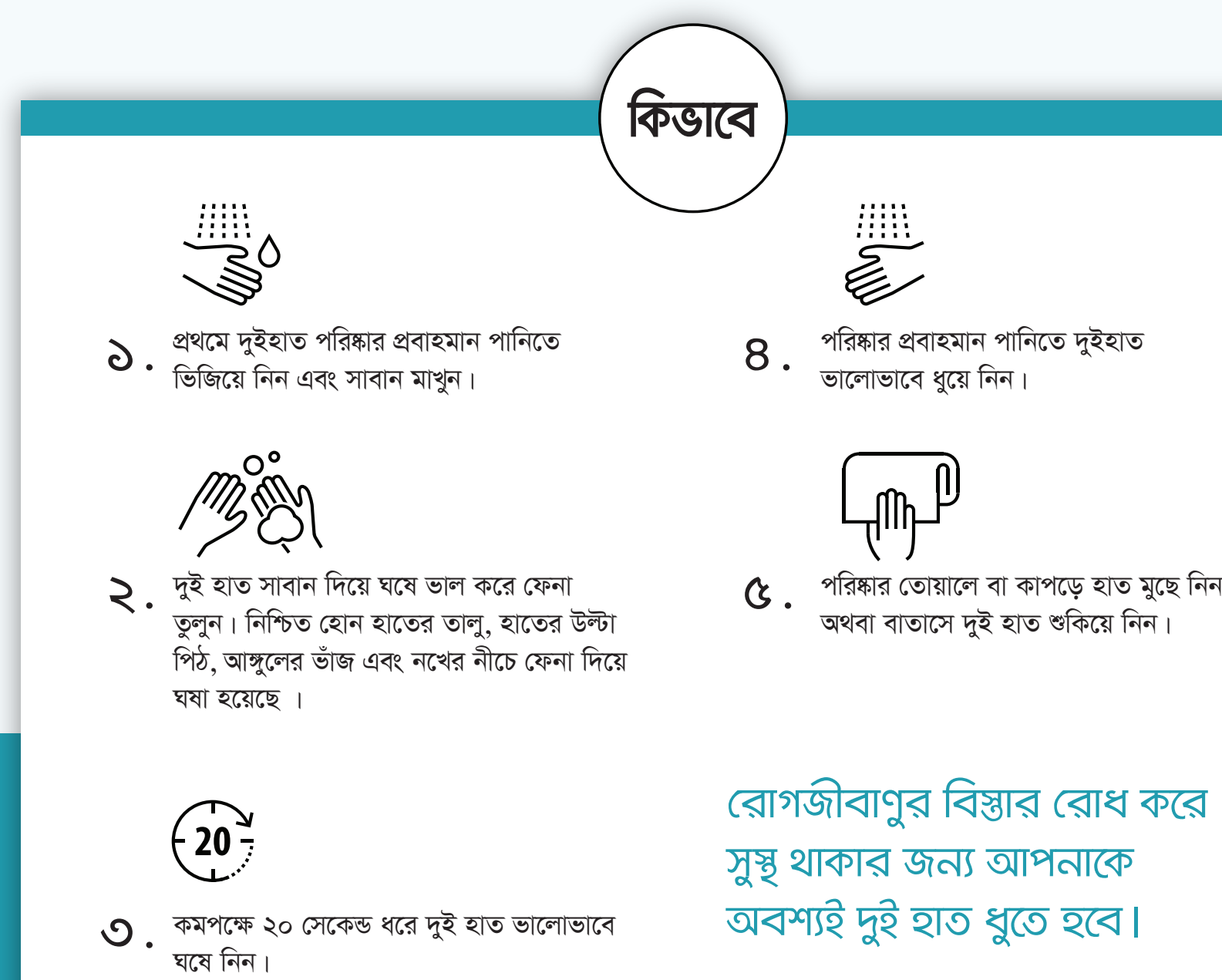

\section{আপনার কর্মন্ইল কিংবা বসবালর এলাকায় কর্মরত \\ স্বাষ্ছ্য ও পরিবার কল্যাণ লবাদানকরীর সাথে কথা বলুন}

(88) 


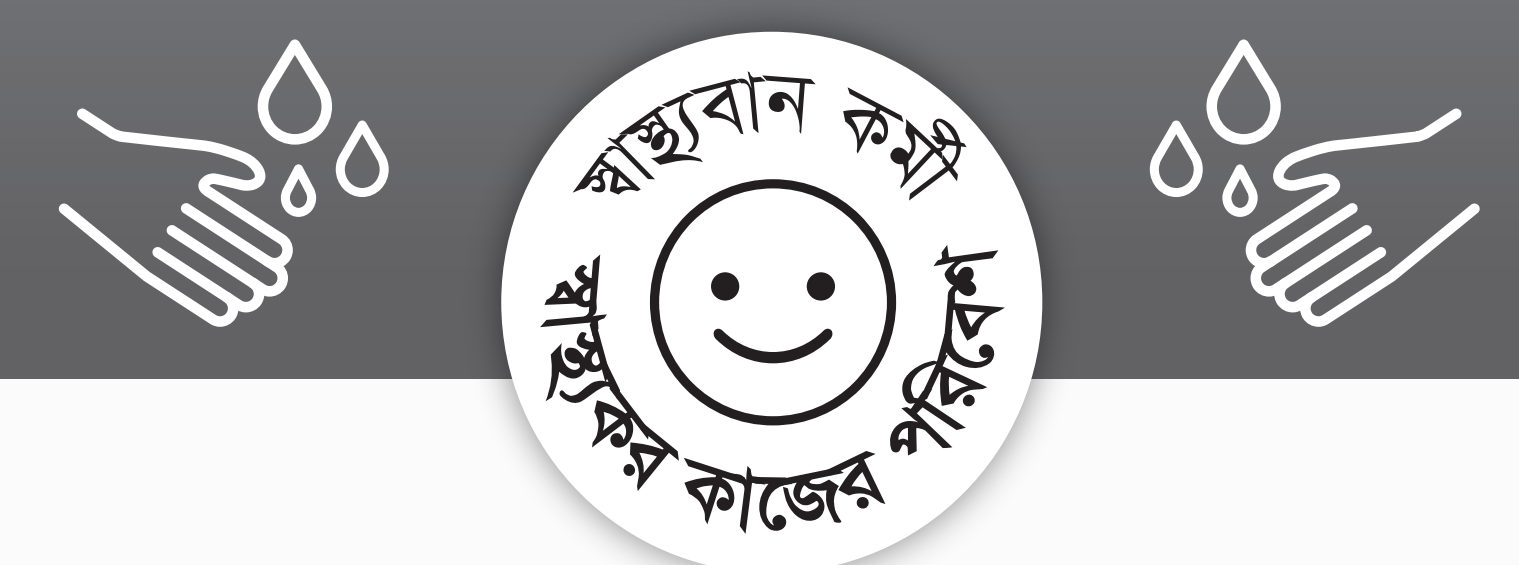

\section{আপনার দুই হাত ধুয়ে নিন !}

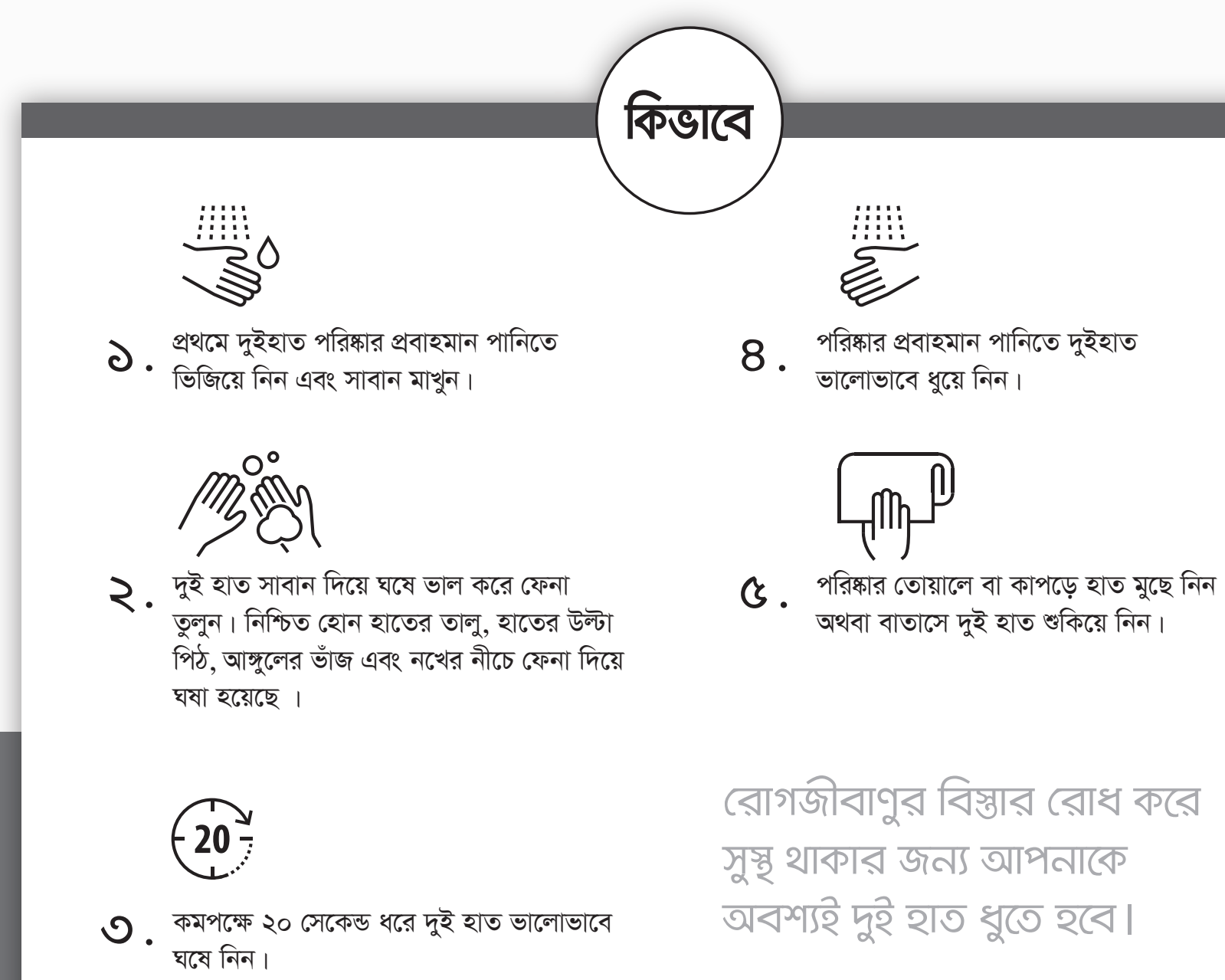

\footnotetext{
আপনার কর্মন্ইল কিংবা বসবালর এলাকায় কর্মরত

স্বাম্ছ্য ও পরিবার কল্যাণ সবাদানকারীর সাথে কথা বলুন
} 

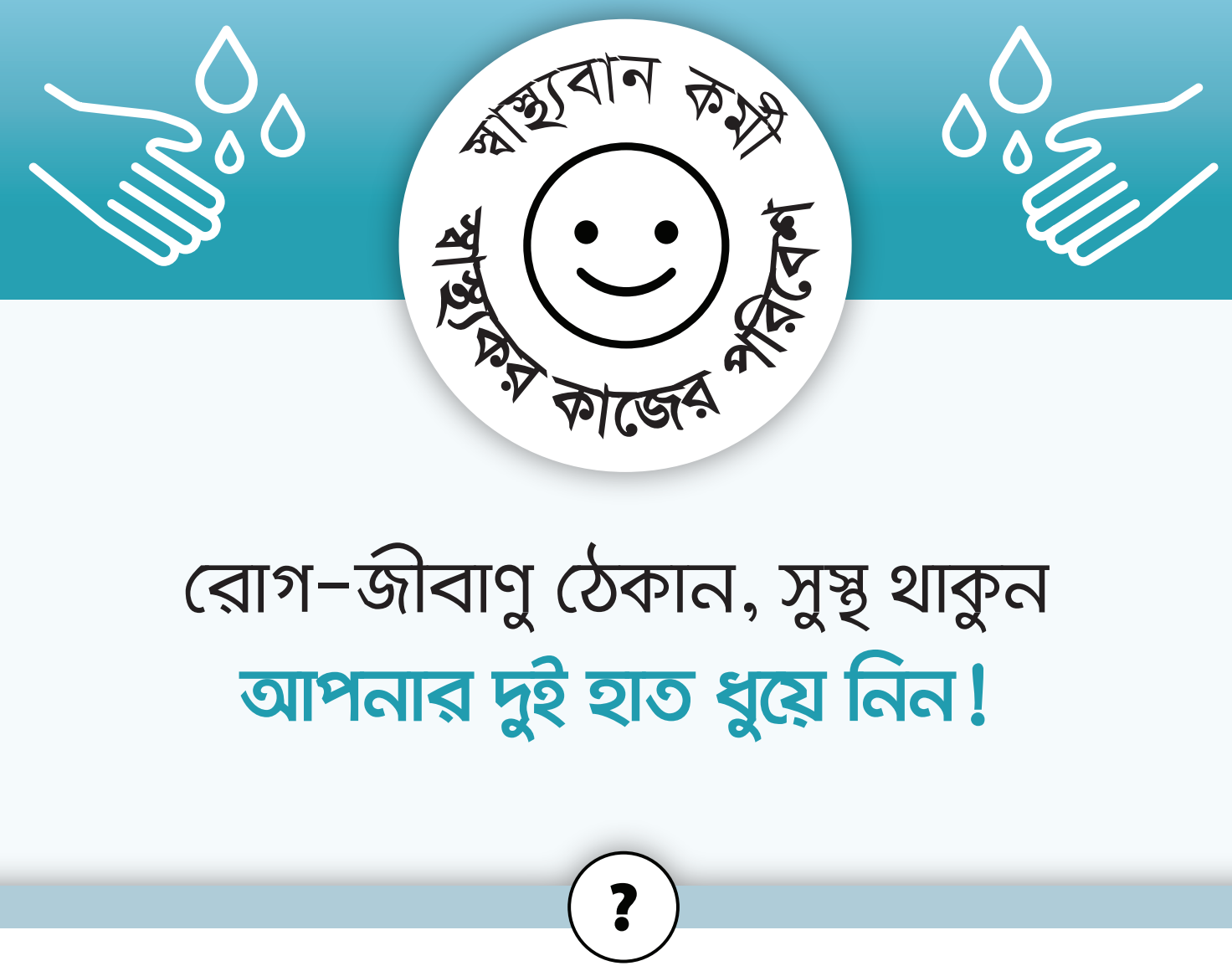

কখন আপনার দুই হাত ধোয়া উচিত?

\section{!}

আপনার দুই হাত ধোয়ার

\section{গুরুত্বপূর্ণ সময়গুলো হচ্ছে;}

- পায়খানা ব্যবহারের পরে

- খাবার তৈরীর আগে ও পরে

- খাবার খাওয়ার আগে

- কঁঁচা মাংস ধরার পরে

- নাক ঝাড়া, কফ-কাশি বা হাঁচির পরে

- পেশাগত সরঞ্জাম ও যন্ত্রপাতি ব্যবহারের পরে

\section{আপনার কর্মহুল কিংবা বসবা৫র এলাকায় কর্মরত \\ স্বাম্ছ্য ও পরিবার কল্যাণ সেবাদানকরীর সাথ কথা বলুন}

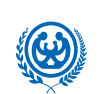



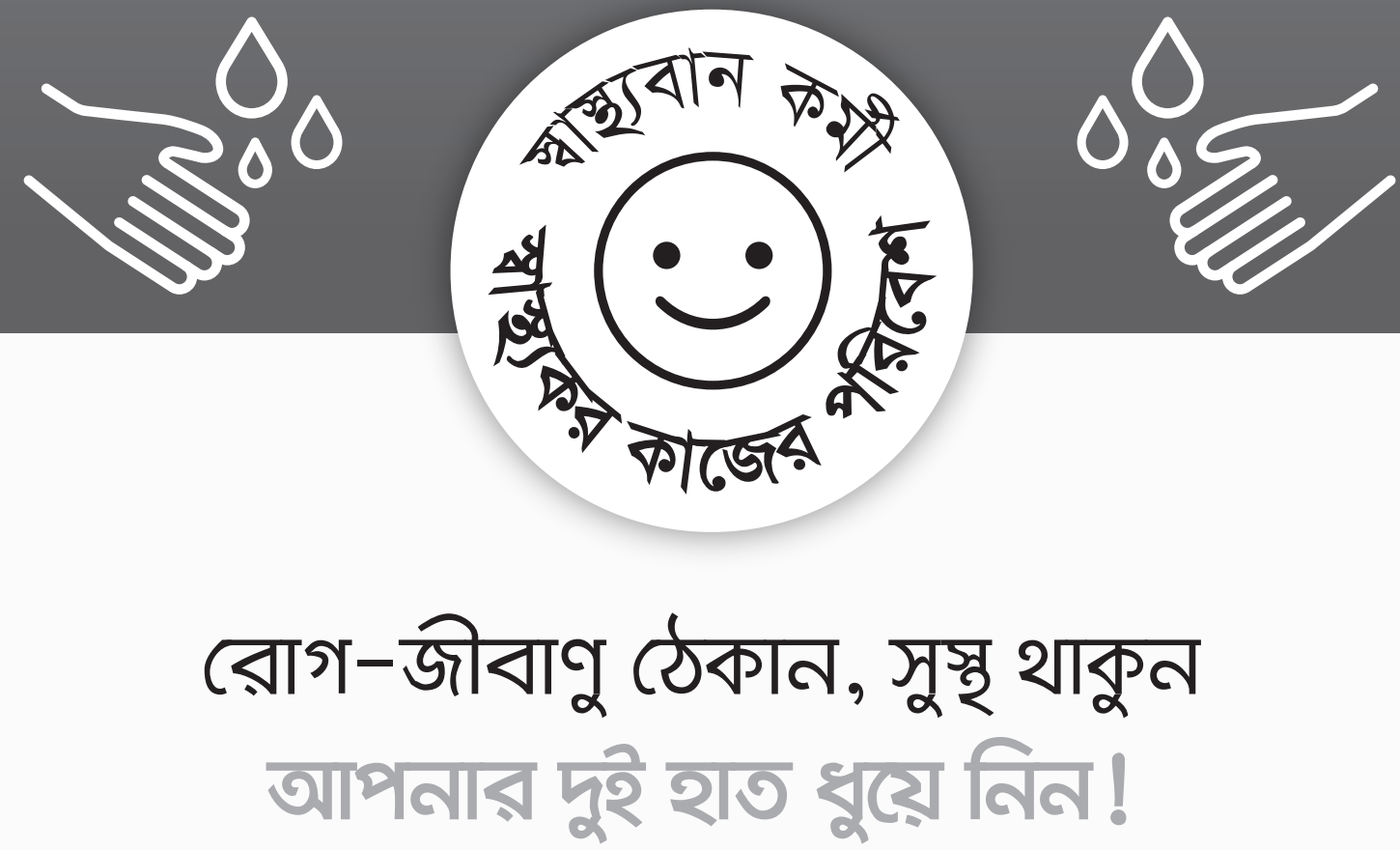

\section{$?$}

কখন আপনার দুই হাত ধোয়া উচিত?

\section{!}

আপনার দুই হাত ধোয়ার

\section{গুরুত্পূর্ণ সময়গুলো হচ্ছ:}

- পায়খানা ব্যবহারের পরে

- খাবার তৈরীর আগে ও পরে

- খাবার খাওয়ার আগে

- কাঁচা মাংস ধরার পরে

- নাক ঝাড়া, কফ-কাশি বা হাঁচির পরে

- পেশাগত সরঞ্জাম ও যন্ত্রপাতি ব্যবহারের পরে

\section{আপনার কর্মন্য় কিংবা বসবা৫র এলাকায় কর্মরত} ম্বাঙ্ছ্য ও পরিবার কল্যাণ সবাদানকরীর সাথে কথা বলুন 

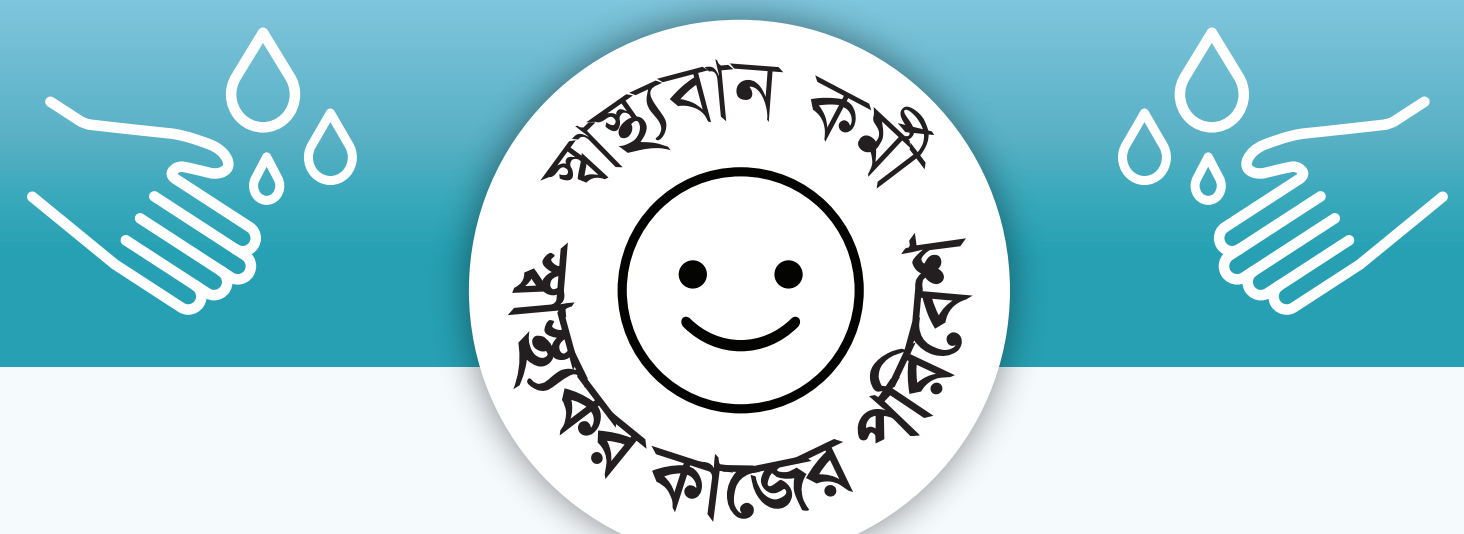

\section{হাত ধোয়ার বিষয়গুলো আপনি জানেন কি ? নিজেকে যাচাই করুন:}

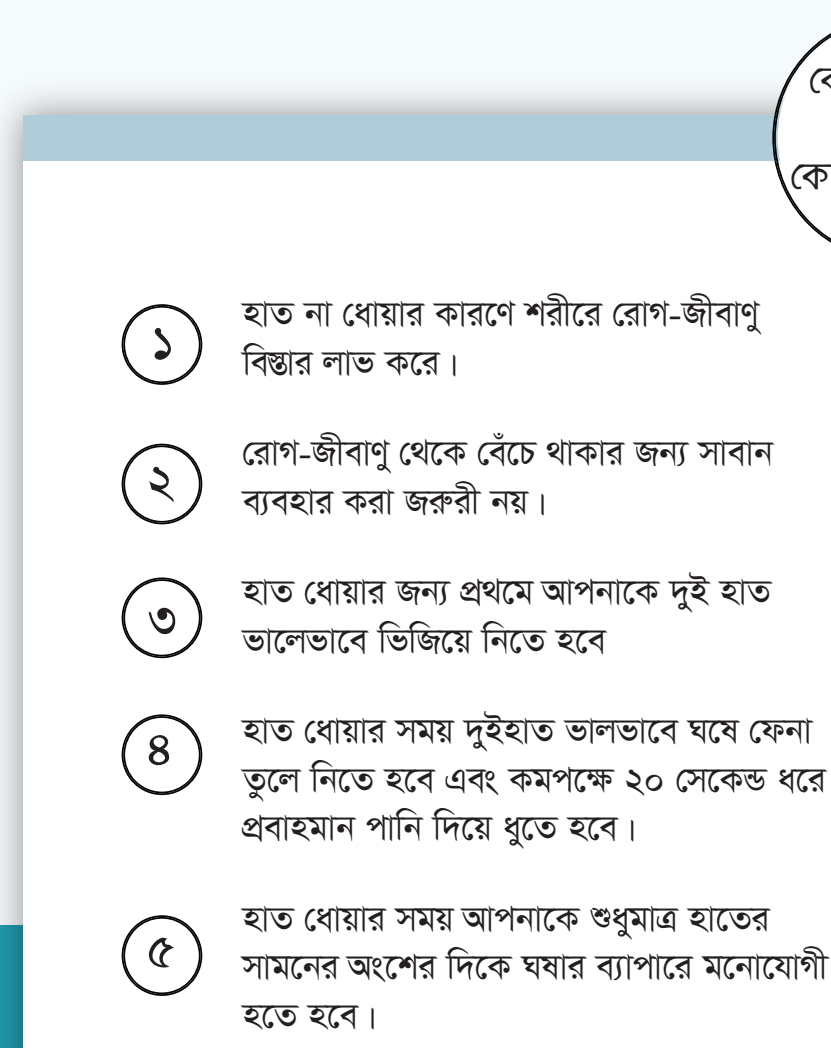
(৬) পায়খানা থেকে বের হওয়ার পর দুই হাত
ধুতে হবে।

(9) খাবার খাওয়ার আগে দুই হাত ধুতে হবে।

(b) সর্দি-কাশি বা হাঁচিতে আক্রান্ত হলে হাত
ধোয়ার প্রয়োজন নেই।

(৯) যদি হাত মোছার জন্য কোন তোয়ালে বা কাপড় না থাকে তাহলে বাতাসে দুই হাত শুকিয়ে নিতে হবে।

\section{উত্তরসমূহ:}

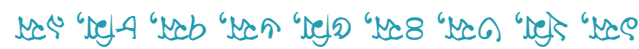

\section{আপনার কর্নছুল কিংবা বসবালর এলাকায় কর্ররত \\ ত্বাজ্য্য ও পরিবার কল্যাণ সবাদানকারীর সাশ্য কথা বলুন}



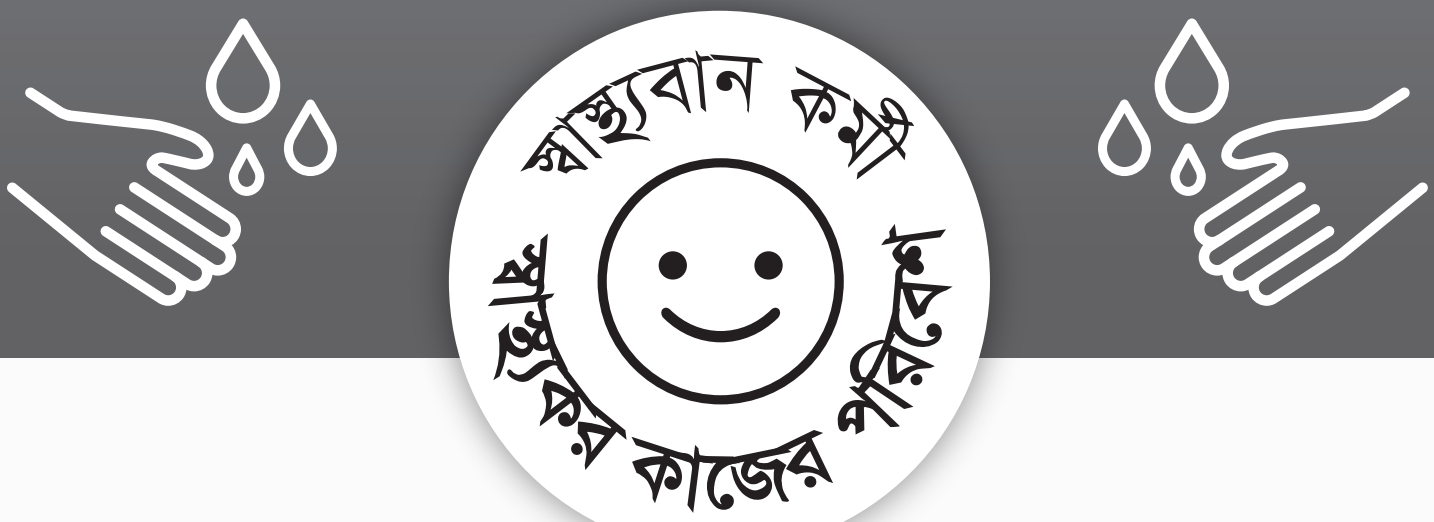

\section{হাত ধোয়ার বিষয়গুলো আপনি জানেন কি ?}

\section{নিজেকে যাভাই করুন:}

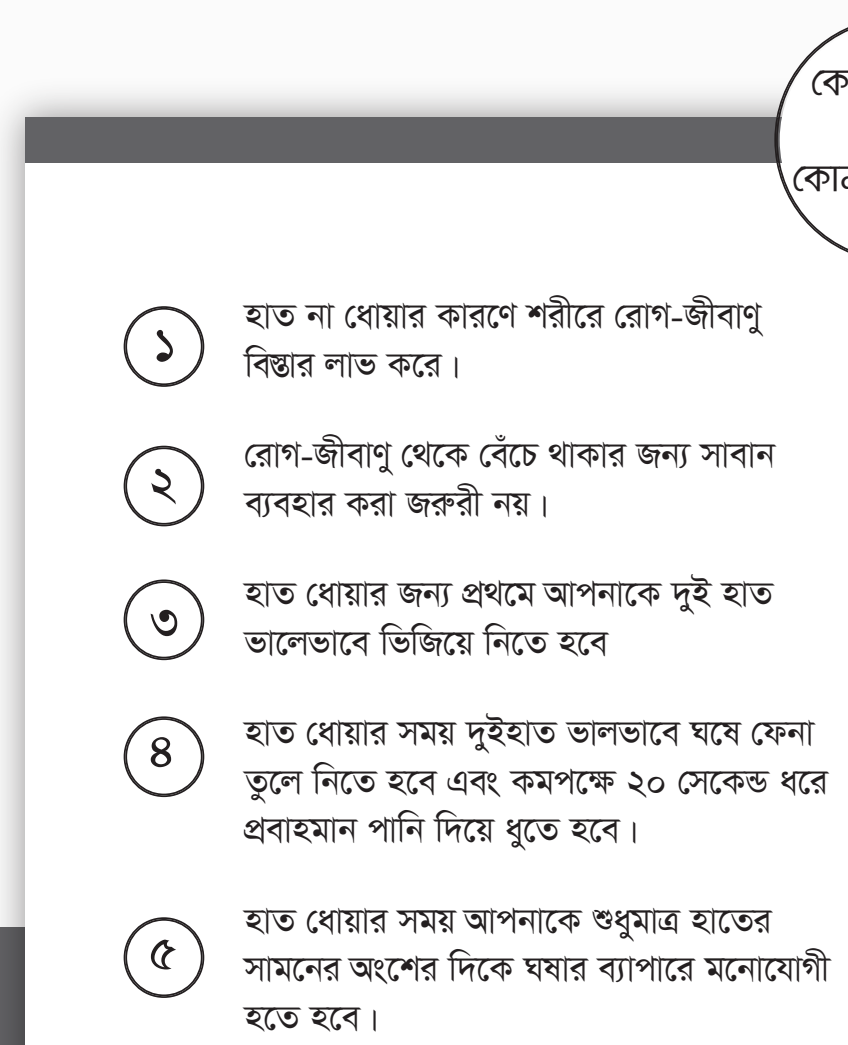

(4) পায়খানা থেকে বের হওয়ার পর দুই হাত ধুতে হবে।

(१) খাবার খাওয়ার আগে দুই হাত ধুতে হবে।

(b) সর্দি-কাশি বা হাঁচিতে আক্রান্ত হনে হাত ধধায়ার প্রয়োজন নেই।

(৯) যদি হাত মোছার জন্য কোন তোয়ালে বা কাপড় না থাকে তাহলে বাতাসে দুই হাত শুকিয়ে নিতে হবে।

\section{ডত্তরসমূহ:}

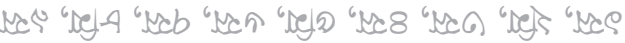

\section{আপনার কর্মন্ইল কিংবা বসবালর এলাকায় কর্মরত স্বান্য্য ও পরিবার কল্যাণ সবাদানকারীর সাথ কথা বলুন}

\section{(18)}



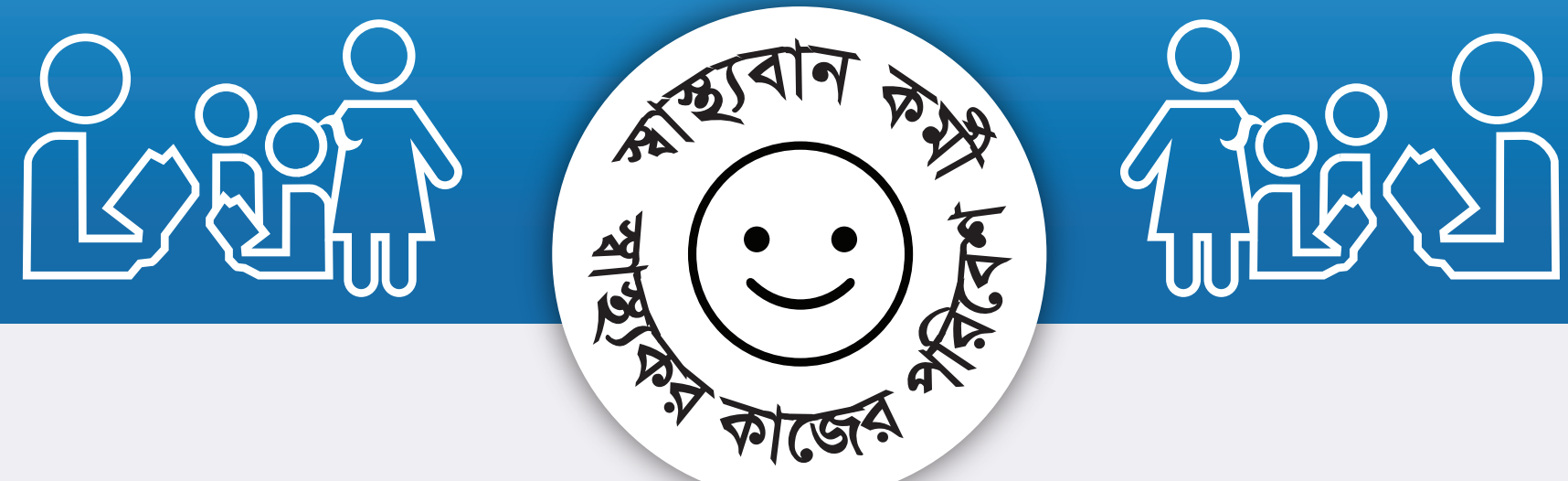

\section{যখন আপনি পরিবারের স্বান্য্যের প্রতি যত্নশীল তখন আপনি নিজের স্বান্য্যুরও যত্ন নেন !}

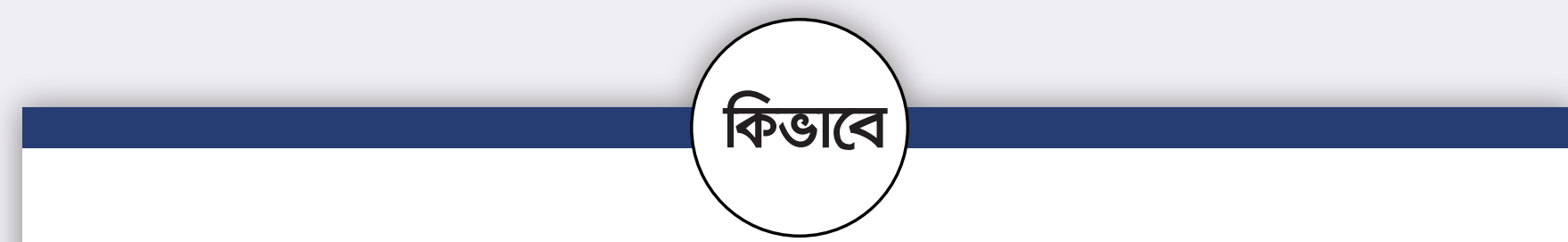

স্ত্ত্রীর সাথে আলোচনা করে সন্তান ধারণ ও দুই সন্তানের মাঝে বিরতির পরিকল্পনা করুন। আপনি এবং আপনার শ্র্রী মিলে সিদ্ধান্ত নিন যে আপনারা কখন ও কয়টি সন্তান নেবেন। পুনরায় গর্ভধারণ করতে চাইনে আপনার সর্বশেষ সন্তানের বয়স কমপক্ষে ২ বছর হওয়া পর্যন্ত অপেক্ষা করুন। বয়সের এই ব্যবধানটি মায়ের নিরাপদদভাবে সুমৃ শিশ্ঠ জন্দদানের সম্ভাবনাকে উল্লেখযোগ্যভাবে বৃদ্ধি করতে সহায়তা করে। একটি সুমৃ সন্তান মানে তার জীবনের আপনার পরিবারের দেখাশোনায় অংশগ্রহণ করুন

সন্তানদের স্কুলে নিয়ে যাওয়া ও নিয়ে আসা এবং তাদের স্বাম্থ্যের যত্ন নেওয়া আপনার একান্ত দায়িত্ব ও কর্তব্য। তাদের সাথে খেলাধুলা করা এবং সময় কাটানো একটি গুরুত্বপূর্ণ কাজ। পাশাপাশি পরিবারের অর্থনৈতিক চাহিদা পূরণ করা আপনার গুরুত্বপূর্ণ দায়িত্ব।

ও স্কুলের ভালো সূচনা হওয়া।

আপনার সন্তানদের স্বাস্থ্য কেন্দ্রে নিয়ে যান অসুন্তা থেকে আপনার সন্তানদের রক্ষা করতে আপনি খুবই গুরুত্বপূর্ণ ভূমিকা পালন করতে পারেন। আপনার সন্তানদেরকে নিয়মিত টিকা দিয়ে রোগের ঝুঁকিমুক্ত রাখা একটি গুরুত্বপূর্ণ কাজ যা বাবা হিসেবে আপনি করতে পারেন।
পরিবার সুন্থ তো আপন্নিও সুন্ছ!

আন্তরিক অভিভাবক হওয়া

আপনার জন্য মঙ্গলজনক

\section{আপনার কর্মন্ইল কিংবা বসবালার এলাকায় কর্মরত} স্বাষ্ছ্য ও পরিবার কল্যাণ সেবাদানকারীর সাথ কথা বলুন 

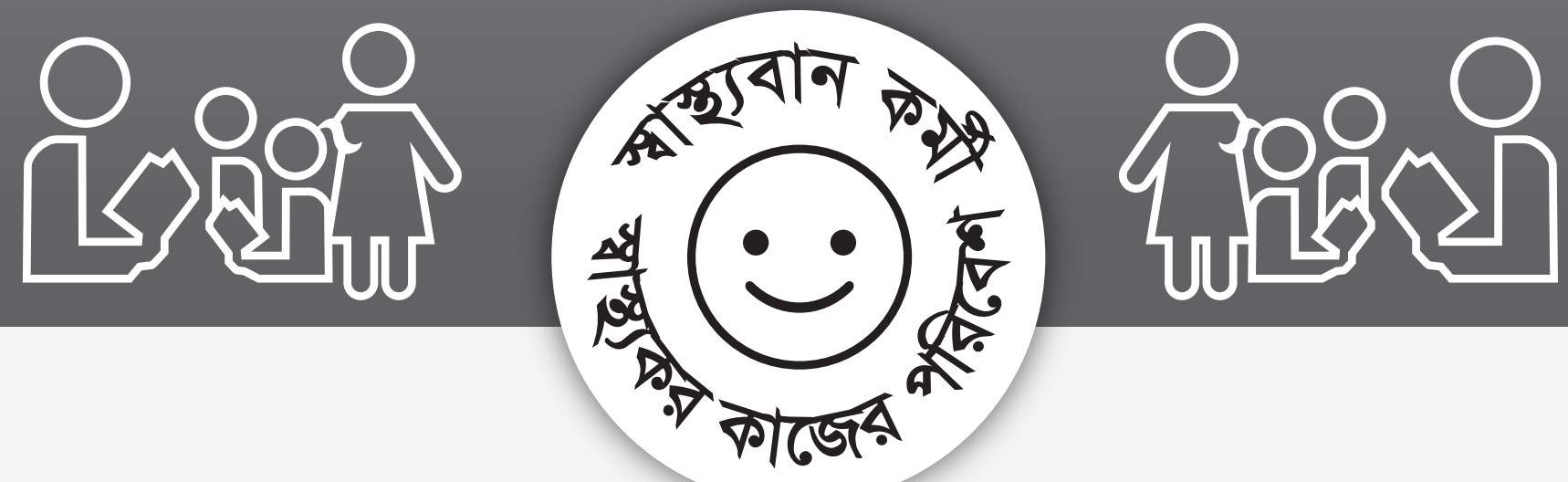

\section{যখন আপনি পরিবারের স্বান্য্যের প্রতি যত্নশীল তখন আপনি নিজের স্বান্য্যুরও যত্ন নেন !}

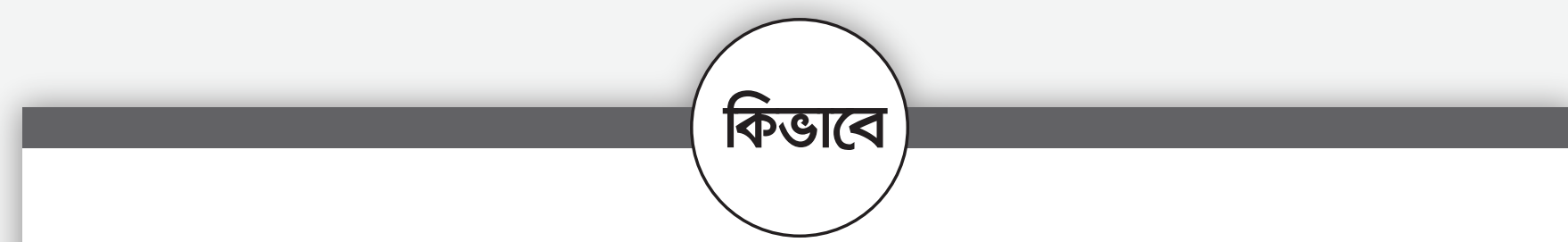

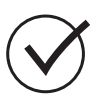
স্ত্রীর সাথে আলোচনা করে সন্তান ধারণ ও দুই সন্তানের মাঝে বিরতির পরিকল্পনা করুন। আপনি এবং আপনার শ্র্রী মিলে সিদ্ধান্ত নিন যে আপনারা কখন ও কয়টি সন্তান নেবেন। পুনরায় গর্ভধারণ করতে চাইনে আপনার সর্বশেষ সন্তানের বয়স কমপক্ষে ২ বছর হওয়া পর্যন্ত অপেক্ষা করুন। বয়সের এই ব্যবধানটি মায়ের নিরাপদভাবে সুম্থ শিশ্ড জন্মদানের সম্ভাবনাকে উল্লেখযোগ্যভাবে বৃদ্ধি করতে সহায়তা করে। একটি সুমৃ সন্তান মানে তার জীবনের ও স্কুলের ভালো সূচনা হওয়া।

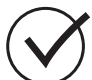
আপনার সন্তানদের স্বাস্থ্য কেন্দ্রে নিয়ে যান অসুসৃতা থেকে আপনার সন্তানদের রক্ষা করতে আপনি খুবই গুরুত্বপূর্ণ ভূমিকা পালন করতে পারেন। আপনার সন্তানদেরকে নিয়মিত টিকা দিয়ে রোগের ঝুঁকিমুক্ত রাখা একটি গুরুত্বপূর্ণ কাজ যা বাবা হিসেবে আপনি করতে পারেন।
আপনার পরিবারের দেখাশোনায় অংশগ্রহণ

করুন

সন্তানদের স্কুনে নিয়ে যাওয়া ও নিয়ে আসা এবং তাদের স্বাম্থ্যের যত্ন নেওয়া আপনার একান্ত দায়িত্ব ও কর্তব্য। তাদের সাথে খেলাধুলা করা এবং সময় কাটানো একটি গুরুত্বপূর্ণ কাজ। পাশাপাশি পরিবারের অর্থনৈতিক চাহিদা পূরণ করা আপনার গুরুত্বপূর্ণ দায়িত্ব।

\section{পরিবার সুন্থ তো আপনিও সুন্ছ!}

আন্তরিক অভিভাবক হওয়া আপনার জন্য মঙ্গলজনক

\section{আপনার কর্মন্ইল কিংবা বসবালর এলাকায় কর্মরত}

ঘ্যান্য্য ও পরিবার কল্যাণ লেবাদানকারীর সাথ কথা বলুন 


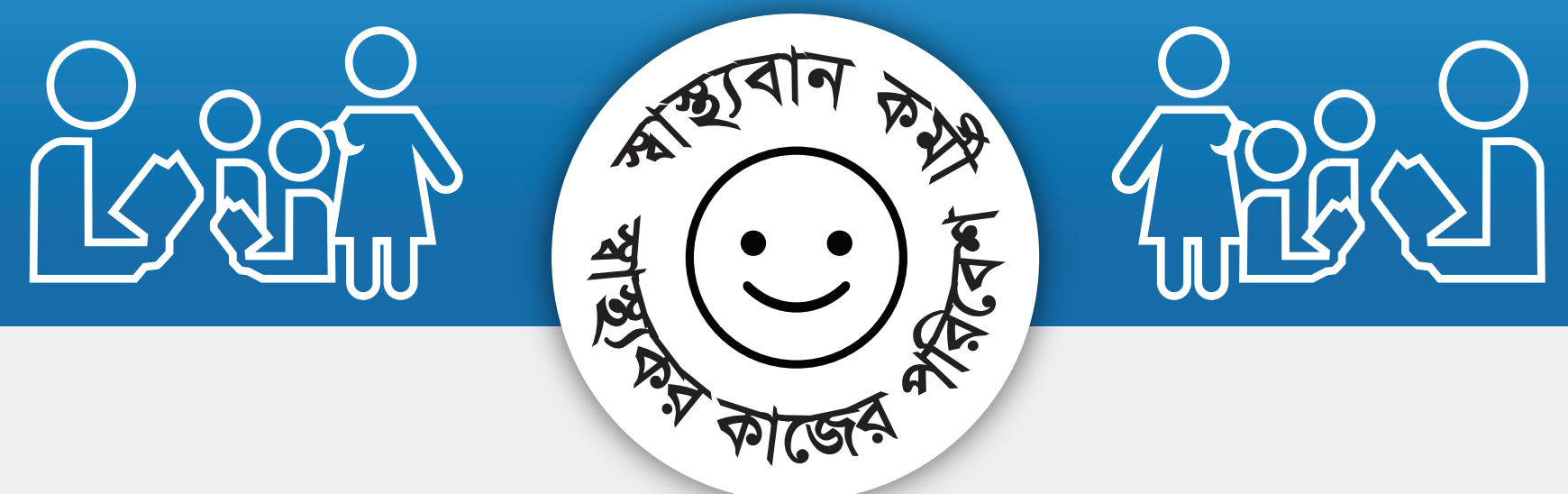

\section{বাবাও যত্নবান হতে পারেন \\ তাদের নিজ নিজ পরিবারের সদস্যদের স্বাস্থ্যের!}
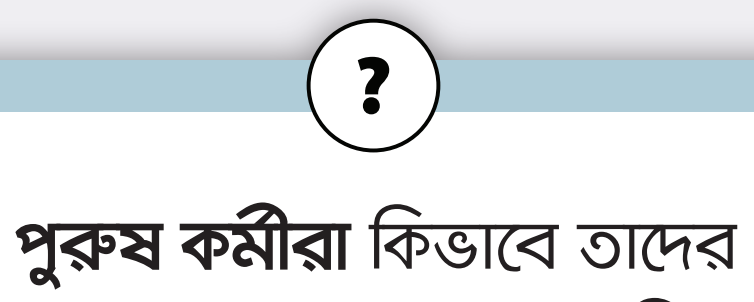

পরিবারের সদস্যদের স্বাস্থ্যের যত্ন নিতে পারেন?

\section{!}

যত্নের কাজগুলো ভাগ করে নিন

- আপনার সন্তানদের ক্কুলে পৌঁছে দিন এবং নিয়ে আসুন

- আপনার সন্তানদের সাথে খেলাধূলা করুন এবং তাদের সাথে সময় কাটান

- আপনার সন্তানদের স্বাস্থ্যকেন্দ্রে নিয়ে যান

- আপনার সন্তানদের টিকাকেন্দ্রে নিয়ে যান

- আলাপ-আলোচনার মাধ্যমে পারিবারিক বিরোধগুনো মিটিয়ে ফেলুন

আপনার কর্মইল কিংবা বসবা৫র এলাকায় কর্মরত স্বাম্ছ্য ও পরিবার কল্যাণ লেবাদানকারীর সাথে কথা বলুন 


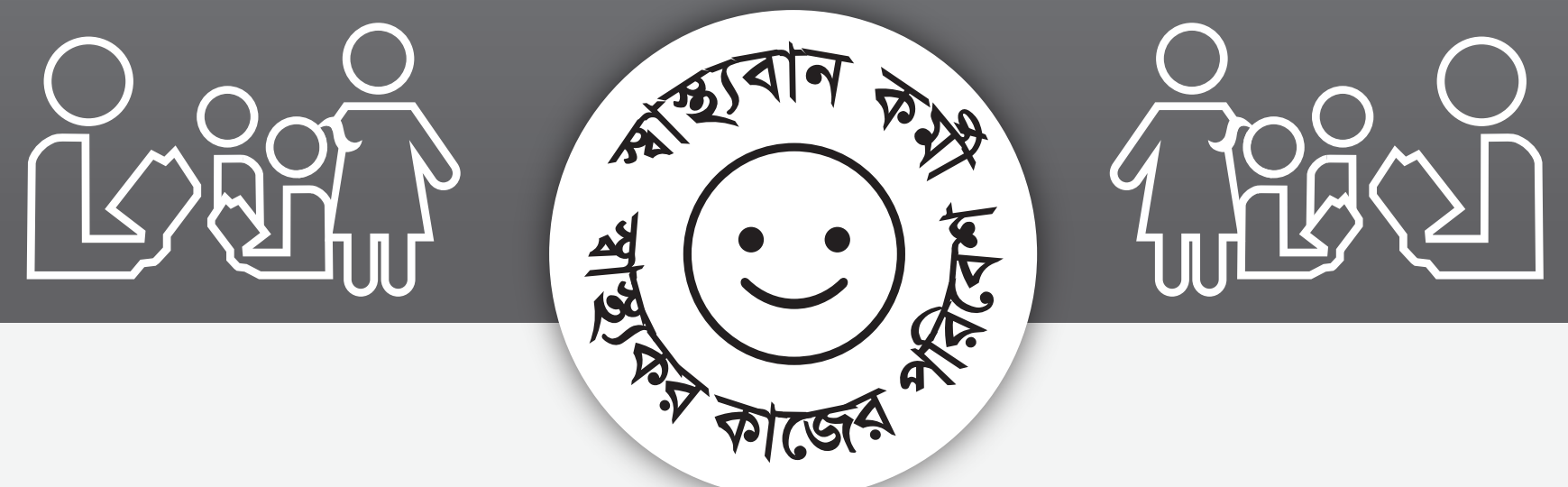

\section{বাবাও যত্নবান হতে পারেন \\ তাদের নিজ নিজ পরিবারের সদস্যদের স্বাস্থ্যের!}

\section{$?$}

পুরুষ কনীরা কিভাবে তাদর

পরিবারের সদস্যদের স্বাম্য্যের যত্ন নিতে পারেন?

\section{!}

যত্নের কাজগুলো ভাগ করে নিন

- আপনার সন্তানদের ক্কুলে পৌঁছে দিন এবং নিয়ে আসুন

- আপনার সন্তানদের সাথে খেলাধূলা করুন এবং তাদের সাথে সময় কাটান

- আপনার সন্তানদের স্বাস্থ্যকেন্দ্রে নিয়ে যান

- আপনার সন্তানদের টিকাকেন্দ্রে নিয়ে যান

- আলাপ-আলোচনার মাধ্যমে পারিবারিক বিরোধগুনো মিটিয়ে ফেলুন

আপনার কর্মইল কিংবা বসবাঙর এলাকায় কর্মরত

স্যান্ছ্য ও পরিবার কল্যাণ সবাদানকরীর সাথ কথা বলুন 


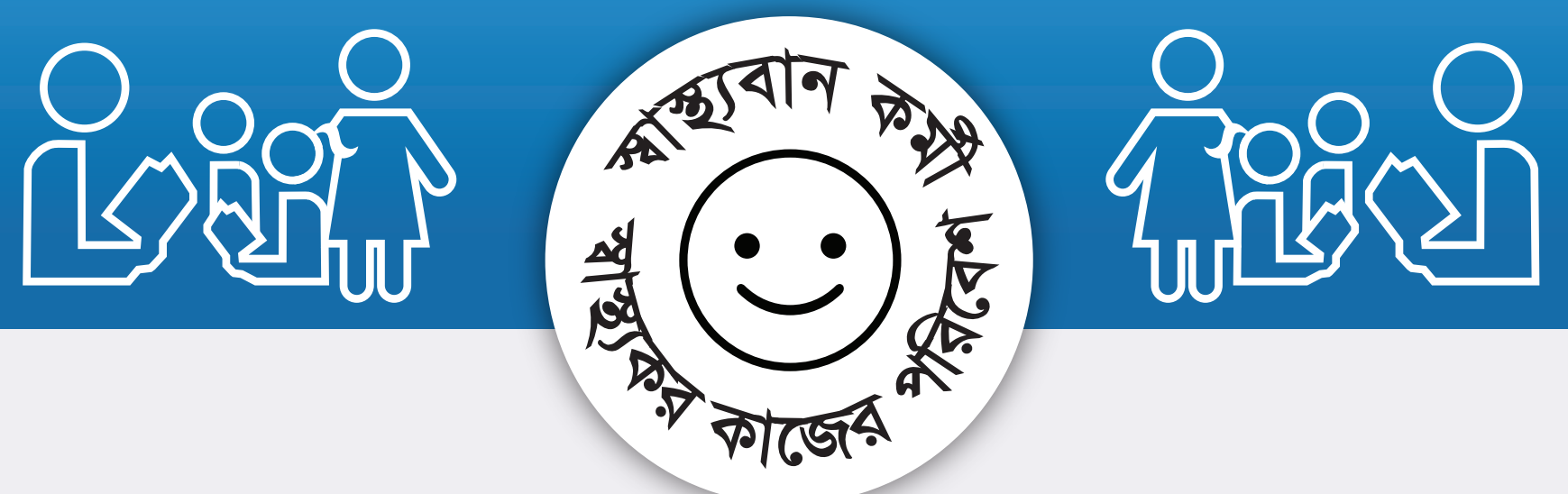

\section{বাবাও যত্নবান হতে পারেন \\ তাদের নিজ নিজ পরিবারের সদস্যদের স্বাস্থ্যের!}

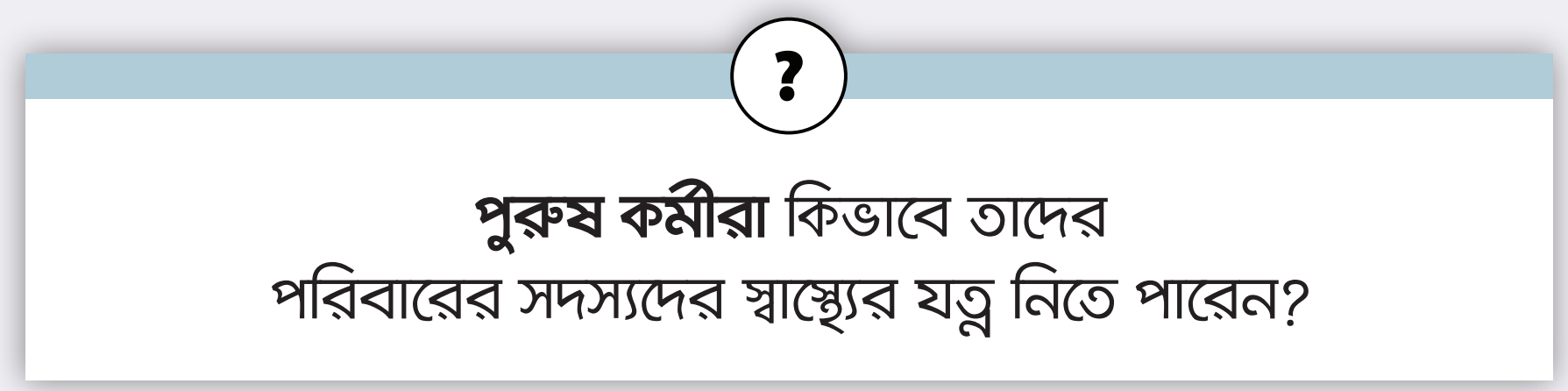

\section{!}

\section{শুরু থেকেই সম্পৃক্ত হোন}

- আপনার শ্ত্রীর সাথে পরিবারের আকার সীমিত রাখার বিষয় নিয়ে পরিকল্পনা করুন

- স্বাস্থ্যবান মা, শিশু ও পরিবারের জন্য পরপর দুইবার গর্ভধারণের মধ্যে কমপক্ষে দুই বছরের বিরতি দিন

- গর্ভকালীন সময়ে ত্ত্রীকে পারিবারিক ও অন্যান্য কাজে সহায়তা করুন

- আপনার গর্ভবতী ত্ত্রীকে কমপক্ষে চারবার স্বাস্থ্যকেন্দ্রে নিয়ে যান

- গর্ভকালীন সময়ে আপনার স্ত্রীর পুষ্টির বিষয়ে সতর্ক থাকুন

আপনার কর্নন্ইল কিংবা বসবা৫র এলাকায় কর্মরত স্বাম্ছ্য ও পরিবার কল্যাণ সবাদানকরীর সাথ কথা বলুন

(ㅇ) 


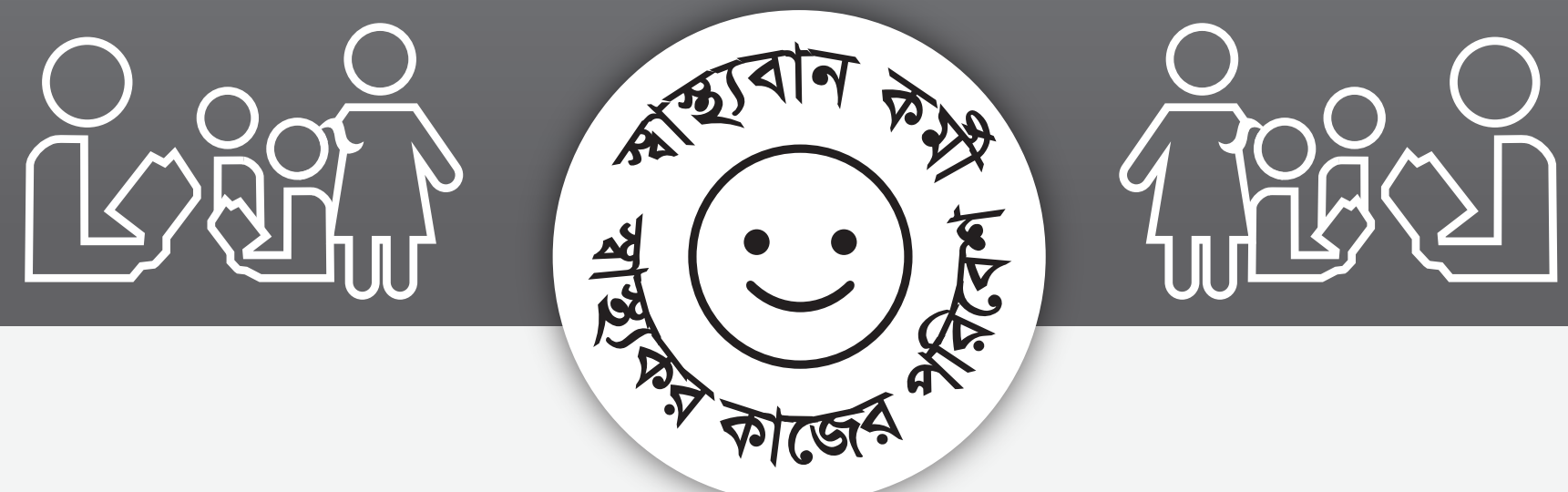

\section{বাবাও যত্নবান হতে পারেন \\ তাদের নিজ নিজ পরিবারের সদস্যদের স্বাস্থ্যের!}

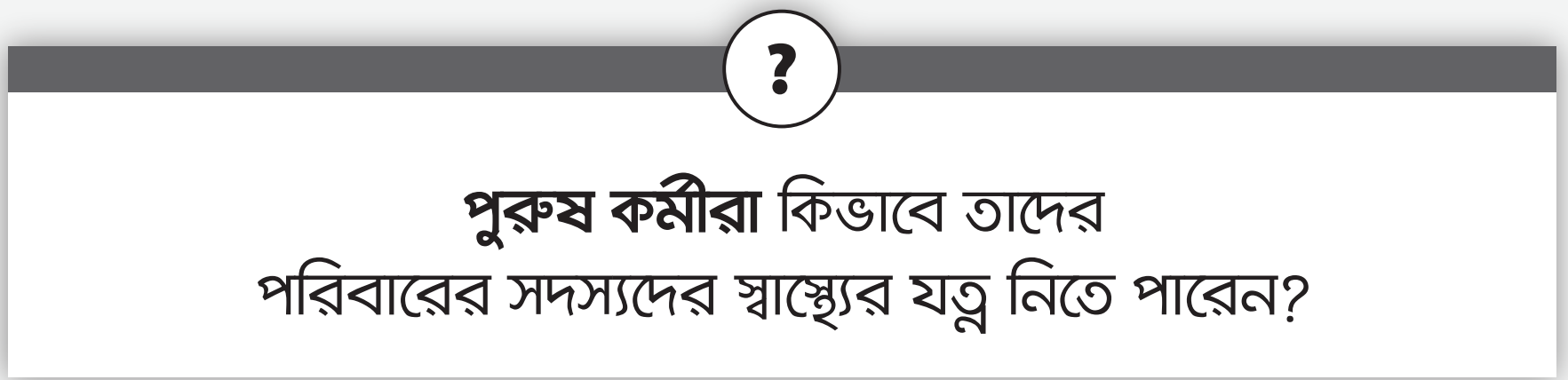

\section{!}

\section{শুরু থেকেই সম্পৃক্ত হোন}

- আপনার শ্ত্রীর সাথে পরিবারের আকার সীমিত রাখার বিষয় নিয়ে পরিকল্পনা করুন

- স্বাস্য্যবান মা, শিশু ও পরিবারের জন্য পরপর দুইবার গর্ভধারণের মধ্যে কমপক্ষে দুই বছরের বিরতি দিন

- গর্ভকালীন সময়ে ত্ত্রীকে পারিবারিক ও অন্যান্য কাজে সহায়তা করুন

- আপনার গর্ভবতী ত্ত্রীকে কমপক্ষে চারবার স্বাস্থ্যকেন্দ্রে নিয়ে যান

- গর্ভকালীন সময়ে আপনার শ্ত্রীর পুষ্টির বিষয়ে সতর্ক থাকুন

আপনার কর্মন্ইল কিংবা বসবা৫র এলাকায় কর্মরত স্বাম্ছ্য ও পরিবার কল্যাণ সবাদানকরীর সাথ কথা বলুন 